\title{
Supporting Material:
}

\section{Arene C-H Functionalization by P-Block Metal TI(III) Occurs at the Borderline of $\mathrm{C}-\mathrm{H}$ Activation and Electron Transfer}

Clinton R. King, ${ }^{[a]}$ Samantha J. Gustafson, ${ }^{[a]}$ Benjamin R. Black, ${ }^{[a]}$ Steven K. Butler, ${ }^{[a]}$ Michael M. Konnick, ${ }^{[b]}$ Roy A. Periana, ${ }^{[c]}$ Brian M. Hashiguchi, ${ }^{[b]}$ and Daniel H. Ess ${ }^{\star[a]}$ ${ }^{[a] D e p a r t m e n t}$ of Chemistry and Biochemistry, Brigham Young University, Provo, UT 84602, (USA)

[b]Hyconix, Inc. 4575 Weaver Pkwy, Warrenville, IL 60555 (USA)

[c]Department of Chemistry, The Scripps Research Institute, Jupiter, FL 33458 (USA)

*dhe@chem.byu.edu

\section{Table of Contents}

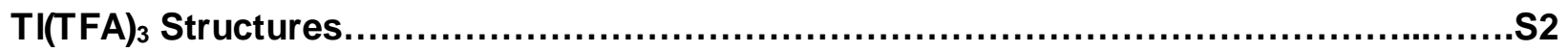

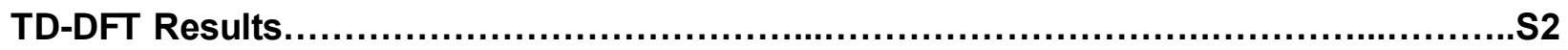

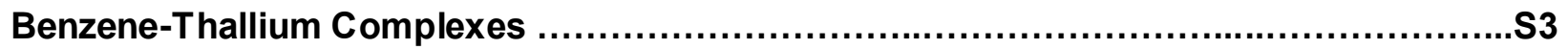

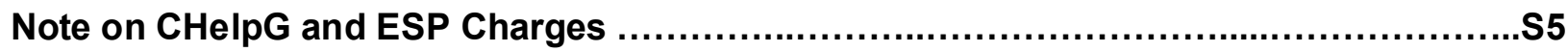

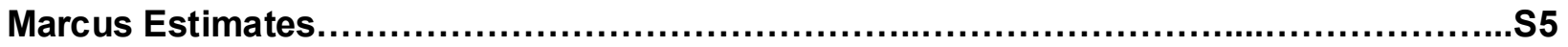

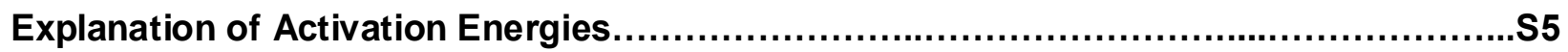

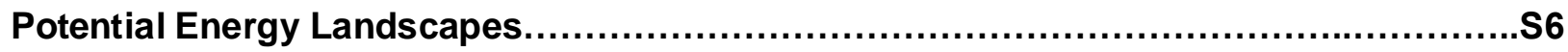

Arene Coordination versus Activation Barriers..................................................8

SMD Solvated (U)M06/Def2-TZVPPD//(U)M06/6-31+G(d,p)[LANL2DZdp] XYZ Coordinates

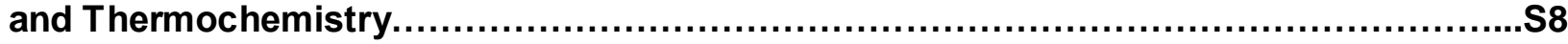




\section{TI(TFA) $)_{3}$ Structures}

Structure $1 \mathrm{~A}$ shown in figure $\mathrm{S} 1$ below is the $\mathrm{TI}(\mathrm{OAc})_{3} \mathrm{x}$-ray structure. ${ }^{1}$ Change of acetate ligands to TFA ligands and optimization resulted in structure 1B. We also located low-energy structures 1C and 1D. The lowest energy structures are 1C and 1D that both have $C_{s}$ symmetry. The enthalpies of these structures are within about $1.1 \mathrm{kcal} / \mathrm{mol}$ of each other. The energy of $1 \mathbf{D}$ was adopted as the ground state energy.

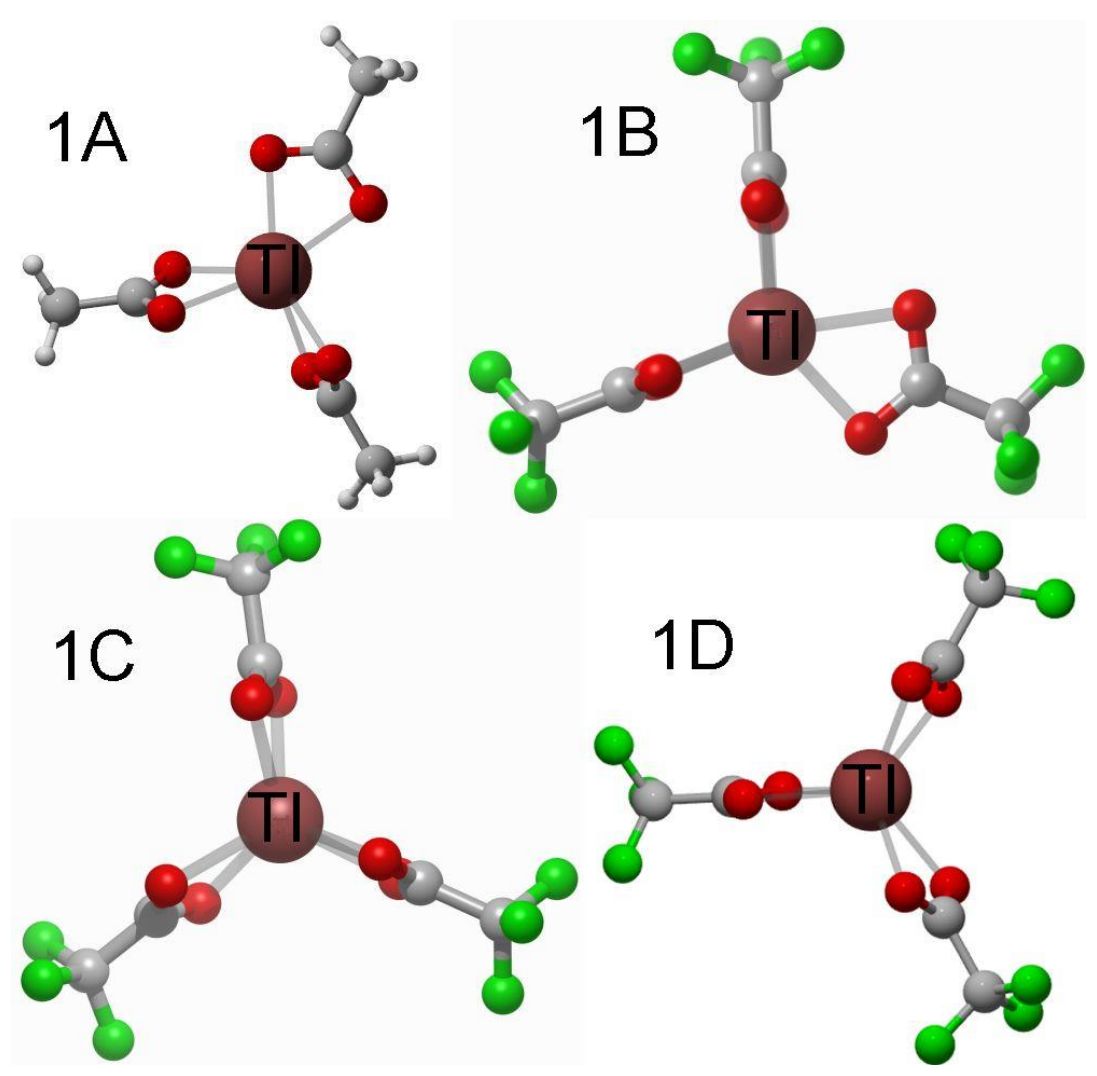

Figure S1. Top: $\mathrm{TI}(\mathrm{OAC})_{3} \mathrm{x}$-ray structure and the corresponding $\mathrm{TI}(\mathrm{TFA})_{3}$ structure optimized in implicit trifluoroacetic acid solvent. Bottom: Low-energy TI(TFA) ${ }_{3}$ structures.

\section{TD-DFT Results}

Below lists the major M06 TDDFT excitations for $\pi$-complexes between benzene with TI(TFA $)_{3}$, $\left[\mathrm{TI}(\mathrm{TFA})_{2}\right]^{+}$, and $\left[\mathrm{TI}(\mathrm{TFA})_{2}(\mathrm{TFAH})\right]^{+}$(Figure S2). All excitations are qualitatively consistent with the spectra reported by Kochi, and therefore, this does not provide a way to distinguish between neutral and cationic species. ${ }^{2}$ 
Table S1. TDDFT results.

\begin{tabular}{ccc} 
Structure & Excitation Wavelength & Oscillator Strength \\
\hline$\left[\mathrm{TI}(\mathrm{TFA})_{3}\left(\mathrm{C}_{6} \mathrm{H}_{6}\right)\right]$ & 329 & 0.24 \\
{$\left[\mathrm{TI}(\mathrm{TFA})_{2}\left(\mathrm{C}_{6} \mathrm{H}_{6}\right)\right]^{+}$} & 345 & 0.30 \\
{$\left[\mathrm{TI}(\mathrm{TFA})_{2}(\mathrm{TFAH})\left(\mathrm{C}_{6} \mathrm{H}_{6}\right)\right]^{+}$} & 336 & 0.32
\end{tabular}

Figure S2 shows the Kohn-Sham orbitals that give rise to the TDDFT excitation at $329 \mathrm{~nm}$ for the TI(TFA) $)_{3}$-benzene $\pi$ complex. These orbitals show the charge-transfer character from the arene to the TI metal center. This wavelength is close to the wavelength reported by Kochi and Lau for charge transfer between benzene and TI(III) in trifluoroacetic acid solvent.
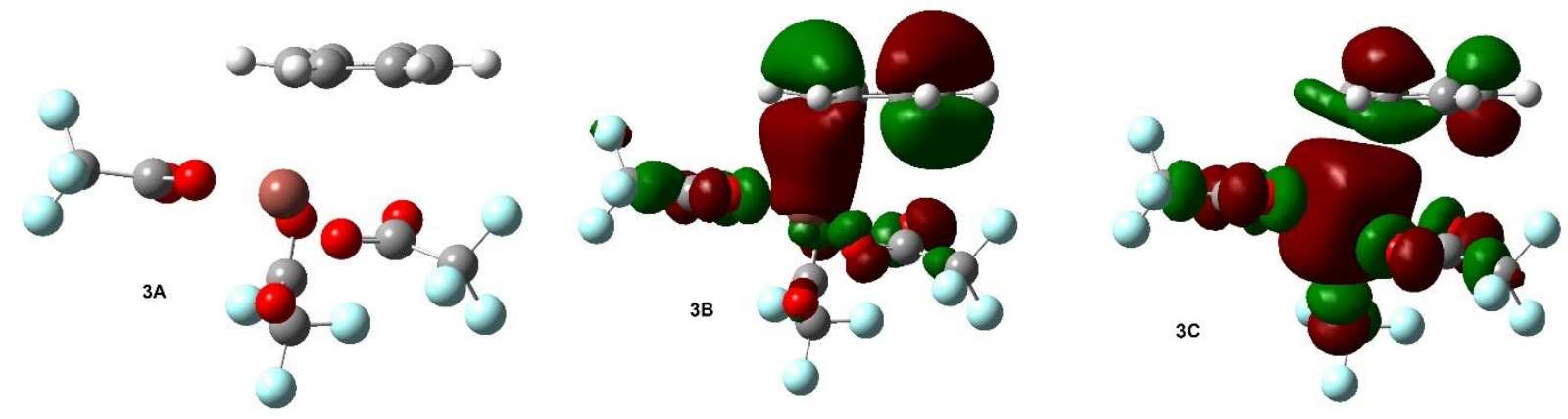

Figure S2. Relevant Kohn-Sham M06 orbitals for the $329 \mathrm{~nm}$ excitation.

\section{Benzene-Thallium Complexes}

We examined the coordination modes of benzene to TI(TFA)3 by starting with structures in which benzene was constrained in various coordination modes. All of the internal coordinates of these structures were allowed to relax except for C-TI distances, which were fixed to maintain the proper coordination mode. When all modes were allowed to relax, the preferred binding modes were found to be $\eta^{1}$ or $\eta^{2}$ (see Table S2 and Figure S3). 


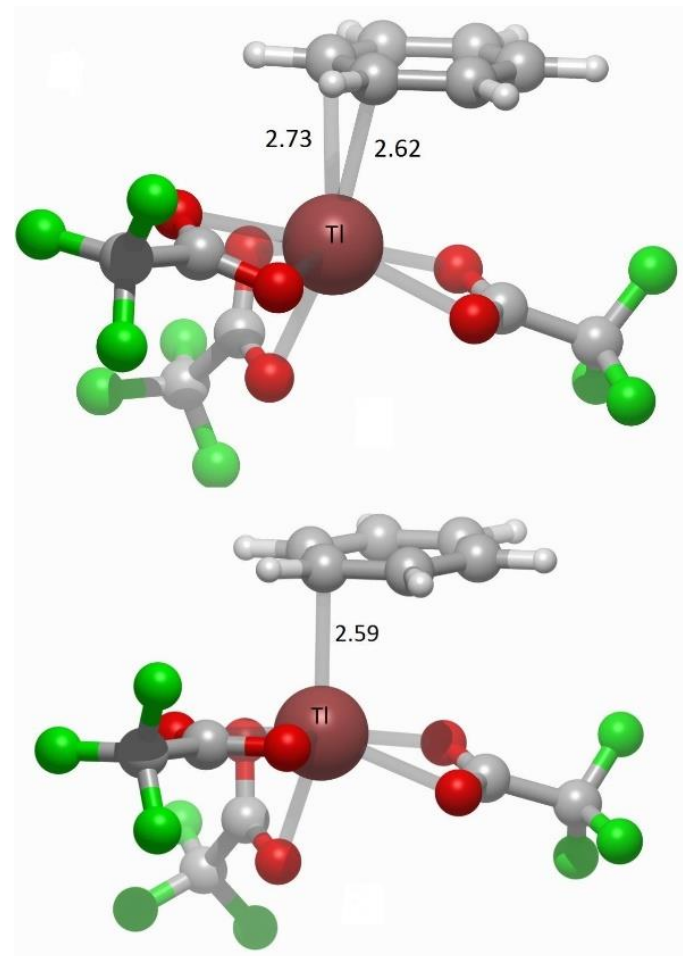

Figure S3. Representative $\pi$-complex structures of benzene and TI(TFA $)_{3}$.

Table S2. Relative energies of various $\pi$-complexes of benzene and TI(TFA $)_{3}$.

\begin{tabular}{|c|c|c|c|c|}
\hline $\begin{array}{l}\text { Constrained } \\
\text { coordination } \\
\text { mode }\end{array}$ & $\begin{array}{l}\text { constrained } \\
\text { C-TI } \\
\text { distance (Å) }\end{array}$ & $\begin{array}{l}\text { Relative }{ }^{\mathrm{a}} \text { electronic } \\
\quad \text { energy }^{\mathrm{b}} \text { of } \\
\text { constrained } \\
\text { structure (kcal/mol) }\end{array}$ & $\begin{array}{l}\text { Relative }^{\mathrm{a}} \\
\text { electronic energy }^{\mathrm{b}} \\
\text { of relaxed }^{\mathrm{c}} \\
\text { structure } \\
\text { (kcal } / \mathrm{mol})\end{array}$ & $\begin{array}{l}\Delta H(\Delta G)^{\mathrm{a}, \mathrm{d}} \text { of } \\
\quad \text { relaxed } \\
\text { structure } \\
(\mathrm{kcal} / \mathrm{mol})\end{array}$ \\
\hline$\eta^{1}$ & 2.4 & -14.2 & -15.5 & $-12.6(0.2)$ \\
\hline$\eta^{2}$ & 2.5 & -13.5 & -15.4 & $-12.7(0.2)$ \\
\hline$\eta^{3}$ & 2.6 & -1.9 & -15.5 & $-12.5(2.1)$ \\
\hline$\eta^{4}$ & 2.7 & 0.5 & -15.7 & $-12.9(1.4)$ \\
\hline$\eta^{5}$ & 2.8 & -2.4 & -15.6 & $-12.3(2.5)$ \\
\hline$\eta^{6}$ & 2.9 & -4.8 & -15.8 & $-13.2(-0.4)$ \\
\hline \multicolumn{5}{|c|}{$\begin{array}{l}\text { a Relative to separated benzene and TI(TFA) } \\
\text { b. } \\
\text { Electronic energy from M06/6-31+G(d,p)[LANL2DZdp] SMD solvent calculation. } \\
{ }^{c} \text { All structures relaxed to } \eta^{1} \text { or } \eta^{2} \text { complexes. } \\
\text { d Free energies and enthalpies from M06/def2-TZVPPD/M06/6-31+G(d,p)[LANL2DZdp] with } \\
\text { SMD solvent. }\end{array}$} \\
\hline
\end{tabular}




\section{Note on CHelpG and ESP Charges}

A value of $\sim 0.35$ e of charge transfer from benzene to TIIII in (TFA) ${ }_{3} \mathrm{TI}^{\prime \prime \prime}\left(\eta^{1}-\mathrm{C}_{6} \mathrm{H}_{6}\right)$ was based on $\mathrm{CHelpG}$ and ESP atomic charges. This value is the result of summing atomic charges on all atoms in the $\mathrm{TI}(\mathrm{TFA})_{3}$ fragment of the complex and summing the atomic charges on the benzene fragment in the complex. The atomic charges used are determined by fitting partial atomic charges to a molecular electrostatic potential. The CHelpG and ESP algorithms select points in a grid surrounding the Van der Waals surface. ${ }^{3}$

\section{Marcus Estimates}

Marcus theory estimates the kinetic barrier for outer-sphere electron transfer (ET) by using reorganization energy, $\lambda$, and free energy change:

$$
\Delta G^{\ddagger}=\frac{\left(\lambda+\Delta G^{o}\right)^{2}}{4 \lambda}
$$

The internal structural reorganization energy $\left(\lambda_{i}\right)$ is given by ${ }^{4,5}$ :

$$
\lambda_{i}=E_{R}\left(R_{P}\right)-E_{R}\left(R_{R}\right)+E_{P}\left(R_{R}\right)-E_{P}\left(R_{P}\right)
$$

The solvent/outersphere reorganization energy $\left(\lambda_{o}\right)$ is given by:

$$
\lambda_{o}=(\Delta e)^{2}\left(\frac{1}{2 r_{A}}+\frac{1}{2 r_{B}}-\frac{1}{r_{A B}}\right)\left(\frac{1}{\varepsilon_{\infty}}-\frac{1}{\varepsilon_{o}}\right)
$$

For trifluoroacetic acid, $\varepsilon_{\infty}=1.285$ and $\varepsilon_{0}=8.42$.

For ET between $\mathrm{TI}(\mathrm{TFA})_{3}$ and arenes, the Marcus estimates reported in the main text ignore solvent reorganization energy. For benzene this estimate is $\Delta G^{\ddagger}=36.8 \mathrm{kcal} / \mathrm{mol}$. For pentamethylbenzene, $\Delta G^{\ddagger}=13.5 \mathrm{kcal} / \mathrm{mol}$. Estimate of the solvent reorganization energy is dependent on the radii. For intermolecular distances of less than $10 \AA$, including the solvent reorganization energy increases the overall $\Delta G^{\ddagger}$ by less than $5 \mathrm{kcal} / \mathrm{mol}$.

\section{Explanation of Activation Energies}

Relative arene thallation rates (Figure 2 in the manuscript) were converted into free energy differences $\left(\Delta \Delta G^{\ddagger}\right)$ using standard transition state theory with cancellation of pre-exponential factors, i.e.: $k_{r}=k_{1} / k_{2}=e^{-\left(\Delta G_{1} \ddagger-\Delta G_{2} \ddagger\right) / R T}$. For regioselectivity data reported by McKillop ${ }^{6}$ and $\mathrm{Olah}^{7}$, free energy differences were derived from product ratios by assuming that the reported product ratio is equal to a ratio of rate constants. 


\section{Potential Energy Landscapes}

Figure S4 shows the energy landscape where the TI-C bond has been scanned from the distance in the $\pi$ complex $(\sim 2.6 \AA)$ to the distance in the thallation product $(\sim 2.2 \AA)$, and the TI-C-H angle has been scanned from that in the $\pi$ complex $\left(\sim 90^{\circ}\right)$ to an approximately tetrahedral angle $\left(\sim 110^{\circ}\right)$ that might be expected in a Wheland intermediate. Importantly, all Wheland-type structures are much higher in energy than the $\eta^{1} \pi$-complex structure. Additionally, scans of the same coordinates as shown in Scheme 3 were performed using energies from the $\omega B$ B7xd, M06L, and B3LYP functionals, and the M06 functional with an IEF-PCM solvent model. PES's from these scans are qualitatively identical to the one shown in Scheme 3.

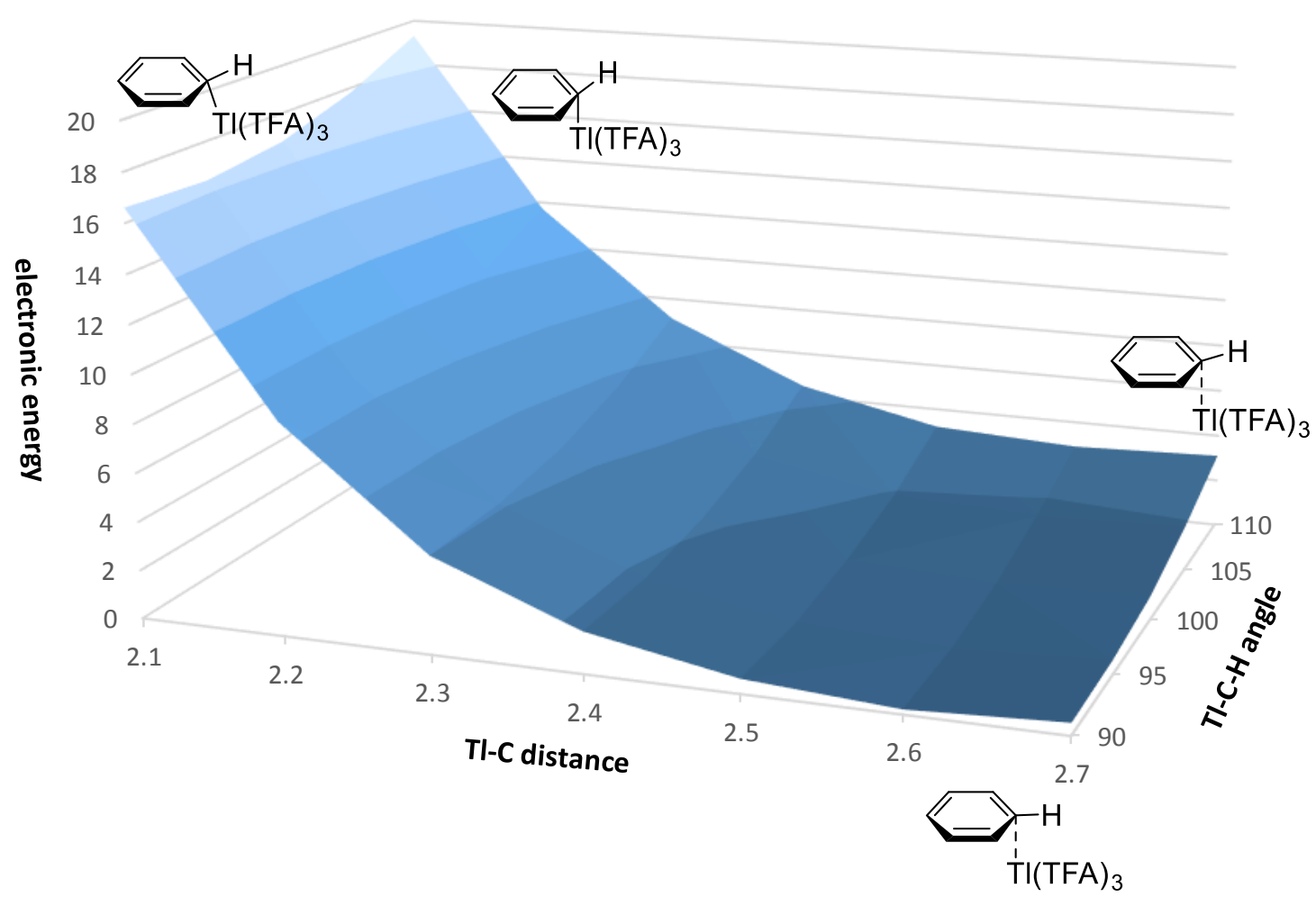

Figure S4. PES generated by scanning the TI-C distance in increments of $0.1 \AA$ and scanning the $\mathrm{TI}-\mathrm{C}-\mathrm{H}(\theta)$ angle in increments of $5^{\circ}$. All other coordinates were unconstrained. $(\mathrm{kcal} / \mathrm{mol})$

The following plots (Chart S1) of different combinations of bond lengths and angles also indicate that a Wheland-type structure is not an energy minimum. 

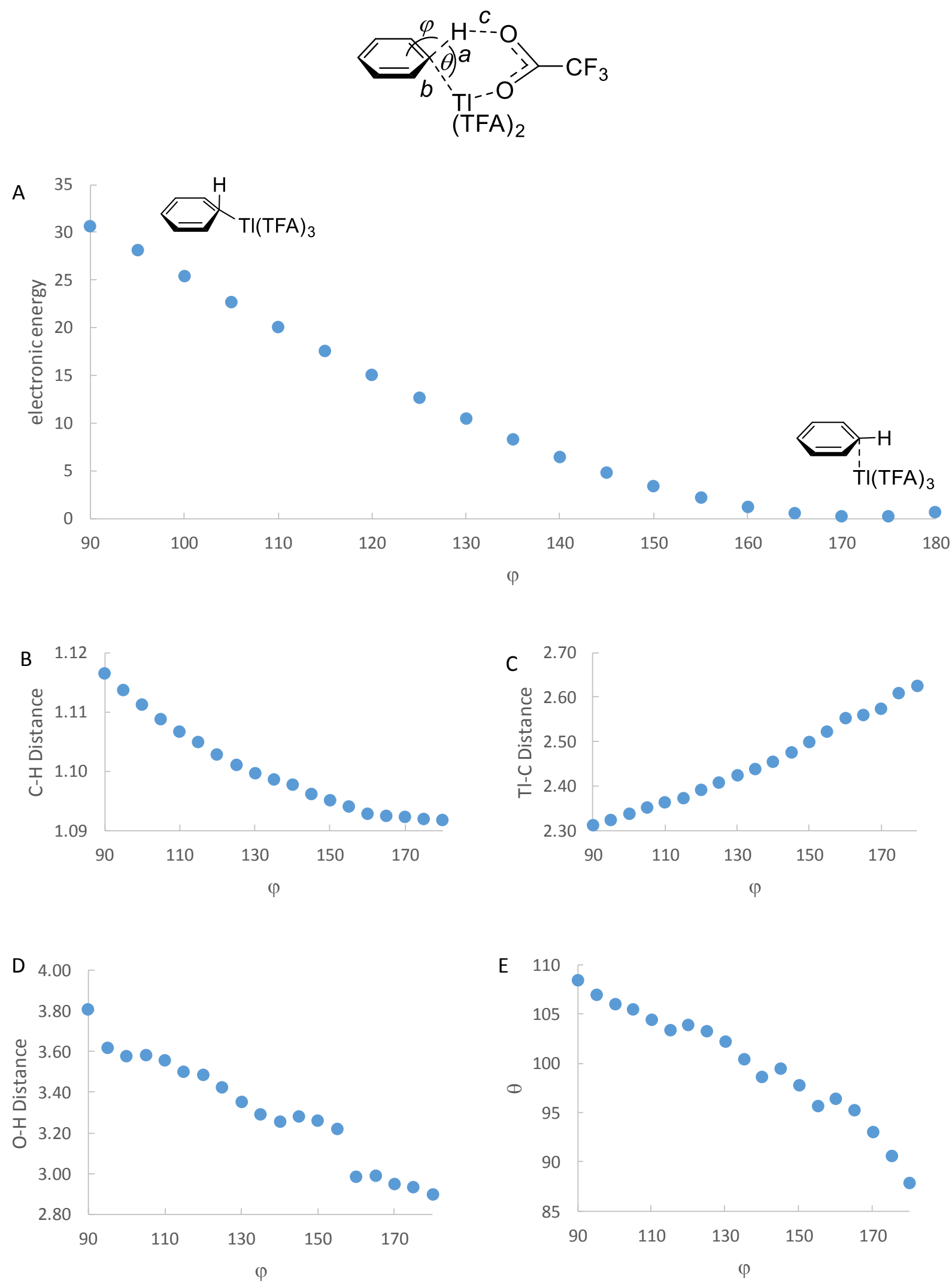
Chart S1. A) Potential energy scan generated by varying the angle between the reactive hydrogen atom and the arene plane $(\varphi)$ in increments of $5^{\circ}$, while all other internal coordinates were allowed to relax. The same angle is $\sim 173^{\circ}$ in the $\pi$ complex structure, and $\sim 128^{\circ}$ in the $\mathrm{C}-\mathrm{H}$ activation transition state structure. B) Plot showing the effect of $\varphi$ on C-H distance (a). C) Plot showing the effect of $\varphi$ on TI-C distance (b). D) Plot showing the effect of $\varphi$ on O-H distance (c). E) Plot showing the effect of $\varphi$ on TI-C-H angle $(\theta)$.

\section{Arene Coordination versus Activation Barriers}

Plot A in Figure S5 shows the free energies for $\pi$-complex formation between methyl-substituted arenes and $\mathrm{TI}(\mathrm{TFA})_{3}$. Plot $\mathrm{B}$ shows the trend in $\Delta G^{\ddagger}$ for TS1 plotted against the $\pi$-complex, which is relatively constant.
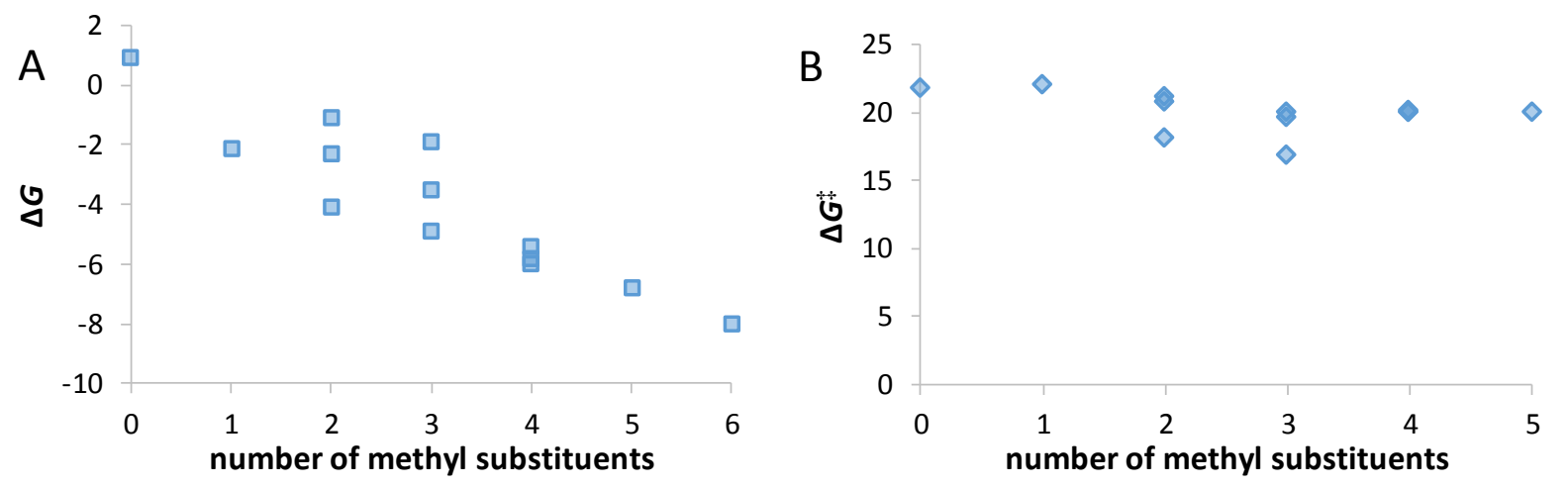

Figure S5. (kcal/mol)

\section{SMD Solvated (U)M06/Def2-TZVPPD//(U)M06/6-31+G(d,p)[LANL2DZdp] XYZ Coordinates and Thermochemistry}

Energies are given in hartrees and coordinates in $\AA$.

$\begin{array}{llll}\mathrm{TI}(\mathrm{TFA})_{3}(1 \mathrm{C}) & & \\ \text { Electronic Energy } & -1751.24440248 \\ \text { Electronic and Zero-point Energy } & -1751.15915218 \\ \text { Enthalpy } & & -1751.13572018 \\ \text { Free Energy } & & -1751.21750218 \\ \mathrm{TI} & 0.040898 & -0.220122 & -0.080699 \\ \mathrm{O} & -1.966183 & -0.925304 & -1.077646 \\ \mathrm{C} & -2.450677 & -1.202423 & 0.05002 \\ \mathrm{O} & 1.614016 & -1.937675 & -0.115713 \\ \mathrm{C} & 2.575885 & -1.124385 & -0.02783 \\ \mathrm{O} & -0.124688 & 1.850107 & 1.059602 \\ \mathrm{C} & -0.082874 & 2.463747 & -0.034664 \\ \mathrm{C} & -0.133641 & 4.000854 & 0.040112 \\ \mathrm{C} & 3.98224 & -1.745809 & 0.040067\end{array}$




$\begin{array}{llll}\text { C } & -3.892176 & -1.742773 & 0.058067 \\ \text { O } & -0.009603 & 1.907175 & -1.153749 \\ \text { O } & -1.834487 & -1.066143 & 1.129387 \\ \text { O } & 2.437585 & 0.111764 & 0.029937 \\ \text { F } & -0.104712 & 4.560836 & -1.162495 \\ \text { F } & 0.904916 & 4.453625 & 0.743687 \\ \text { F } & -1.253128 & 4.382344 & 0.657534 \\ \text { F } & 4.932196 & -0.820792 & 0.031215 \\ F & 4.091136 & -2.456633 & 1.1644 \\ F & 4.176107 & -2.564304 & -0.992756 \\ F & -3.953315 & -2.863183 & -0.662266 \\ F & -4.319066 & -2.00639 & 1.285573 \\ F & -4.710206 & -0.845765 & -0.492586\end{array}$

\begin{tabular}{|c|c|c|c|}
\hline \multicolumn{4}{|c|}{ TI(TFA)з (1D) } \\
\hline \multicolumn{2}{|c|}{ Electronic Energy } & \multicolumn{2}{|c|}{-1751.246314} \\
\hline \multicolumn{2}{|c|}{ Electronic and Zero-point Energy } & \multicolumn{2}{|c|}{-1751.161019} \\
\hline \multicolumn{2}{|c|}{ Enthalpy } & \multicolumn{2}{|c|}{-1751.137515} \\
\hline \multicolumn{2}{|c|}{ Free Energy } & \multicolumn{2}{|c|}{-1751.220433} \\
\hline $\mathrm{TI}$ & -0.105807 & 0.018091 & 0.00000 \\
\hline O & -1.064082 & -1.207941 & 1.741624 \\
\hline O & 1.120226 & -0.865826 & 1.829888 \\
\hline C & 0.059004 & -1.337844 & 2.293602 \\
\hline $\mathrm{O}$ & -1.064082 & -1.207941 & -1.741624 \\
\hline O & 1.120226 & -0.865826 & -1.829888 \\
\hline C & 0.059004 & -1.337844 & -2.293602 \\
\hline $\mathrm{O}$ & -1.146678 & 2.124046 & 0.000000 \\
\hline $\mathrm{O}$ & 1.064853 & 2.068268 & 0.000000 \\
\hline C & -0.024877 & 2.687533 & 0.000000 \\
\hline C & 0.047308 & 4.224368 & 0.000000 \\
\hline C & 0.085174 & -2.131282 & 3.611369 \\
\hline C & 0.085174 & -2.131282 & -3.611369 \\
\hline $\mathrm{F}$ & -1.156771 & 4.780595 & 0.000000 \\
\hline$F$ & 0.707522 & 4.640631 & 1.080751 \\
\hline $\mathrm{F}$ & 0.707522 & 4.640631 & -1.080751 \\
\hline$F$ & -0.720028 & -1.551539 & 4.501936 \\
\hline $\mathrm{F}$ & -0.720028 & -1.551539 & -4.501936 \\
\hline$F$ & -0.355647 & -3.371123 & 3.394399 \\
\hline$F$ & -0.355647 & -3.371123 & -3.394399 \\
\hline$F$ & 1.305534 & -2.200795 & 4.125471 \\
\hline $\mathrm{F}$ & 1.305534 & -2.200795 & -4.125471 \\
\hline
\end{tabular}




\begin{tabular}{|c|c|c|c|}
\hline \multicolumn{4}{|c|}{$\mathrm{TI}(\mathrm{TFA})_{3}(1 \mathrm{E})$} \\
\hline \multicolumn{2}{|c|}{ Electronic Energy } & \multicolumn{2}{|c|}{-1751.245496} \\
\hline \multicolumn{2}{|c|}{ Electronic and Zero-point Energy } & \multicolumn{2}{|c|}{-1751.160224} \\
\hline \multicolumn{2}{|c|}{ Enthalpy } & \multicolumn{2}{|c|}{-1751.136738} \\
\hline \multicolumn{2}{|c|}{ Free Energy } & \multicolumn{2}{|c|}{-1751.219728} \\
\hline $\mathrm{Tl}$ & -0.117834 & 0.016438 & 0.000000 \\
\hline $\mathrm{O}$ & -1.063599 & -1.229865 & 1.756770 \\
\hline $\mathrm{O}$ & 1.118289 & -0.861599 & 1.847515 \\
\hline C & 0.061105 & -1.344530 & 2.307863 \\
\hline $\mathrm{O}$ & -1.063599 & -1.229865 & -1.756770 \\
\hline O & 1.118289 & -0.861599 & -1.847515 \\
\hline C & 0.061105 & -1.344530 & -2.307863 \\
\hline O & -1.150321 & 2.146509 & 0.000000 \\
\hline O & 1.063075 & 2.079301 & 0.000000 \\
\hline C & -0.024741 & 2.701086 & 0.000000 \\
\hline C & 0.054908 & 4.238416 & 0.000000 \\
\hline C & 0.094227 & -2.136641 & 3.627215 \\
\hline C & 0.094227 & -2.136641 & -3.627215 \\
\hline $\mathrm{F}$ & -1.146435 & 4.800870 & 0.000000 \\
\hline $\mathrm{F}$ & 0.716981 & 4.652052 & 1.080736 \\
\hline $\mathrm{F}$ & 0.716981 & 4.652052 & -1.080736 \\
\hline $\mathrm{F}$ & -0.713581 & -1.560466 & 4.518117 \\
\hline $\mathrm{F}$ & -0.713581 & -1.560466 & -4.518117 \\
\hline $\mathrm{F}$ & -0.338922 & -3.379630 & 3.413292 \\
\hline $\mathrm{F}$ & -0.338922 & -3.379630 & -3.413292 \\
\hline $\mathrm{F}$ & 1.315549 & -2.197808 & 4.140458 \\
\hline $\mathrm{F}$ & 1.315549 & -2.197808 & -4.140458 \\
\hline \multicolumn{4}{|c|}{ 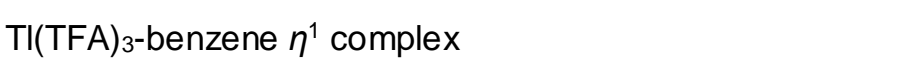 } \\
\hline \multicolumn{2}{|c|}{ Electronic Energy } & \multicolumn{2}{|c|}{-1983.417513} \\
\hline \multicolumn{2}{|c|}{ Electronic and Zero-point Energy } & \multicolumn{2}{|c|}{-1983.230865} \\
\hline \multicolumn{2}{|c|}{ Enthalpy } & \multicolumn{2}{|c|}{-1983.201332} \\
\hline \multicolumn{2}{|c|}{ Free Energy } & \multicolumn{2}{|c|}{-1983.296853} \\
\hline $\mathrm{Tl}$ & 0.386117 & 0.477585 & 3.666029 \\
\hline $\mathrm{O}$ & 2.640681 & 0.963669 & 4.452121 \\
\hline C & 2.510158 & 2.142564 & 4.053227 \\
\hline $\mathrm{O}$ & -1.599226 & 1.715339 & 3.849988 \\
\hline C & -2.351318 & 0.725607 & 3.628148 \\
\hline O & 0.017376 & -0.394207 & 5.87817 \\
\hline C & 0.438746 & -1.517266 & 5.519326 \\
\hline C & 3.723336 & 3.073886 & 4.22521 \\
\hline \multirow[t]{2}{*}{ C } & -3.860909 & 1.030911 & 3.624227 \\
\hline & & & S10 \\
\hline
\end{tabular}




\begin{tabular}{|c|c|c|c|}
\hline C & 0.490374 & -2.65748 & 6.552715 \\
\hline $\mathrm{O}$ & 1.485418 & 2.594516 & 3.489823 \\
\hline O & -1.957633 & -0.429507 & 3.393589 \\
\hline O & 0.849672 & -1.790321 & 4.366081 \\
\hline $\mathrm{F}$ & 3.416365 & 4.347929 & 4.009399 \\
\hline $\mathrm{F}$ & 4.236927 & 2.965886 & 5.448731 \\
\hline$F$ & 4.666053 & 2.715637 & 3.344126 \\
\hline $\mathrm{F}$ & -4.144481 & 1.84804 & 2.60297 \\
\hline$F$ & -4.221012 & 1.641955 & 4.753542 \\
\hline $\mathrm{F}$ & -4.594417 & -0.068525 & 3.490716 \\
\hline $\mathrm{F}$ & -0.095982 & -2.327851 & 7.697888 \\
\hline $\mathrm{F}$ & -0.111162 & -3.747552 & 6.07235 \\
\hline $\mathrm{F}$ & 1.765053 & -2.968335 & 6.808881 \\
\hline C & 0.74503 & 1.879876 & 0.341656 \\
\hline C & 2.786604 & 0.68521 & 0.897687 \\
\hline C & -0.013519 & 0.790136 & 0.751436 \\
\hline $\mathrm{H}$ & 0.25375 & 2.773412 & -0.034711 \\
\hline C & 2.042457 & -0.408701 & 1.32341 \\
\hline $\mathrm{H}$ & 3.872486 & 0.653528 & 0.944891 \\
\hline C & 0.631888 & -0.363813 & 1.255832 \\
\hline $\mathrm{H}$ & -1.099878 & 0.810879 & 0.683583 \\
\hline $\mathrm{H}$ & 2.531621 & -1.300344 & 1.711733 \\
\hline $\mathrm{H}$ & 0.04897 & -1.261272 & 1.474977 \\
\hline C & 2.138192 & 1.821094 & 0.408473 \\
\hline $\mathrm{H}$ & 2.727369 & 2.676857 & 0.085181 \\
\hline
\end{tabular}

\begin{tabular}{|c|c|c|c|}
\hline \multicolumn{4}{|c|}{ TI(TFA) ${ }_{3}$-benzene $\eta^{2}$ complex } \\
\hline \multicolumn{2}{|c|}{ Electronic Energy } & \multicolumn{2}{|c|}{-1983.416624} \\
\hline \multicolumn{2}{|c|}{ Electronic and Zero-point Energy } & \multicolumn{2}{|c|}{-1983.228929} \\
\hline \multicolumn{2}{|c|}{ Enthalpy } & \multicolumn{2}{|c|}{-1983.199918} \\
\hline \multicolumn{2}{|c|}{ Free Energy } & \multicolumn{2}{|c|}{-1983.292242} \\
\hline TI & 0.221699 & 0.601831 & 3.596499 \\
\hline $\mathrm{O}$ & 2.569609 & 0.913965 & 3.998003 \\
\hline C & 2.406756 & 2.155433 & 4.058776 \\
\hline $\mathrm{O}$ & -1.791221 & 1.836738 & 3.75773 \\
\hline C & -2.501508 & 0.81025 & 3.589665 \\
\hline $\mathrm{O}$ & 0.067268 & -0.236949 & 5.836027 \\
\hline C & 0.436293 & -1.372441 & 5.45402 \\
\hline C & 3.676143 & 2.994893 & 4.28982 \\
\hline C & -4.024116 & 1.042752 & 3.603682 \\
\hline C & 0.544267 & -2.508966 & 6.486312 \\
\hline $\mathrm{O}$ & 1.304601 & 2.73382 & 3.929072 \\
\hline
\end{tabular}




\begin{tabular}{|c|c|c|c|}
\hline $\mathrm{O}$ & -2.054679 & -0.337806 & 3.398974 \\
\hline 0 & 0.72968 & -1.667876 & 4.272679 \\
\hline$F$ & 3.421063 & 4.297083 & 4.334786 \\
\hline $\mathrm{F}$ & 4.260596 & 2.639362 & 5.434897 \\
\hline$F$ & 4.540956 & 2.770658 & 3.29515 \\
\hline $\mathrm{F}$ & -4.35562 & 1.868733 & 2.605714 \\
\hline$F$ & -4.395634 & 1.611153 & 4.751626 \\
\hline $\mathrm{F}$ & -4.708404 & -0.085433 & 3.454603 \\
\hline $\mathrm{F}$ & 0.158822 & -2.129892 & 7.699442 \\
\hline $\mathrm{F}$ & -0.214741 & -3.540392 & 6.10782 \\
\hline $\mathrm{F}$ & 1.809114 & -2.933128 & 6.560026 \\
\hline C & 0.869336 & 1.873422 & 0.698582 \\
\hline C & 2.906357 & 0.572329 & 0.773896 \\
\hline C & 0.125753 & 0.706433 & 0.951638 \\
\hline $\mathrm{H}$ & 0.354829 & 2.823475 & 0.574951 \\
\hline C & 2.185454 & -0.590107 & 1.029102 \\
\hline $\mathrm{H}$ & 3.991563 & 0.529957 & 0.709591 \\
\hline C & 0.79278 & -0.533262 & 1.111955 \\
\hline $\mathrm{H}$ & -0.964809 & 0.731991 & 0.926291 \\
\hline $\mathrm{H}$ & 2.69826 & -1.538792 & 1.166085 \\
\hline $\mathrm{H}$ & 0.209677 & -1.438608 & 1.281973 \\
\hline C & 2.25306 & 1.799249 & 0.613559 \\
\hline $\mathrm{H}$ & 2.831897 & 2.699467 & 0.418459 \\
\hline \multicolumn{4}{|c|}{$\left[\mathrm{TI}(\mathrm{TFA})_{2}(\mathrm{TFAH})\right]^{+}$} \\
\hline \multicolumn{2}{|c|}{ Electronic Energy } & \multicolumn{2}{|c|}{-1751.64137101} \\
\hline \multicolumn{2}{|c|}{ Electronic and Zero-point Energy } & \multicolumn{2}{|c|}{-1751.54238182} \\
\hline \multicolumn{2}{|c|}{ Enthalpy } & \multicolumn{2}{|c|}{-1751.51932882} \\
\hline \multicolumn{2}{|c|}{ Free Energy } & \multicolumn{2}{|c|}{-1751.59819782} \\
\hline $\mathrm{TI}$ & -0.117449 & -0.903609 & 0.04829 \\
\hline $\mathrm{O}$ & 0.553802 & 1.364334 & -0.183401 \\
\hline C & 0.007873 & 2.440361 & 0.022357 \\
\hline $\mathrm{O}$ & 1.902746 & -1.337985 & -1.032598 \\
\hline C & 2.48414 & -1.354887 & 0.079807 \\
\hline $\mathrm{O}$ & -2.189855 & 0.069513 & 0.653281 \\
\hline C & -2.785098 & -0.857188 & 0.047434 \\
\hline C & -4.325426 & -0.814068 & 0.010581 \\
\hline C & 4.01058 & -1.572862 & 0.068748 \\
\hline C & 0.812864 & 3.750896 & -0.116398 \\
\hline $\mathrm{O}$ & -2.178016 & -1.797615 & -0.516846 \\
\hline $\mathrm{O}$ & -1.214938 & 2.680386 & 0.359419 \\
\hline \multirow[t]{2}{*}{ O } & 1.885453 & -1.204052 & 1.173357 \\
\hline & & & S12 \\
\hline
\end{tabular}




$\begin{array}{llll}\text { F } & -4.825423 & -1.843337 & -0.650971 \\ \text { F } & -4.712153 & 0.31318 & -0.580622 \\ \text { F } & -4.791712 & -0.82549 & 1.254869 \\ \text { F } & 4.523423 & -1.496054 & 1.286127 \\ \text { F } & 4.274088 & -2.774558 & -0.437846 \\ \text { F } & 4.580997 & -0.650972 & -0.700206 \\ \text { F } & 2.072541 & 3.495631 & -0.42363 \\ \text { F } & 0.275 & 4.504782 & -1.071901 \\ \text { F } & 0.769889 & 4.423477 & 1.030318 \\ \text { H } & -1.766248 & 1.867341 & 0.467604\end{array}$

$\begin{array}{llll}\text { [(TFAH)(TFA)] } & & \\ \text { Electronic Energy } & & -1053.21917964 \\ \text { Electronic and Zero-point Energy } & -1053.15470640 \\ \text { Enthalpy } & & -1053.14056940 \\ \text { Free Energy } & & -1053.19822640 \\ \text { C } & -0.453954 & 0.127479 & -0.082566 \\ \mathrm{C} & 0.954347 & 0.763117 & -0.018573 \\ \text { O } & 1.666633 & 0.375017 & 0.966195 \\ \text { H } & 2.758362 & 0.896678 & 0.962322 \\ \text { O } & 1.230193 & 1.559432 & -0.907272 \\ \text { F } & -0.721962 & -0.706433 & 0.923675 \\ \text { F } & -0.597963 & -0.557337 & -1.226501 \\ \text { F } & -1.391969 & 1.086434 & -0.072273 \\ \text { O } & 3.818458 & 1.446744 & 0.952911 \\ \text { C } & 4.532629 & 1.124991 & -0.058863 \\ \text { C } & 5.815389 & 1.984657 & -0.134153 \\ \text { O } & 4.32152 & 0.280911 & -0.916365 \\ \text { F } & 5.505177 & 3.278887 & -0.301192 \\ \text { F } & 6.527368 & 1.888072 & 0.998711 \\ \text { F } & 6.608658 & 1.624809 & -1.142467\end{array}$

$\begin{array}{llll}\text { TI(TFA) }{ }_{4}^{-} & & \\ \text {Electronic Energy } & & -2277.669558 & \\ \text { Electronic and Zero-point Energy } & -2277.556146 & \\ \text { Enthalpy } & & -2277.525191 & \\ \text { Free Energy } & -2277.62417 & \\ \text { TI } & 0.047786 & -0.035381 & 0.037441 \\ \text { O } & -1.072532 & 1.53284 & 1.451936 \\ \text { C } & -0.46991 & 2.510917 & 0.940058 \\ \text { O } & 0.203719 & -1.359474 & 2.030846 \\ \text { C } & 0.465344 & -2.392561 & 1.366414\end{array}$




$\begin{array}{llll}\text { O } & -2.202585 & -1.012066 & -0.142499 \\ \text { C } & -2.423471 & -0.276712 & -1.128492 \\ \text { O } & 1.590661 & 0.009254 & -1.796163 \\ \text { C } & 2.568851 & 0.086416 & -1.012223 \\ \text { O } & 2.488398 & 0.104972 & 0.233391 \\ \text { O } & 0.392205 & 2.428078 & 0.044322 \\ \text { O } & 0.573982 & -2.437281 & 0.12362 \\ \text { O } & -1.599882 & 0.53135 & -1.619324 \\ \text { C } & 3.954207 & 0.180528 & -1.67681 \\ \text { C } & -3.805179 & -0.410487 & -1.793507 \\ \text { C } & -0.877152 & 3.897897 & 1.470102 \\ \text { C } & 0.664878 & -3.681485 & 2.184326 \\ F & 4.145351 & -0.850145 & -2.505238 \\ \text { F } & 4.034165 & 1.305208 & -2.395643 \\ F & -4.046182 & 0.561239 & -2.669175 \\ F & -3.874196 & -1.580133 & -2.441647 \\ F & -4.775324 & -0.39356 & -0.877297 \\ F & -2.138731 & 4.162018 & 1.110182 \\ F & -0.81788 & 3.928458 & 2.803999 \\ F & -0.100773 & 4.869204 & 0.999123 \\ F & -0.413473 & -3.924427 & 2.934561 \\ F & 0.881381 & -4.744671 & 1.415423 \\ F & 1.712538 & -3.542279 & 3.004213 \\ F & 4.944281 & 0.185585 & -0.788949\end{array}$

\begin{tabular}{|c|c|c|c|}
\hline \multicolumn{2}{|c|}{ Electronic Energy } & \multicolumn{2}{|c|}{-231.4632001} \\
\hline \multicolumn{2}{|c|}{ Electronic and Zero-point Energy } & \multicolumn{2}{|c|}{-231.3762078} \\
\hline \multicolumn{2}{|c|}{ Enthalpy } & \multicolumn{2}{|c|}{-231.3708358} \\
\hline \multicolumn{2}{|c|}{ Free Energy } & \multicolumn{2}{|c|}{-231.4036228} \\
\hline C & 0.00 & 0.00 & 1.395475 \\
\hline C & 0.00 & 1.224938 & 0.770497 \\
\hline C & 0.00 & 1.212724 & -0.630744 \\
\hline C & 0.00 & 0.00 & -1.321005 \\
\hline C & 0.00 & -1.212724 & -0.630744 \\
\hline C & 0.00 & -1.224938 & 0.770497 \\
\hline $\mathrm{H}$ & 0.00 & 2.164238 & 1.321059 \\
\hline $\mathrm{H}$ & 0.00 & 2.154124 & -1.178287 \\
\hline $\mathrm{H}$ & 0.00 & 0.00 & -2.409399 \\
\hline $\mathrm{H}$ & 0.00 & -2.154124 & -1.178287 \\
\hline $\mathrm{H}$ & 0.00 & -2.164238 & 1.321059 \\
\hline
\end{tabular}




$\begin{array}{llll}\text { TI(TFA) }{ }_{2} \text { TFAH }(\mathrm{TI}(\mathrm{II})) & & \\ \text { Electronic Energy } & & -1751.64137101 \\ \text { Electronic and Zero-point Energy } & -1751.54238182 \\ \text { Enthalpy } & & -1751.51932882 \\ \text { Free Energy } & & -1751.59819782 \\ \text { TI } & -0.345635 & -1.937213 & -0.282854 \\ \mathrm{O} & -0.261297 & 0.605804 & -1.173533 \\ \mathrm{C} & -0.031545 & 1.536294 & -0.426672 \\ \mathrm{O} & 2.109896 & -1.746753 & -0.786341 \\ \mathrm{C} & 2.325057 & -0.961364 & 0.171647 \\ \mathrm{O} & -2.07592 & -0.436469 & 1.036957 \\ \mathrm{C} & -2.935555 & -0.724208 & 0.170523 \\ \mathrm{C} & -4.226082 & 0.127462 & 0.204101 \\ \mathrm{C} & 3.801058 & -0.508853 & 0.29287 \\ \mathrm{C} & 1.034587 & 2.595582 & -0.769609 \\ \mathrm{O} & -2.826195 & -1.566626 & -0.741403 \\ \mathrm{O} & -0.571819 & 1.795422 & 0.72822 \\ \mathrm{O} & 1.496842 & -0.521062 & 0.985746 \\ \mathrm{~F} & -5.186061 & -0.363366 & -0.571601 \\ \mathrm{~F} & -3.92416 & 1.359565 & -0.232157 \\ \mathrm{~F} & -4.703122 & 0.236596 & 1.444008 \\ \mathrm{~F} & 4.015402 & 0.257825 & 1.356635 \\ \mathrm{~F} & 4.603194 & -1.57467 & 0.382249 \\ \mathrm{~F} & 4.150971 & 0.180656 & -0.798151 \\ \mathrm{~F} & 1.593989 & 2.339835 & -1.944211 \\ \mathrm{~F} & 0.494627 & 3.814743 & -0.808402 \\ \mathrm{~F} & 1.987541 & 2.588963 & 0.164342 \\ \mathrm{H} & -1.214494 & 1.081708 & 0.988064\end{array}$

$\mathrm{TI}(\mathrm{TFA})_{3}{ }^{-}$radical anion

\begin{tabular}{llll} 
Electronic Energy & \multicolumn{3}{c}{-1751.430096} \\
Electronic and Zero-point Energy & -1751.347319 & \\
Enthalpy & & -1751.322699 & \\
Free Energy & & -1751.411305 \\
TI & -0.159132 & -0.30392 & -0.065223 \\
O & -2.573237 & -0.533206 & -1.09552 \\
C & -3.051647 & -0.743654 & 0.039983 \\
O & 1.472399 & -2.341848 & 0.044001 \\
C & 2.483853 & -1.603637 & -0.025527 \\
O & 0.418562 & 1.958624 & 1.072045 \\
C & 0.606831 & 2.506333 & -0.037538 \\
C & 1.112058 & 3.967032 & 0.047676
\end{tabular}




$\begin{array}{llll}\text { C } & 3.827066 & -2.370775 & 0.042712 \\ \text { C } & -4.582974 & -0.97712 & 0.059139 \\ \text { O } & 0.433789 & 2.017554 & -1.17076 \\ \text { O } & -2.450412 & -0.783203 & 1.132229 \\ \text { O } & 2.523058 & -0.365415 & -0.12583 \\ F & 1.355696 & 4.505985 & -1.146636 \\ F & 2.241668 & 4.033834 & 0.763362 \\ F & 0.200322 & 4.735561 & 0.662206 \\ F & 4.890111 & -1.58246 & -0.111965 \\ F & 3.944341 & -2.985041 & 1.229036 \\ F & 3.879551 & -3.314549 & -0.906642 \\ F & -4.914457 & -1.978693 & -0.765934 \\ F & -5.049149 & -1.277969 & 1.270407 \\ F & -5.223048 & 0.122042 & -0.363274\end{array}$

\begin{tabular}{llll} 
Benzene & & \\
Electronic Energy & & \multicolumn{2}{l}{-232.148956617} \\
Electronic and Zero-point Energy & \multicolumn{2}{l}{-232.048961683} \\
Enthalpy & & \multicolumn{2}{l}{-232.043573683} \\
Free Energy & & -232.076448683 \\
$\mathrm{C}$ & 0.051356 & 1.206844 & 0.000072 \\
$\mathrm{C}$ & 1.445666 & 1.206817 & 0.000584 \\
$\mathrm{C}$ & 2.14291 & 2.414017 & -0.000087 \\
$\mathrm{C}$ & 1.4457 & 3.621476 & -0.001024 \\
$\mathrm{C}$ & 0.051587 & 3.621502 & -0.001423 \\
$\mathrm{C}$ & -0.645757 & 2.41414 & -0.000988 \\
$\mathrm{H}$ & -0.492718 & 0.264278 & 0.000436 \\
$\mathrm{H}$ & 1.989613 & 0.264168 & 0.00136 \\
$\mathrm{H}$ & 3.231327 & 2.414119 & 0.000176 \\
$\mathrm{H}$ & 1.990017 & 4.563897 & -0.001446 \\
$\mathrm{H}$ & -0.492587 & 4.564014 & -0.002257 \\
$\mathrm{H}$ & -1.734181 & 2.41431 & -0.001315
\end{tabular}

Toluene

\begin{tabular}{llll} 
Electronic Energy & & \multicolumn{2}{l}{-271.449337309} \\
Electronic and Zero-point Energy & -271.322230554 \\
Enthalpy & & -271.315134554 \\
Free Energy & & -271.352133554 \\
C & 0.046111 & 1.198216 & -0.010296 \\
C & 1.440404 & 1.205876 & -0.012019 \\
C & 2.134262 & 2.413821 & 0.00198 \\
C & 1.45236 & 3.635872 & 0.015841
\end{tabular}




$\begin{array}{llll}\mathrm{C} & 0.053798 & 3.613954 & 0.02135 \\ \mathrm{C} & -0.6452 & 2.408137 & 0.007365 \\ \mathrm{H} & -0.497465 & 0.255284 & -0.018721 \\ \mathrm{H} & 1.990599 & 0.266303 & -0.021086 \\ \mathrm{H} & 3.22487 & 2.4123 & 0.003556 \\ \mathrm{H} & -0.4932 & 4.557013 & 0.037734 \\ \mathrm{H} & -1.734105 & 2.414432 & 0.01334 \\ \mathrm{C} & 2.20338 & 4.933327 & -0.003209 \\ \mathrm{H} & 2.466326 & 5.223234 & -1.029769 \\ \mathrm{H} & 1.608217 & 5.74965 & 0.422088 \\ \mathrm{H} & 3.141403 & 4.864929 & 0.560482\end{array}$

\begin{tabular}{llll} 
O-Xylene & \multicolumn{3}{c}{-310.7504733} \\
Electronic Energy & \multicolumn{3}{c}{-310.5956188} \\
Electronic and Zero-point Energy & -310.5871288 \\
Enthalpy & & -310.6270968 \\
Free Energy & & \\
C & 0.478146 & 0.704672 & -0.000012 \\
$\mathrm{C}$ & 0.478151 & -0.704656 & -0.000036 \\
$\mathrm{C}$ & -0.743546 & -1.382618 & -0.000087 \\
$\mathrm{C}$ & -1.956611 & -0.695719 & -0.000018 \\
$\mathrm{C}$ & -1.956612 & 0.695707 & 0.00007 \\
$\mathrm{C}$ & -0.743554 & 1.382619 & 0.000037 \\
$\mathrm{H}$ & -0.741495 & -2.473176 & -0.000202 \\
$\mathrm{H}$ & -2.895174 & -1.247307 & -0.000077 \\
$\mathrm{H}$ & -2.895175 & 1.247295 & 0.000121 \\
$\mathrm{H}$ & -0.741515 & 2.473177 & 0.00007 \\
$\mathrm{C}$ & 1.76892 & 1.4663 & -0.000063 \\
$\mathrm{H}$ & 2.382501 & 1.229118 & 0.880183 \\
$\mathrm{H}$ & 2.382024 & 1.229768 & -0.880815 \\
$\mathrm{H}$ & 1.590695 & 2.547343 & 0.00037 \\
$\mathrm{C}$ & 1.768924 & -1.466299 & 0.000079 \\
$\mathrm{H}$ & 2.382639 & -1.22914 & -0.880064 \\
$\mathrm{H}$ & 2.381909 & -1.229776 & 0.880927 \\
$\mathrm{H}$ & 1.590678 & -2.54734 & -0.000337 \\
& & &
\end{tabular}

$m$-Xylene

\begin{tabular}{|c|c|}
\hline Electronic Energy & -310.7497434 \\
\hline Electronic and Zero-point Energy & -310.5953076 \\
\hline Enthalpy & -310.5865226 \\
\hline Free Energy & -310.6275006 \\
\hline-1.2181 & -0.278941 \\
\hline
\end{tabular}




$\begin{array}{llll}\mathrm{C} & 0.005555 & -0.949597 & -0.00209 \\ \mathrm{C} & 1.226965 & -0.263567 & 0.004579 \\ \mathrm{C} & 1.205889 & 1.132281 & 0.006388 \\ \mathrm{C} & -0.008335 & 1.820254 & 0.000193 \\ \mathrm{C} & -1.210856 & 1.122341 & -0.006646 \\ \mathrm{H} & 2.145726 & 1.684464 & 0.011384 \\ \mathrm{H} & -0.012863 & 2.90939 & 0.000506 \\ \mathrm{H} & -2.157748 & 1.663312 & -0.010819 \\ \mathrm{C} & -2.515873 & -1.030312 & 0.004454 \\ \mathrm{H} & -2.364559 & -2.10136 & -0.171054 \\ \mathrm{H} & -3.03037 & -0.921614 & 0.968834 \\ \mathrm{H} & -3.20314 & -0.655909 & -0.764678 \\ \mathrm{H} & 0.012981 & -2.042069 & -0.003868 \\ \mathrm{C} & 2.519064 & -1.024985 & -0.002013 \\ \mathrm{H} & 2.543022 & -1.780225 & 0.793865 \\ \mathrm{H} & 2.661887 & -1.560082 & -0.950367 \\ \mathrm{H} & 3.379212 & -0.360754 & 0.137691\end{array}$

$\begin{array}{llll}\text { p-Xylene } & & \\ \text { Electronic Energy } & & -310.7494231 & \\ \text { Electronic and Zero-point Energy } & -310.5953643 & \\ \text { Enthalpy } & & -310.5864023 \\ \text { Free Energy } & & -310.6282633 \\ \text { C } & 1.419422 & 0.006445 & 0.003365 \\ \mathrm{C} & 0.694924 & -1.192883 & 0.001863 \\ \mathrm{C} & -0.694914 & -1.19289 & -0.001927 \\ \mathrm{C} & -1.41942 & 0.00643 & -0.003428 \\ \mathrm{C} & -0.698002 & 1.200923 & -0.001771 \\ \mathrm{C} & 0.697999 & 1.200931 & 0.001708 \\ \mathrm{H} & -1.234815 & 2.150038 & -0.002592 \\ \mathrm{H} & 1.234808 & 2.150048 & 0.002544 \\ \mathrm{C} & 2.918762 & -0.007657 & -0.001032 \\ \mathrm{H} & 3.317558 & -0.604005 & 0.829866 \\ \mathrm{H} & 3.312793 & -0.449763 & -0.925976 \\ \mathrm{H} & 3.331004 & 1.004116 & 0.085112 \\ \mathrm{H} & 1.234133 & -2.141322 & 0.002821 \\ \mathrm{C} & -2.918767 & -0.007658 & 0.001141 \\ \mathrm{H} & -3.317694 & -0.604993 & -0.828985 \\ \mathrm{H} & -3.31267 & -0.448649 & 0.926674 \\ \mathrm{H} & -3.331017 & 1.004019 & -0.08611 \\ \mathrm{H} & -1.234113 & -2.141337 & -0.002865\end{array}$




$\begin{array}{llll}\text { Mesitylene } & & \\ \text { Electronic Energy } & & -350.0499606 & \\ \text { Electronic and Zero-point Energy } & -349.8684311 & \\ \text { Enthalpy } & & -349.8578831 & \\ \text { Free Energy } & & -349.9029791 & \\ \text { C } & -0.722962 & -1.207649 & -0.019737 \\ \mathrm{C} & -1.384478 & 0.021582 & -0.000982 \\ \mathrm{C} & -0.678184 & 1.22903 & 0.019426 \\ \mathrm{C} & 0.717232 & 1.187311 & 0.020572 \\ \mathrm{C} & 1.409905 & -0.028094 & 0.000982 \\ \mathrm{C} & 0.676047 & -1.215774 & -0.01948 \\ \mathrm{H} & 1.20432 & -2.170873 & -0.035994 \\ \mathrm{C} & -1.502248 & -2.489499 & -0.028627 \\ \mathrm{H} & -2.271906 & -2.485898 & -0.810849 \\ \mathrm{H} & -0.852547 & -3.355396 & -0.199139 \\ \mathrm{H} & -2.021641 & -2.646996 & 0.926181 \\ \mathrm{H} & -2.477096 & 0.041129 & -0.003773 \\ \mathrm{H} & 1.281029 & 2.122621 & 0.036064 \\ \mathrm{C} & -1.416224 & 2.535147 & 0.025481 \\ \mathrm{H} & -1.941389 & 2.7002 & -0.924857 \\ \mathrm{H} & -2.177303 & 2.560859 & 0.815712 \\ \mathrm{H} & -0.738475 & 3.381775 & 0.182217 \\ \mathrm{C} & 2.90938 & -0.043083 & 0.012941 \\ \mathrm{H} & 3.30388 & 0.321104 & 0.971179 \\ \mathrm{H} & 3.304616 & -1.053097 & -0.144129 \\ \mathrm{H} & 3.324365 & 0.607102 & -0.767837\end{array}$

1,2,3-trimethylbenzene

\begin{tabular}{llll} 
Electronic Energy & \multicolumn{3}{l}{-350.0488136} \\
Electronic and Zero-point Energy & -349.8665966 & \\
Enthalpy & & -349.8564506 & \\
Free Energy & & -349.9001926 & \\
C & 1.213294 & 0.073092 & -0.003401 \\
C & 0.010627 & -0.6615 & -0.011028 \\
C & -1.221612 & 0.019822 & -0.00423 \\
C & -1.231525 & 1.418896 & -0.001622 \\
C & -0.04573 & 2.145108 & -0.00204 \\
C & 1.17038 & 1.469802 & -0.000153 \\
H & 2.10605 & 2.029394 & 0.005916 \\
C & 2.538546 & -0.629991 & 0.007688 \\
H & 2.630956 & -1.317641 & 0.859435 \\
H & 2.691765 & -1.23283 & -0.898264
\end{tabular}




$\begin{array}{llll}\mathrm{H} & 3.363084 & 0.08868 & 0.069293 \\ \mathrm{H} & -0.06901 & 3.23375 & 0.000061 \\ \mathrm{C} & 0.067763 & -2.161781 & -0.00595 \\ \mathrm{H} & 0.797696 & -2.541862 & -0.731823 \\ \mathrm{H} & 0.374868 & -2.5494 & 0.976684 \\ \mathrm{H} & -0.896068 & -2.619431 & -0.245901 \\ \mathrm{H} & -2.188366 & 1.941348 & 0.003202 \\ \mathrm{C} & -2.528916 & -0.72042 & 0.008453 \\ \mathrm{H} & -2.661399 & -1.341745 & -0.887427 \\ \mathrm{H} & -2.616633 & -1.389138 & 0.875093 \\ \mathrm{H} & -3.369907 & -0.019302 & 0.047429\end{array}$

\begin{tabular}{llll} 
1,2,4-Trimethylbenzene & \\
Electronic Energy & \multicolumn{3}{l}{-350.0505305} \\
Electronic and Zero-point Energy & -349.8684978 \\
Enthalpy & & -349.8582818 \\
Free Energy & & -349.9021728 \\
C & -1.128509 & -0.588097 & 0.000011 \\
$\mathrm{C}$ & -0.698325 & 0.754794 & -0.003759 \\
$\mathrm{C}$ & 0.669125 & 1.030442 & -0.005807 \\
$\mathrm{C}$ & 1.638057 & 0.020206 & -0.003438 \\
$\mathrm{C}$ & 1.199549 & -1.303254 & -0.002769 \\
$\mathrm{C}$ & -0.164399 & -1.596183 & -0.000766 \\
$\mathrm{H}$ & -0.488119 & -2.637663 & -0.000187 \\
$\mathrm{C}$ & -2.588998 & -0.92564 & 0.00312 \\
$\mathrm{H}$ & -3.105355 & -0.501691 & 0.875627 \\
$\mathrm{H}$ & -3.101777 & -0.530879 & -0.885088 \\
$\mathrm{H}$ & -2.744747 & -2.010262 & 0.020078 \\
$\mathrm{H}$ & 0.995809 & 2.073048 & -0.008678 \\
$\mathrm{H}$ & 1.928589 & -2.114038 & -0.003907 \\
$\mathrm{C}$ & -1.696143 & 1.873017 & 0.00127 \\
$\mathrm{H}$ & -2.400413 & 1.79802 & -0.838432 \\
$\mathrm{H}$ & -2.303332 & 1.870334 & 0.917408 \\
$\mathrm{H}$ & -1.200679 & 2.847919 & -0.064841 \\
$\mathrm{C}$ & 3.096512 & 0.36877 & 0.005422 \\
$\mathrm{H}$ & 3.355207 & 1.038759 & -0.824825 \\
$\mathrm{H}$ & 3.379535 & 0.887834 & 0.931059 \\
$\mathrm{H}$ & 3.724063 & -0.525705 & -0.077918
\end{tabular}

1,2,4,5-Tetramethylbenzene (durene)

Electronic Energy $\quad-389.3512095$

Electronic and Zero-point Energy $\quad-389.141491$ 


\begin{tabular}{|c|c|c|c|}
\hline \multirow{2}{*}{$\begin{array}{l}\text { Enthalpy } \\
\text { Free Energy }\end{array}$} & \multicolumn{3}{|c|}{-389.129795} \\
\hline & & -389.1768 & \\
\hline $\mathrm{C}$ & -1.226299 & -0.702614 & -0.000043 \\
\hline C & -1.226296 & 0.702604 & -0.000027 \\
\hline C & 0.000014 & 1.37137 & -0.000073 \\
\hline C & 1.226314 & 0.7026 & -0.000029 \\
\hline C & 1.226301 & -0.702629 & 0.000085 \\
\hline C & 0.000005 & -1.371386 & 0.000085 \\
\hline $\mathrm{H}$ & -0.00002 & -2.463765 & 0.000203 \\
\hline C & -2.513679 & -1.470797 & -0.000092 \\
\hline $\mathrm{H}$ & -3.12945 & -1.237489 & 0.879919 \\
\hline $\mathrm{H}$ & -3.128645 & -1.238621 & -0.880989 \\
\hline $\mathrm{H}$ & -2.330609 & -2.551257 & 0.000645 \\
\hline C & -2.513651 & 1.47082 & 0.000093 \\
\hline $\mathrm{H}$ & -3.129401 & 1.237783 & -0.880003 \\
\hline $\mathrm{H}$ & -3.12864 & 1.23842 & 0.880913 \\
\hline $\mathrm{H}$ & -2.330532 & 2.55127 & -0.00031 \\
\hline $\mathrm{H}$ & -0.000012 & 2.463745 & -0.000106 \\
\hline C & 2.513665 & 1.470832 & -0.000037 \\
\hline $\mathrm{H}$ & 3.129409 & 1.237569 & -0.880088 \\
\hline $\mathrm{H}$ & 2.330468 & 2.551275 & -0.000965 \\
\hline $\mathrm{H}$ & 3.128605 & 1.238894 & 0.880926 \\
\hline C & 2.513662 & -1.470845 & 0.000027 \\
\hline $\mathrm{H}$ & 3.128736 & -1.23851 & -0.880744 \\
\hline $\mathrm{H}$ & 3.129325 & -1.237754 & 0.880174 \\
\hline $\mathrm{H}$ & 2.330546 & -2.551296 & 0.000501 \\
\hline
\end{tabular}

1,2,3,5-Tetramethylbenzene

\begin{tabular}{llll} 
Electronic Energy & \multicolumn{3}{c}{-389.3488489} \\
Electronic and Zero-point Energy & -389.1397797 & \\
Enthalpy & & -389.1277237 & \\
Free Energy & & -389.1761257 & \\
C & -0.380568 & -1.206838 & -0.003048 \\
C & -1.086304 & 0.013535 & -0.010833 \\
C & -0.366302 & 1.220848 & -0.004126 \\
C & 1.033423 & 1.18854 & -0.003043 \\
C & 1.744455 & -0.009536 & -0.003977 \\
C & 1.014623 & -1.200527 & -0.00127 \\
H & 1.548601 & -2.153516 & 0.004194 \\
C & -1.115955 & -2.514737 & 0.007674 \\
H & -1.808662 & -2.5887 & 0.857124 \\
H & -1.71932 & -2.654287 & -0.900237 \\
& & & S21
\end{tabular}




$\begin{array}{llll}\mathrm{H} & -0.418301 & -3.356924 & 0.072989 \\ \mathrm{C} & -2.587874 & -0.002163 & -0.005078 \\ \mathrm{H} & -2.988654 & -0.725118 & -0.726976 \\ \mathrm{H} & -2.985973 & -0.291699 & 0.978833 \\ \mathrm{H} & -3.017905 & 0.972724 & -0.252421 \\ \mathrm{C} & -1.063325 & 2.55182 & 0.00869 \\ \mathrm{H} & -1.684289 & 2.702134 & -0.884667 \\ \mathrm{H} & -1.725293 & 2.662384 & 0.877958 \\ \mathrm{H} & -0.335642 & 3.370117 & 0.042769 \\ \mathrm{H} & 1.581368 & 2.132851 & 0.000822 \\ \mathrm{C} & 3.243962 & -0.031373 & 0.001414 \\ \mathrm{H} & 3.635219 & -0.499384 & 0.914725 \\ \mathrm{H} & 3.640591 & -0.608234 & -0.844361 \\ \mathrm{H} & 3.661444 & 0.980242 & -0.059174\end{array}$

1,2,3,4-Tetramethylbenzene

\begin{tabular}{llll} 
Electronic Energy & \multicolumn{3}{l}{-389.3469981} \\
Electronic and Zero-point Energy & -389.137232 & \\
Enthalpy & & -389.125576 & \\
Free Energy & & -389.172452 & \\
C & 1.413104 & -0.577106 & -0.002219 \\
$\mathrm{C}$ & 0.704247 & 0.639952 & 0.012691 \\
$\mathrm{C}$ & -0.704265 & 0.639908 & -0.012913 \\
$\mathrm{C}$ & -1.413036 & -0.577188 & 0.002165 \\
$\mathrm{C}$ & -0.694965 & -1.773579 & 0.006833 \\
$\mathrm{C}$ & 0.695102 & -1.77354 & -0.006811 \\
$\mathrm{H}$ & 1.236063 & -2.72024 & -0.016682 \\
$\mathrm{C}$ & 2.914258 & -0.614761 & -0.021157 \\
$\mathrm{H}$ & 3.331801 & -0.037425 & -0.856905 \\
$\mathrm{H}$ & 3.353439 & -0.20374 & 0.898365 \\
$\mathrm{H}$ & 3.273846 & -1.6452 & -0.120583 \\
$\mathrm{C}$ & 1.451987 & 1.943812 & 0.044623 \\
$\mathrm{H}$ & 2.466095 & 1.824157 & 0.438198 \\
$\mathrm{H}$ & 1.549659 & 2.384633 & -0.959041 \\
$\mathrm{H}$ & 0.950632 & 2.689455 & 0.672085 \\
$\mathrm{C}$ & -2.914186 & -0.614885 & 0.021209 \\
$\mathrm{H}$ & -3.331713 & -0.037418 & 0.856867 \\
$\mathrm{H}$ & -3.353424 & -0.203998 & -0.898346 \\
$\mathrm{H}$ & -3.273738 & -1.645318 & 0.120819 \\
$\mathrm{H}$ & -1.235871 & -2.720307 & 0.016831 \\
$\mathrm{C}$ & -1.452183 & 1.943678 & -0.044545 \\
$\mathrm{H}$ & -1.550498 & 2.383982 & 0.959281 \\
& & & $\mathrm{~S} 22$ \\
& & & \\
& & & \\
& & & \\
& & & \\
& & &
\end{tabular}




$\begin{array}{llll}\mathrm{H} & -0.950627 & 2.689704 & -0.671373 \\ \mathrm{H} & -2.466046 & 1.82398 & -0.43877\end{array}$

\begin{tabular}{|c|c|c|c|}
\hline \multicolumn{4}{|c|}{ Pentamethylbenzene } \\
\hline \multicolumn{2}{|c|}{ Electronic Energy } & \multicolumn{2}{|c|}{-428.6450869} \\
\hline \multicolumn{2}{|c|}{ Electronic and Zero-point Energy } & \multicolumn{2}{|c|}{-428.4071792} \\
\hline \multicolumn{2}{|c|}{ Enthalpy } & \multicolumn{2}{|c|}{-428.3941412} \\
\hline \multicolumn{2}{|c|}{ Free Energy } & \multicolumn{2}{|c|}{-428.4438242} \\
\hline C & 1.22368 & -0.999953 & -0.003252 \\
\hline C & 1.225305 & 0.405433 & 0.004293 \\
\hline C & -0.000013 & 1.099782 & -0.016135 \\
\hline C & -1.225345 & 0.405352 & 0.004303 \\
\hline C & -1.223667 & -0.999968 & -0.003272 \\
\hline C & 0.000038 & -1.669785 & -0.013969 \\
\hline $\mathrm{H}$ & 0.000031 & -2.761674 & -0.025715 \\
\hline C & 2.497095 & -1.797864 & -0.004997 \\
\hline $\mathrm{H}$ & 3.137143 & -1.556208 & -0.864267 \\
\hline $\mathrm{H}$ & 3.098112 & -1.623253 & 0.897889 \\
\hline $\mathrm{H}$ & 2.280961 & -2.871401 & -0.048653 \\
\hline C & 2.519903 & 1.170246 & 0.037839 \\
\hline $\mathrm{H}$ & 3.372862 & 0.525065 & 0.266876 \\
\hline $\mathrm{H}$ & 2.736928 & 1.658167 & -0.923677 \\
\hline $\mathrm{H}$ & 2.50367 & 1.962594 & 0.797078 \\
\hline C & -0.000047 & 2.603333 & -0.048089 \\
\hline $\mathrm{H}$ & 0.00049 & 3.034869 & 0.96445 \\
\hline $\mathrm{H}$ & 0.879497 & 3.001917 & -0.563807 \\
\hline $\mathrm{H}$ & -0.88011 & 3.002019 & -0.562846 \\
\hline C & -2.497048 & -1.797939 & -0.005082 \\
\hline $\mathrm{H}$ & -3.136869 & -1.556535 & -0.864599 \\
\hline $\mathrm{H}$ & -2.280872 & -2.871482 & -0.048353 \\
\hline $\mathrm{H}$ & -3.098312 & -1.623091 & 0.897589 \\
\hline C & -2.519914 & 1.17023 & 0.037925 \\
\hline $\mathrm{H}$ & -2.503683 & 1.962302 & 0.79746 \\
\hline $\mathrm{H}$ & -2.736839 & 1.658509 & -0.923438 \\
\hline $\mathrm{H}$ & -3.372924 & 0.525007 & 0.266625 \\
\hline
\end{tabular}

Hexamethylbenzene

$\begin{array}{llll}\text { Electronic Energy } & -467.9377747 & \\ \text { Electronic and Zero-point Energy } & -467.6730143 & \\ \text { Enthalpy } & -467.6580323 & \\ \text { Free Energy } & -467.7125483 & \\ \text { C } & -0.187509 & -1.393613 & 0.012059\end{array}$




$\begin{array}{llll}\text { C } & -1.300399 & -0.534407 & -0.011304 \\ \mathrm{C} & -1.113096 & 0.859213 & 0.013024 \\ \mathrm{C} & 0.187518 & 1.393637 & -0.012178 \\ \mathrm{C} & 1.300419 & 0.534417 & 0.011213 \\ \mathrm{C} & 1.113093 & -0.859204 & -0.01318 \\ \mathrm{C} & -0.388598 & -2.884575 & 0.08872 \\ \mathrm{H} & -1.309232 & -3.14182 & 0.6226 \\ \mathrm{H} & -0.450143 & -3.355567 & -0.904245 \\ \mathrm{H} & 0.428999 & -3.376044 & 0.625954 \\ \mathrm{C} & -2.692005 & -1.105791 & -0.088909 \\ \mathrm{H} & -2.708885 & -2.058611 & -0.627866 \\ \mathrm{H} & -3.130882 & -1.290379 & 0.903509 \\ \mathrm{H} & -3.375134 & -0.435634 & -0.62077 \\ \mathrm{C} & -2.304463 & 1.778051 & 0.088534 \\ \mathrm{H} & -2.683681 & 2.061935 & -0.905097 \\ \mathrm{H} & -3.137576 & 1.316726 & 0.62884 \\ \mathrm{H} & -2.067549 & 2.706342 & 0.618508 \\ \mathrm{C} & 2.692066 & 1.105647 & 0.089179 \\ \mathrm{H} & 2.708958 & 2.058404 & 0.628227 \\ \mathrm{H} & 3.37499 & 0.435345 & 0.621124 \\ \mathrm{H} & 3.131155 & 1.290256 & -0.903123 \\ \mathrm{C} & 0.388477 & 2.884625 & -0.088757 \\ \mathrm{H} & -0.429048 & 3.375974 & -0.626224 \\ \mathrm{H} & 0.449658 & 3.355642 & 0.904216 \\ \mathrm{H} & 1.309212 & 3.141995 & -0.62238 \\ \mathrm{C} & 2.304521 & -1.777982 & -0.088569 \\ \mathrm{H} & 3.137691 & -1.316511 & -0.628658 \\ \mathrm{H} & 2.683559 & -2.061961 & 0.905106 \\ \mathrm{H} & 2.067761 & -2.706207 & -0.618716\end{array}$

benzene radical cation

\begin{tabular}{llll} 
Electronic Energy & \multicolumn{3}{c}{-231.8968633} \\
Electronic and Zero-point Energy & -231.7996792 & \\
Enthalpy & & -231.7935492 & \\
Free Energy & & -231.8283722 & \\
C & -1.233008 & 0.700945 & 0.000001 \\
C & 0.021356 & 1.379577 & -0.000002 \\
C & 1.25408 & 0.666137 & 0.00 \\
C & 1.233008 & -0.700945 & 0.00 \\
C & -0.021356 & -1.379577 & 0.000001 \\
C & -1.25408 & -0.666137 & 0.00 \\
H & -2.151257 & 1.280764 & 0.000002
\end{tabular}




$\begin{array}{llll}\mathrm{H} & 0.03522 & 2.467965 & -0.000003 \\ \mathrm{H} & 2.189942 & 1.216858 & 0.00 \\ \mathrm{H} & 2.151258 & -1.280764 & -0.000001 \\ \mathrm{H} & -0.035221 & -2.467965 & 0.000002 \\ \mathrm{H} & -2.189942 & -1.216859 & 0.000002\end{array}$

Toluene radical cation

Electronic Energy

Electronic and Zero-point Energy

Enthalpy

Free Energy

C

C

C

C

C

C

$\mathrm{H}$

$\mathrm{H}$

$\mathrm{H}$

$\mathrm{H}$

$\mathrm{H}$

C

$\mathrm{H}$

$\mathrm{H}$

$\mathrm{H}$
$-271.2107051$

$-271.085923$

$-271.07821$

$-271.117596$

$0.022971 \quad-0.000737$

$-1.227388-0.000106$

$-1.243774 \quad 0.000085$

$-0.017202 \quad-0.000027$

$1.228753 \quad 0.00006$

$1.24968 \quad 0.000034$

$-2.150747 \quad 0.000106$

$-2.180974 \quad 0.000324$

$\begin{array}{ll}-0.025036 & 0.000041\end{array}$

$2.15218 \quad 0.000136$

$2.18786 \quad 0.000367$

$0.008645-0.000386$

$-0.574144 \quad-0.860435$

$\begin{array}{ll}-0.546641 & 0.879795\end{array}$

$1.007392 \quad-0.013867$

o-Xylene radical cation

Electronic Energy

$-310.5183138$

Electronic and Zero-point Energy

$-310.365624$

Enthalpy

$-310.356733$

Free Energy

$-310.398186$

C

$-0.476824$

$-0.733939$

0.00249

C

$-0.476355$

0.734073

$-0.002634$

C

0.746583

1.41665

$-0.014644$

C

1.932482

0.716551

$-0.008934$

C

1.931988

$-0.717392$

0.009005

C

0.745597

$-1.416986$

0.014573

$\mathrm{H}$

0.748011

2.503898

$-0.023825$

$\mathrm{H}$

2.882982

1.244323

$-0.015019$

$\mathrm{H}$

2.882152

$-1.245785$

0.015181

$\mathrm{H}$

0.746743

$-2.504235$

0.023693 


$\begin{array}{llll}\mathrm{C} & -1.753893 & -1.468999 & -0.015642 \\ \mathrm{H} & -2.397023 & -1.16694 & 0.823722 \\ \mathrm{H} & -2.324897 & -1.224774 & -0.924775 \\ \mathrm{H} & -1.599631 & -2.549922 & 0.022558 \\ \mathrm{C} & -1.752883 & 1.469871 & 0.015669 \\ \mathrm{H} & -2.396892 & 1.167366 & -0.822872 \\ \mathrm{H} & -2.323391 & 1.226395 & 0.925369 \\ \mathrm{H} & -1.598219 & 2.550692 & -0.02333\end{array}$

$m$-Xylene radical cation

\begin{tabular}{llll} 
Electronic Energy & \multicolumn{3}{l}{-310.5184428} \\
Electronic and Zero-point Energy & -310.3667869 \\
Enthalpy & \multicolumn{3}{c}{-310.3574589} \\
Free Energy & \multicolumn{3}{c}{-310.4001689} \\
C & 1.206789 & -0.316345 & -0.00007 \\
C & 0.00 & -1.007212 & -0.000216 \\
C & -1.206804 & -0.316288 & -0.000086 \\
C & -1.191108 & 1.132722 & -0.000006 \\
C & -0.000013 & 1.839474 & 0.000005 \\
C & 1.191079 & 1.132711 & -0.000017 \\
H & -2.146515 & 1.655934 & 0.000065 \\
H & -0.000003 & 2.92554 & 0.000055 \\
H & 2.146499 & 1.655904 & 0.000021 \\
C & 2.512791 & -1.000294 & 0.000113 \\
H & 2.41584 & -2.088633 & -0.000097 \\
H & 3.102946 & -0.687501 & -0.874497 \\
H & 3.102482 & -0.687854 & 0.875167 \\
H & -0.000053 & -2.095752 & -0.000311 \\
$\mathrm{C}$ & -2.512754 & -1.000334 & 0.000118 \\
$\mathrm{H}$ & -2.415699 & -2.088664 & 0.00059 \\
$\mathrm{H}$ & -3.102676 & -0.687389 & 0.874831 \\
$\mathrm{H}$ & -3.102697 & -0.688184 & -0.874864
\end{tabular}

p-Xylene radical cation

Electronic Energy $\quad-310.5222826$

Electronic and Zero-point Energy $\quad-310.3700295$

Enthalpy $\quad-310.3606865$

Free Energy $\quad-310.4039245$

$\begin{array}{llll}\text { C } & -1.40644 & -0.018162 & -0.009439\end{array}$

C $\quad-0.673381 \quad-1.239602 \quad-0.005632$

$\begin{array}{llll}\text { C } & 0.69172 & -1.221614 & -0.000746\end{array}$

$\begin{array}{llll}\text { C } & 1.406508 & 0.020746 & 0.002218\end{array}$ 


$\begin{array}{llll}\mathrm{C} & 0.673635 & 1.24209 & -0.001441 \\ \mathrm{C} & -0.691942 & 1.224054 & -0.005907 \\ \mathrm{H} & 1.219492 & 2.182564 & -0.000303 \\ \mathrm{H} & -1.262227 & 2.151012 & -0.006167 \\ \mathrm{C} & -2.876461 & -0.010568 & 0.002916 \\ \mathrm{H} & -3.3057 & -1.002505 & -0.157381 \\ \mathrm{H} & -3.22723 & 0.368782 & 0.977604 \\ \mathrm{H} & -3.271177 & 0.698664 & -0.737611 \\ \mathrm{H} & -1.220179 & -2.179621 & -0.007077 \\ \mathrm{C} & 2.87665 & 0.007367 & 0.005714 \\ \mathrm{H} & 3.248116 & -0.594709 & 0.848721 \\ \mathrm{H} & 3.244742 & -0.509945 & -0.894676 \\ \mathrm{H} & 3.310502 & 1.008508 & 0.050234 \\ \mathrm{H} & 1.261932 & -2.148609 & 0.000565\end{array}$

mesitylene radical cation

\begin{tabular}{llll} 
Electronic Energy & \multicolumn{3}{l}{-349.8212729} \\
Electronic and Zero-point Energy & -349.6424149 \\
Enthalpy & \multicolumn{3}{c}{-349.6314569} \\
Free Energy & & -349.6775039 \\
C & -1.324502 & -0.528685 & 0.000065 \\
$\mathrm{C}$ & -0.17205 & -1.347158 & 0.000267 \\
$\mathrm{C}$ & 1.168313 & -0.814154 & -0.004596 \\
$\mathrm{C}$ & 1.329241 & 0.553148 & -0.007839 \\
$\mathrm{C}$ & 0.20212 & 1.396272 & -0.001958 \\
$\mathrm{C}$ & -1.122622 & 0.831322 & 0.000047 \\
$\mathrm{H}$ & -1.97038 & 1.516405 & 0.00178 \\
$\mathrm{C}$ & -2.679816 & -1.151502 & -0.000202 \\
$\mathrm{H}$ & -2.814311 & -1.786975 & -0.884177 \\
$\mathrm{H}$ & -2.812001 & -1.794349 & 0.878729 \\
$\mathrm{H}$ & -3.469604 & -0.394702 & 0.00374 \\
$\mathrm{H}$ & -0.29297 & -2.431987 & 0.004111 \\
$\mathrm{H}$ & 2.325117 & 0.991707 & -0.011956 \\
$\mathrm{C}$ & 2.304422 & -1.762252 & 0.002987 \\
$\mathrm{H}$ & 2.28751 & -2.364577 & 0.922699 \\
$\mathrm{H}$ & 2.217227 & -2.477318 & -0.826555 \\
$\mathrm{H}$ & 3.267759 & -1.250094 & -0.064276 \\
$\mathrm{C}$ & 0.336621 & 2.861228 & 0.004075 \\
$\mathrm{H}$ & -0.186524 & 3.289969 & -0.864383 \\
$\mathrm{H}$ & -0.179739 & 3.280647 & 0.881219 \\
$\mathrm{H}$ & 1.377553 & 3.191952 & 0.001986
\end{tabular}


1,2,3-trimethylbenzene radical cation

\begin{tabular}{llll} 
Electronic Energy & \multicolumn{3}{l}{-349.8201942} \\
Electronic and Zero-point Energy & -349.640752 & \\
Enthalpy & & -349.629857 & \\
Free Energy & & -349.676713 & \\
$\mathrm{C}$ & 1.253199 & 0.086419 & 0.003227 \\
$\mathrm{C}$ & 0.008045 & -0.66315 & -0.0029 \\
$\mathrm{C}$ & -1.267913 & 0.030148 & 0.002798 \\
$\mathrm{C}$ & -1.264639 & 1.405 & -0.00349 \\
$\mathrm{C}$ & -0.050508 & 2.119842 & -0.008384 \\
$\mathrm{C}$ & 1.194007 & 1.458906 & -0.003154 \\
$\mathrm{H}$ & 2.112146 & 2.041669 & -0.003057 \\
$\mathrm{C}$ & 2.555693 & -0.628261 & 0.014103 \\
$\mathrm{H}$ & 2.65155 & -1.270296 & 0.899869 \\
$\mathrm{H}$ & 2.657792 & -1.285819 & -0.859305 \\
$\mathrm{H}$ & 3.387506 & 0.080962 & 0.011102 \\
$\mathrm{H}$ & -0.074327 & 3.208044 & -0.012846 \\
$\mathrm{C}$ & 0.071759 & -2.131417 & -0.018799 \\
$\mathrm{H}$ & 0.647909 & -2.475101 & -0.892443 \\
$\mathrm{H}$ & 0.641256 & -2.494239 & 0.851447 \\
$\mathrm{H}$ & -0.90441 & -2.615847 & -0.027382 \\
$\mathrm{H}$ & -2.205756 & 1.949469 & -0.00306 \\
$\mathrm{C}$ & -2.54435 & -0.731627 & 0.014146 \\
$\mathrm{H}$ & -2.636397 & -1.373215 & -0.872506 \\
$\mathrm{H}$ & -2.610479 & -1.391197 & 0.889728 \\
$\mathrm{H}$ & -3.398549 & -0.049595 & 0.033169
\end{tabular}

1,2,4-trimethylbenzene radical cation

\begin{tabular}{llll} 
Electronic Energy & \multicolumn{3}{c}{-349.8282736} \\
Electronic and Zero-point Energy & -349.6479835 & \\
Enthalpy & & -349.6373205 & \\
Free Energy & & -349.6833455 & \\
C & 1.111868 & -0.611717 & -0.00039 \\
C & 0.699089 & 0.78555 & -0.004119 \\
C & -0.649293 & 1.083935 & -0.0059 \\
C & -1.621763 & 0.073315 & -0.002087 \\
C & -1.201021 & -1.303891 & -0.000398 \\
C & 0.127519 & -1.626533 & 0.00008 \\
H & 0.44488 & -2.666479 & 0.001504 \\
C & 2.543022 & -0.961359 & 0.001197 \\
H & 3.043985 & -0.548028 & -0.887454 \\
H & 3.055954 & -0.505783 & 0.861337 \\
& & & S28
\end{tabular}




$\begin{array}{llll}\mathrm{H} & 2.700754 & -2.042178 & 0.023954 \\ \mathrm{H} & -0.96988 & 2.124243 & -0.008015 \\ \mathrm{H} & -1.963555 & -2.081205 & 0.000907 \\ \mathrm{C} & 1.724523 & 1.855468 & 0.002633 \\ \mathrm{H} & 2.342526 & 1.795984 & 0.909472 \\ \mathrm{H} & 2.414985 & 1.750348 & -0.844917 \\ \mathrm{H} & 1.267319 & 2.847133 & -0.040155 \\ \mathrm{C} & -3.063276 & 0.378874 & 0.003021 \\ \mathrm{H} & -3.267919 & 1.452091 & -0.010431 \\ \mathrm{H} & -3.554081 & -0.097877 & -0.858683 \\ \mathrm{H} & -3.538984 & -0.070101 & 0.888256\end{array}$

1,2,4,5-tetramethylbenzene radical cation

\begin{tabular}{llll} 
Electronic Energy & \multicolumn{3}{c}{-389.1351373} \\
Electronic and Zero-point Energy & -388.9267261 \\
Enthalpy & & -388.9149471 \\
Free Energy & & -388.9625751 \\
$\mathrm{C}$ & 1.215177 & 0.728803 & -0.009992 \\
$\mathrm{C}$ & 1.215152 & -0.728809 & 0.009943 \\
$\mathrm{C}$ & -0.00003 & -1.403347 & -0.000001 \\
$\mathrm{C}$ & -1.215188 & -0.728783 & -0.009965 \\
$\mathrm{C}$ & -1.215162 & 0.728796 & 0.009865 \\
$\mathrm{C}$ & 0.000019 & 1.403352 & -0.000134 \\
$\mathrm{H}$ & 0.000004 & 2.492228 & -0.000234 \\
$\mathrm{C}$ & 2.495677 & 1.468404 & -0.042235 \\
$\mathrm{H}$ & 3.129024 & 1.200366 & 0.815061 \\
$\mathrm{H}$ & 3.075123 & 1.202086 & -0.938363 \\
$\mathrm{H}$ & 2.336316 & 2.549707 & -0.038131 \\
$\mathrm{C}$ & 2.495621 & -1.468452 & 0.042307 \\
$\mathrm{H}$ & 3.129192 & -1.200256 & -0.814762 \\
$\mathrm{H}$ & 3.074821 & -1.202372 & 0.938665 \\
$\mathrm{H}$ & 2.336217 & -2.549751 & 0.037962 \\
$\mathrm{H}$ & -0.00004 & -2.492224 & 0.000015 \\
$\mathrm{C}$ & -2.495682 & -1.46842 & -0.042298 \\
$\mathrm{H}$ & -3.075082 & -1.202093 & -0.93844 \\
$\mathrm{H}$ & -2.336248 & -2.549717 & -0.038324 \\
$\mathrm{H}$ & -3.129008 & -1.200539 & 0.81506 \\
$\mathrm{C}$ & -2.495609 & 1.468474 & 0.042354 \\
$\mathrm{H}$ & -3.129584 & 1.200009 & -0.814309 \\
$\mathrm{H}$ & -3.074407 & 1.202695 & 0.939087 \\
$\mathrm{H}$ & -2.336175 & 2.549762 & 0.037653
\end{tabular}




\begin{tabular}{llll} 
1,2,3,5-tetramethylbenzene radical cation \\
Electronic Energy & \multicolumn{3}{c}{-389.1300642} \\
Electronic and Zero-point Energy & -388.9224262 \\
Enthalpy & & -388.9101622 \\
Free Energy & & -388.9599962 \\
C & -0.373023 & -1.243092 & -0.013716 \\
C & -1.077397 & 0.019977 & -0.003676 \\
C & -0.339399 & 1.265455 & -0.014103 \\
C & 1.032642 & 1.218203 & -0.015398 \\
C & 1.73056 & -0.014667 & -0.01124 \\
$\mathrm{C}$ & 0.997386 & -1.23285 & -0.013603 \\
$\mathrm{H}$ & 1.548231 & -2.172587 & -0.014437 \\
$\mathrm{C}$ & -1.129957 & -2.525829 & -0.023106 \\
$\mathrm{H}$ & -1.818153 & -2.594865 & 0.829004 \\
$\mathrm{H}$ & -1.741175 & -2.62276 & -0.930296 \\
$\mathrm{H}$ & -0.446139 & -3.37798 & 0.018637 \\
$\mathrm{C}$ & -2.550008 & 0.011508 & 0.048215 \\
$\mathrm{H}$ & -2.966001 & -0.669769 & -0.706326 \\
$\mathrm{H}$ & -2.881002 & -0.397771 & 1.017259 \\
$\mathrm{H}$ & -3.000624 & 0.996837 & -0.073854 \\
$\mathrm{C}$ & -1.052832 & 2.571906 & -0.021295 \\
$\mathrm{H}$ & -1.693762 & 2.672605 & -0.907082 \\
$\mathrm{H}$ & -1.705 & 2.678468 & 0.85546 \\
$\mathrm{H}$ & -0.339282 & 3.40034 & -0.020201 \\
$\mathrm{H}$ & 1.606641 & 2.143608 & -0.018389 \\
$\mathrm{C}$ & 3.20416 & -0.045655 & 0.025924 \\
$\mathrm{H}$ & 3.529199 & -0.233042 & 1.063113 \\
$\mathrm{H}$ & 3.604649 & -0.874407 & -0.56945 \\
$\mathrm{H}$ & 3.649631 & 0.901583 & -0.291447
\end{tabular}

1,2,3,4-tetramethylbenzene radical cation

\begin{tabular}{llll} 
Electronic Energy & \multicolumn{3}{c}{-389.1269023} \\
Electronic and Zero-point Energy & -388.9186971 & \\
Enthalpy & & -388.9067561 & \\
Free Energy & & -388.9547451 & \\
C & 1.400056 & -0.578028 & -0.00658 \\
C & 0.692414 & 0.672554 & 0.029503 \\
C & -0.692318 & 0.672559 & -0.029488 \\
C & -1.400078 & -0.57806 & 0.006592 \\
C & -0.68009 & -1.811894 & 0.008246 \\
C & 0.67998 & -1.81188 & -0.008366 \\
H & 1.241369 & -2.743292 & -0.021953 \\
& & & S30
\end{tabular}




$\begin{array}{llll}\mathrm{C} & 2.874235 & -0.616341 & -0.052734 \\ \mathrm{H} & 3.272187 & 0.066832 & -0.815374 \\ \mathrm{H} & 3.293083 & -0.26671 & 0.904706 \\ \mathrm{H} & 3.248693 & -1.626143 & -0.237483 \\ \mathrm{C} & 1.464928 & 1.948839 & 0.097103 \\ \mathrm{H} & 2.422653 & 1.819066 & 0.608496 \\ \mathrm{H} & 1.684337 & 2.330375 & -0.910989 \\ \mathrm{H} & 0.911302 & 2.728728 & 0.627484 \\ \mathrm{C} & -2.874248 & -0.616191 & 0.052882 \\ \mathrm{H} & -3.272082 & 0.067162 & 0.815412 \\ \mathrm{H} & -3.293118 & -0.266728 & -0.904619 \\ \mathrm{H} & -3.248784 & -1.625938 & 0.237785 \\ \mathrm{H} & -1.241466 & -2.743314 & 0.021782 \\ \mathrm{C} & -1.464868 & 1.948783 & -0.097168 \\ \mathrm{H} & -1.684787 & 2.330058 & 0.910916 \\ \mathrm{H} & -0.911081 & 2.728845 & -0.627118 \\ \mathrm{H} & -2.422373 & 1.819011 & -0.608981\end{array}$

pentamethylbenzene radical cation

\begin{tabular}{llll} 
Electronic Energy & \multicolumn{3}{l}{-428.4312422} \\
Electronic and Zero-point Energy & -428.195578 & \\
Enthalpy & & -428.182116 & \\
Free Energy & & -428.233611 & \\
$\mathrm{C}$ & 1.208515 & -1.0297 & 0.002488 \\
$\mathrm{C}$ & 1.210136 & 0.427201 & -0.025692 \\
$\mathrm{C}$ & 0.000429 & 1.133662 & 0.034854 \\
$\mathrm{C}$ & -1.209754 & 0.427482 & -0.025752 \\
$\mathrm{C}$ & -1.208995 & -1.029494 & 0.002795 \\
$\mathrm{C}$ & -0.000502 & -1.710062 & 0.020442 \\
$\mathrm{H}$ & -0.000469 & -2.797853 & 0.057287 \\
$\mathrm{C}$ & 2.484586 & -1.781137 & 0.026966 \\
$\mathrm{H}$ & 3.028617 & -1.653842 & -0.920824 \\
$\mathrm{H}$ & 3.154883 & -1.413594 & 0.815445 \\
$\mathrm{H}$ & 2.312254 & -2.849916 & 0.179107 \\
$\mathrm{C}$ & 2.50708 & 1.1449 & -0.101189 \\
$\mathrm{H}$ & 2.862881 & 1.387928 & 0.91348 \\
$\mathrm{H}$ & 3.288034 & 0.546722 & -0.576773 \\
$\mathrm{H}$ & 2.418352 & 2.092128 & -0.640771 \\
$\mathrm{C}$ & 0.000366 & 2.630582 & 0.128386 \\
$\mathrm{H}$ & 0.879322 & 2.997004 & 0.666249 \\
$\mathrm{H}$ & 0.000007 & 3.103715 & -0.863207 \\
$\mathrm{H}$ & -0.878155 & 2.996935 & 0.666999
\end{tabular}




$\begin{array}{llll}\mathrm{C} & -2.485636 & -1.780015 & 0.027716 \\ \mathrm{H} & -3.029505 & -1.653176 & -0.920236 \\ \mathrm{H} & -2.313936 & -2.8488 & 0.180596 \\ \mathrm{H} & -3.15579 & -1.411528 & 0.815829 \\ \mathrm{C} & -2.506246 & 1.145707 & -0.101945 \\ \mathrm{H} & -2.862863 & 1.38896 & 0.912322 \\ \mathrm{H} & -2.416595 & 2.09286 & -0.641579 \\ \mathrm{H} & -3.286911 & 0.547704 & -0.57833\end{array}$

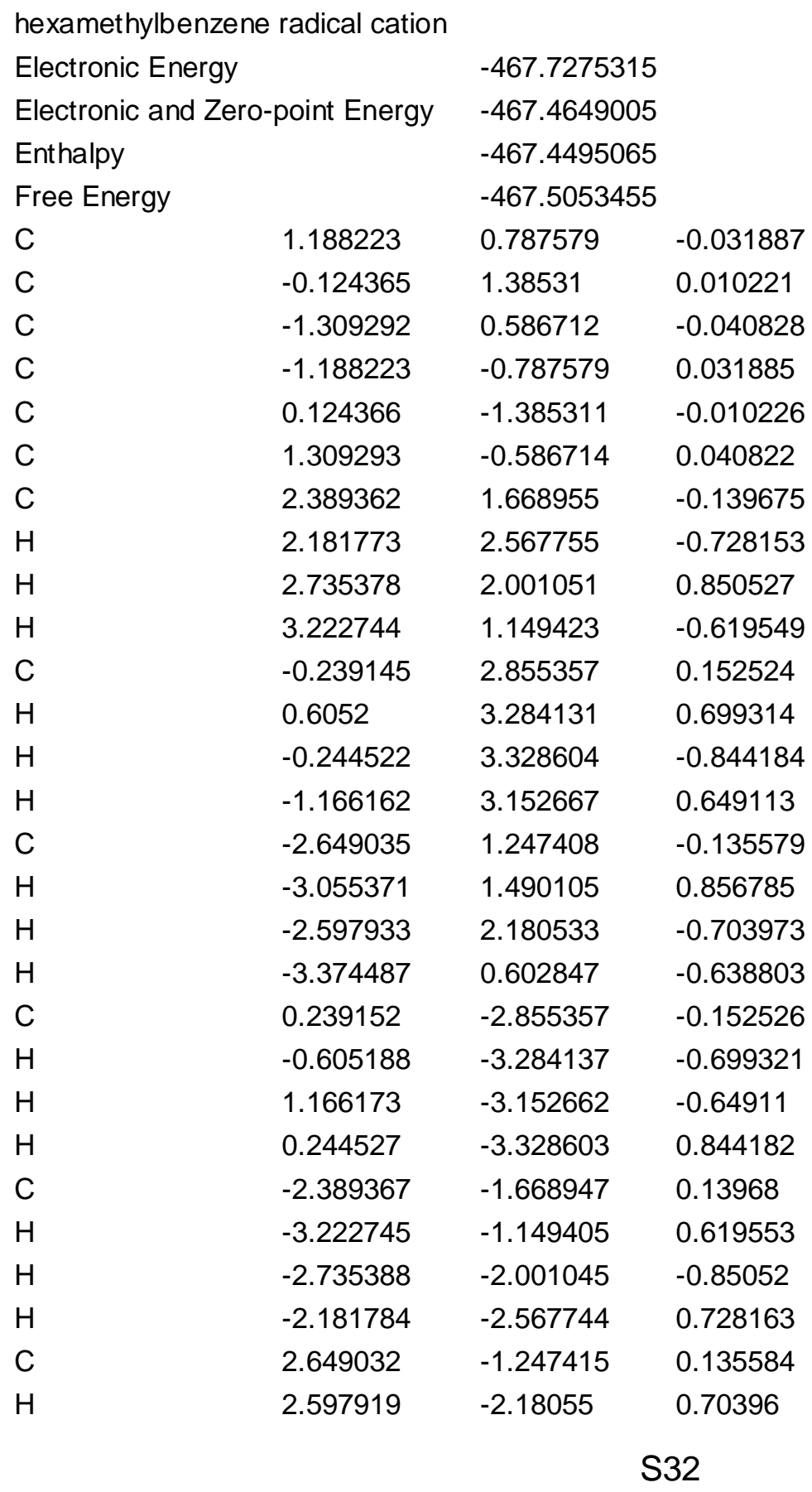




$\begin{array}{llll}\mathrm{H} & 3.055383 & -1.490096 & -0.856778 \\ \mathrm{H} & 3.374479 & -0.602865 & 0.638831\end{array}$

$\mathrm{Tl}(\mathrm{TFA})_{3}$-benzene $\mathrm{C}-\mathrm{H}$ activation transition state

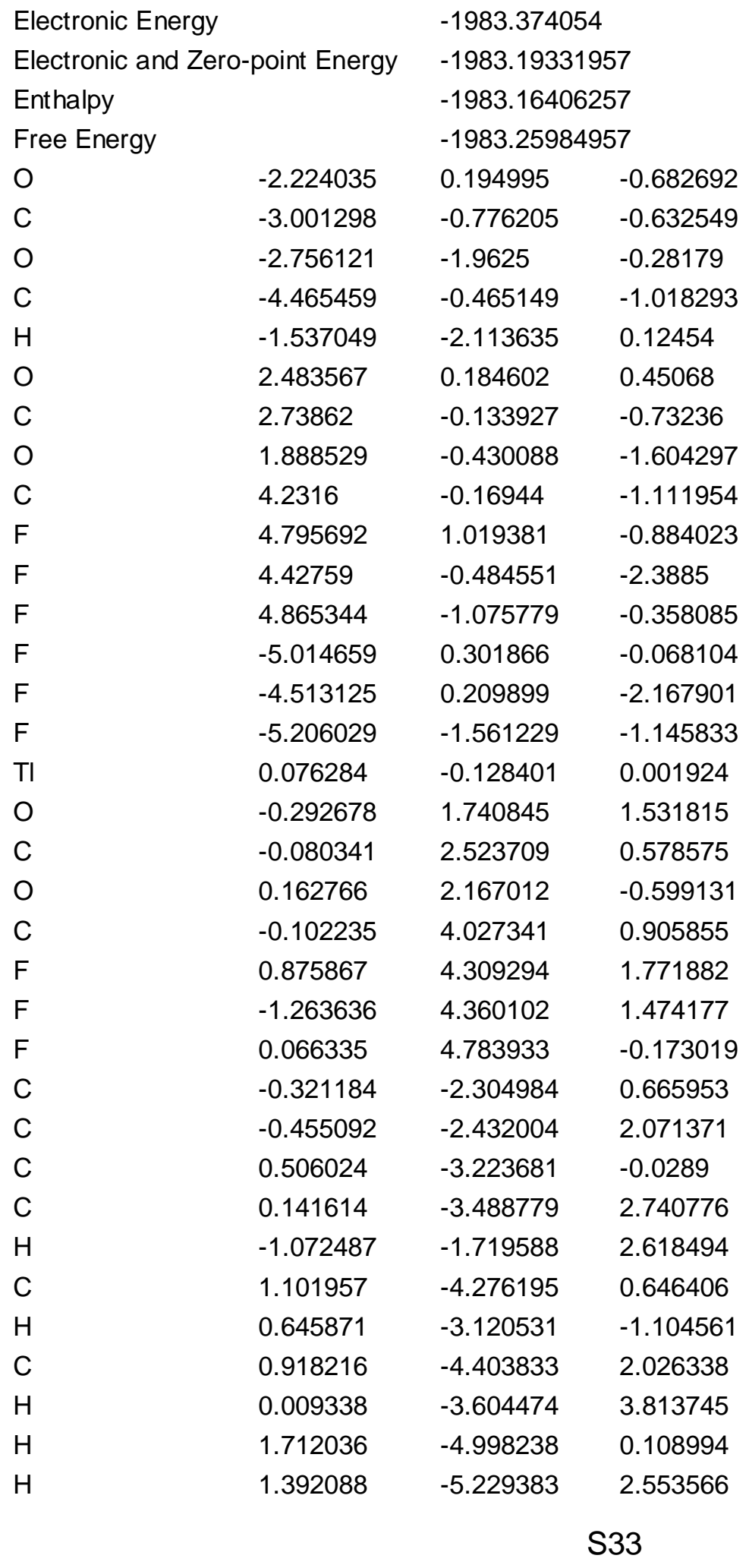




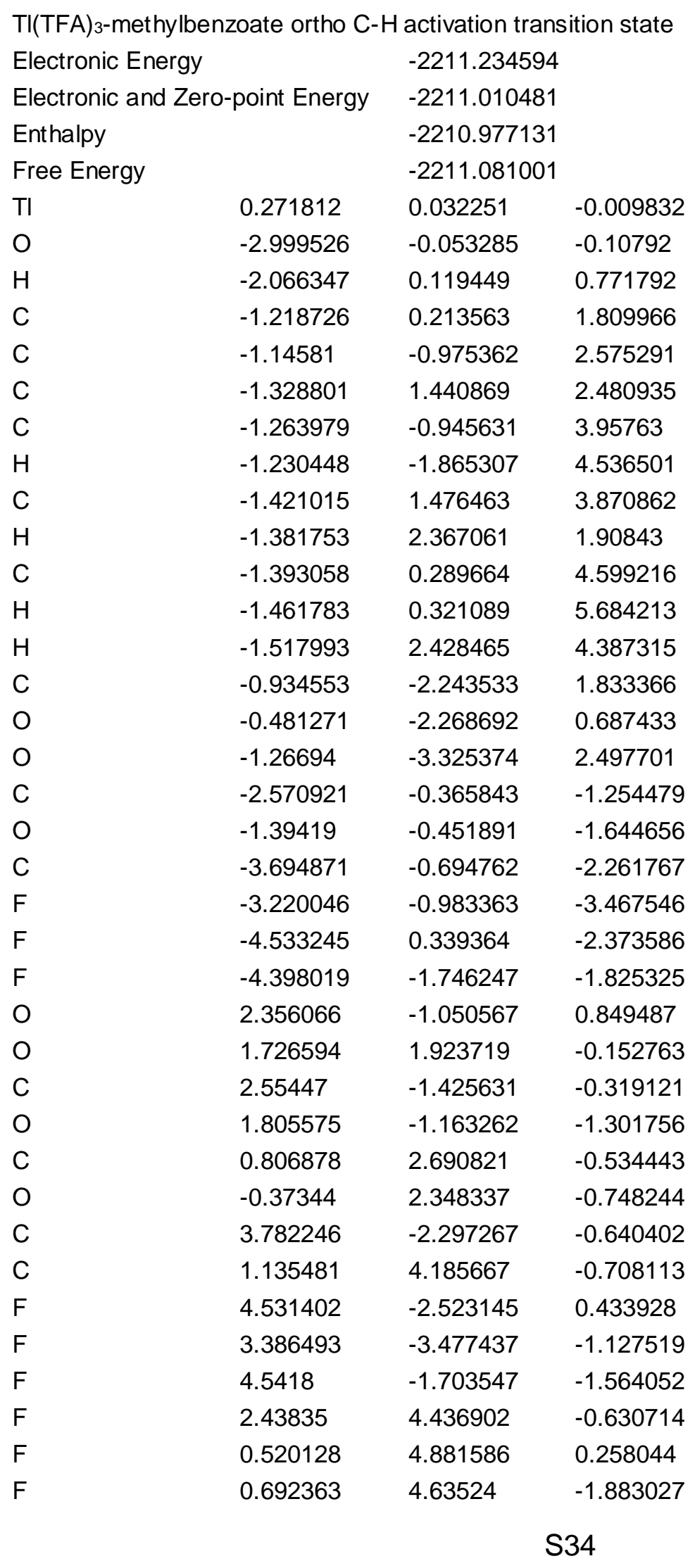




$\begin{array}{llll}\mathrm{C} & -1.054132 & -4.589441 & 1.839461 \\ \mathrm{H} & -1.641224 & -4.634979 & 0.918236 \\ \mathrm{H} & 0.00873 & -4.719027 & 1.616911 \\ \mathrm{H} & -1.394033 & -5.344756 & 2.547814\end{array}$

TI(TFA)3-methylbenzoate meta $\mathrm{C}-\mathrm{H}$ activation transition state

Electronic Energy

Electronic and Zero-point Energy

Enthalpy

Free Energy

$\mathrm{TI}$

O

$\mathrm{H}$

C

C

C

C

C

$\mathrm{H}$

C

$\mathrm{H}$

$\mathrm{H}$

C

O

C

$\mathrm{F}$

$\mathrm{F}$

$\mathrm{F}$

$\mathrm{O}$

O

C

O

C

O

C

C

F

F

$\mathrm{F}$

$\mathrm{F}$

F

F
$-2211.222986$

$-2210.999346$

$-2210.965676$

$-2211.070656$

$\begin{array}{lll}0.083407 & 0.397154 & -0.065262\end{array}$

$0.488972 \quad-2.796767 \quad-0.936786$

$\begin{array}{lll}1.025266 & -1.947711 & -0.102709\end{array}$

$\begin{array}{lll}1.595649 & -1.150162 & 0.796879\end{array}$

$\begin{array}{lll}2.955058 & -0.868942 & 0.525627\end{array}$

$\begin{array}{lll}1.222065 & -1.51869 & 2.113996\end{array}$

$\begin{array}{lll}3.911237 & -1.019985 & 1.522816\end{array}$

$\begin{array}{lll}2.179588 & -1.668812 & 3.104792\end{array}$

$\begin{array}{lll}0.174086 & -1.723902 & 2.336173\end{array}$

$\begin{array}{lll}3.51655 & -1.417473 & 2.80739\end{array}$

$\begin{array}{lll}4.277462 & -1.526307 & 3.577894\end{array}$

$1.893304 \quad-1.977517 \quad 4.106735$

$\begin{array}{lll}-0.194504 & -2.222502 & -1.829201\end{array}$

$\begin{array}{lll}-0.448995 & -1.010943 & -1.945391\end{array}$

$\begin{array}{lll}-0.770726 & -3.186456 & -2.891227\end{array}$

$\begin{array}{lll}-1.494436 & -2.54979 & -3.804691\end{array}$

$\begin{array}{lll}-1.544273 & -4.104661 & -2.30529\end{array}$

$0.223017 \quad-3.828006 \quad-3.516194$

$\begin{array}{lll}0.527901 & 2.614143 & -0.763647\end{array}$

$\begin{array}{lll}-1.242324 & 1.211159 & 1.792707\end{array}$

$\begin{array}{lll}-0.61675 & 2.722912 & -1.281448\end{array}$

$\begin{array}{lll}-1.465301 & 1.812143 & -1.310794\end{array}$

$\begin{array}{lll}-2.013371 & 0.224619 & 1.718656\end{array}$

$\begin{array}{lll}-1.910226 & -0.710885 & 0.894721\end{array}$

$\begin{array}{lll}-0.94584 & 4.095001 & -1.895861\end{array}$

$\begin{array}{lll}-3.170994 & 0.123856 & 2.730378\end{array}$

$\begin{array}{lll}-2.136598 & 4.110362 & -2.481565\end{array}$

$\begin{array}{lll}-0.933671 & 5.030716 & -0.94272\end{array}$

$\begin{array}{lll}-0.023965 & 4.420978 & -2.805572\end{array}$

$\begin{array}{lll}-2.982307 & -0.945147 & 3.515049\end{array}$

$\begin{array}{lll}-4.334424 & -0.033102 & 2.096766\end{array}$

$\begin{array}{lll}-3.261108 & 1.196945 & 3.509852\end{array}$ 


$\begin{array}{llll}\mathrm{H} & 3.259776 & -0.570514 & -0.475607 \\ \mathrm{C} & 5.356664 & -0.77571 & 1.279463 \\ \mathrm{O} & 6.21612 & -0.909547 & 2.131558 \\ \mathrm{O} & 5.611255 & -0.395274 & 0.029824 \\ \mathrm{C} & 6.989801 & -0.158734 & -0.29142 \\ \mathrm{H} & 7.573996 & -1.071268 & -0.13863 \\ \mathrm{H} & 7.002075 & 0.129709 & -1.34295 \\ \mathrm{H} & 7.390829 & 0.647292 & 0.330561\end{array}$

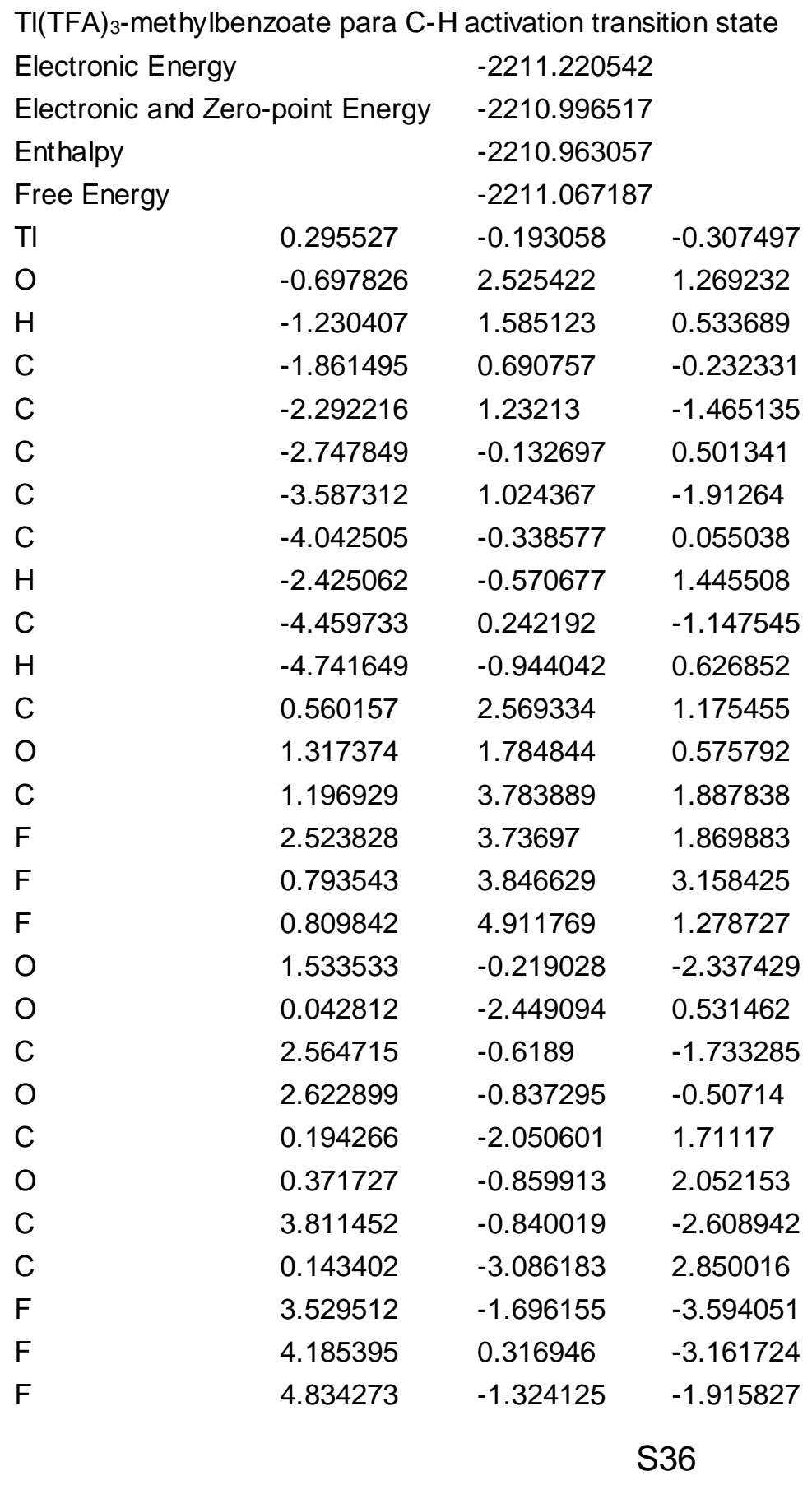




$\begin{array}{llll}\mathrm{F} & 0.034826 & -4.332289 & 2.401011 \\ \mathrm{~F} & -0.91012 & -2.832672 & 3.63574 \\ \mathrm{~F} & 1.244696 & -3.004187 & 3.598486 \\ \mathrm{H} & -1.615343 & 1.853112 & -2.050241 \\ \mathrm{H} & -3.923636 & 1.465258 & -2.846865 \\ \mathrm{C} & -5.867257 & -0.002473 & -1.579467 \\ \mathrm{O} & -6.644905 & -0.708555 & -0.96531 \\ \mathrm{O} & -6.177338 & 0.636776 & -2.702 \\ \mathrm{C} & -7.519387 & 0.464098 & -3.179816 \\ \mathrm{H} & -8.233105 & 0.844163 & -2.442526 \\ \mathrm{H} & -7.580392 & 1.042986 & -4.102013 \\ \mathrm{H} & -7.718296 & -0.592977 & -3.379678\end{array}$

$\mathrm{TI}(\mathrm{TFA})_{3}$-toluene ortho $\mathrm{C}-\mathrm{H}$ activation transition state

Electronic Energy

Electronic and Zero-point Energy

Enthalpy

Free Energy

O

C

O

C

$\mathrm{H}$

O

C

O

C

$\mathrm{F}$

$\mathrm{F}$

$\mathrm{F}$

F

$\mathrm{F}$

$\mathrm{F}$

TI

O

C

O

C

$\mathrm{F}$

$\mathrm{F}$

$\mathrm{F}$

C
$-2022.68196884$

$-2022.47328389$

$-2022.44272389$

$-2022.53942889$

$\begin{array}{ll}-0.073611 & 0.33368\end{array}$

$-0.809235 \quad 0.016582$

$-1.826542 \quad-0.728307$

$-0.398868 \quad 0.601741$

$-2.021166 \quad-1.266484$

$-0.413437 \quad-0.773641$

$-1.132348 \quad 0.2472$

$-1.598876 \quad 0.883526$

$-1.464133 \quad 0.735783$

$-0.341654 \quad 0.938293$

$-2.164373 \quad 1.865886$

$-2.18017 \quad-0.197191$

$0.817789 \quad 0.155544$

$-0.340466 \quad 1.934746$

$-1.241581 \quad 0.270459$

$-0.522764 \quad-0.615084$

$1.778786-1.407087$

$2.175245-0.249422$

$1.425882 \quad 0.73179$

$3.699847 \quad-0.05437$

$4.201715-0.81336$

$4.267911-0.435835$

$4.041493 \quad 1.207778$

$-2.184184-1.956814$ 


$\begin{array}{llll}\mathrm{C} & 1.107711 & -1.770256 & -3.295292 \\ \mathrm{C} & 0.218585 & -3.436292 & -1.711961 \\ \mathrm{C} & 0.831026 & -2.662152 & -4.330833 \\ \mathrm{C} & -0.061298 & -4.294203 & -2.756885 \\ \mathrm{H} & -0.004961 & -3.732574 & -0.68719 \\ \mathrm{C} & 0.248312 & -3.89759 & -4.064404 \\ \mathrm{H} & 1.063678 & -2.380821 & -5.356811 \\ \mathrm{H} & -0.515449 & -5.264374 & -2.571548 \\ \mathrm{H} & 0.026074 & -4.568192 & -4.892407 \\ \mathrm{C} & 1.698087 & -0.429576 & -3.578914 \\ \mathrm{H} & 0.915354 & 0.27704 & -3.888786 \\ \mathrm{H} & 2.189173 & -0.004149 & -2.693276 \\ \mathrm{H} & 2.428653 & -0.477241 & -4.393835\end{array}$

TI(TFA)3-toluene meta $\mathrm{C}-\mathrm{H}$ activation transition state

Electronic Energy

Electronic and Zero-point Energy

Enthalpy

Free Energy

O

C

O

C

$\mathrm{H}$

O

C

O

C

$\mathrm{F}$

$\mathrm{F}$

$\mathrm{F}$

$\mathrm{F}$

$\mathrm{F}$

$\mathrm{F}$

$\mathrm{TI}$

O

C

$\mathrm{O}$

C

$\mathrm{F}$

$\mathrm{F}$

$\mathrm{F}$
$-2022.67617263$

$-2022.46788856$

$-2022.43695856$

$-2022.53548056$

$0.053777 \quad 0.181312$

$-0.722688 \quad-0.061741$

$-1.809033 \quad-0.701961$

$-0.289309 \quad 0.502742$

$-2.043896 \quad-1.197934$

$-0.412779-0.852173$

$-1.132966 \quad 0.168314$

$-1.577272 \quad 0.833334$

$-1.503326 \quad 0.603524$

$-0.404469 \quad 0.753453$

$-2.183058 \quad 1.74566$

$-2.258751 \quad-0.342995$

$0.915009 \quad 0.025258$

$-0.197036 \quad 1.834481$

$-1.139833 \quad 0.193191$

$-0.473875 \quad-0.661447$

$1.839696-1.345709$

$2.191215-0.168715$

$1.406975 \quad 0.794879$

$3.701882 \quad 0.061972$

$4.119808-0.583449$

$4.376362-0.421877$

$4.006034 \quad 1.348206$ 


$\begin{array}{llll}\mathrm{C} & 0.876118 & -2.224721 & -1.904767 \\ \mathrm{C} & 1.150308 & -1.8521 & -3.246293 \\ \mathrm{C} & 0.164829 & -3.423041 & -1.655366 \\ \mathrm{C} & 0.808294 & -2.675218 & -4.312211 \\ \mathrm{C} & -0.174852 & -4.252696 & -2.712715 \\ \mathrm{H} & -0.084444 & -3.70811 & -0.634036 \\ \mathrm{C} & 0.142035 & -3.875887 & -4.018079 \\ \mathrm{H} & -0.692831 & -5.191481 & -2.530468 \\ \mathrm{H} & -0.137887 & -4.532416 & -4.842341 \\ \mathrm{C} & 1.124613 & -2.309919 & -5.728252 \\ \mathrm{H} & 1.745802 & -3.077541 & -6.205775 \\ \mathrm{H} & 0.208113 & -2.221158 & -6.324919 \\ \mathrm{H} & 1.660007 & -1.356677 & -5.786432 \\ \mathrm{H} & 1.687992 & -0.921648 & -3.440023\end{array}$

TI(TFA) 3 -toluene para C-H activation transition state

\begin{tabular}{llll} 
Electronic Energy & & \multicolumn{2}{l}{-2022.67921471} \\
Electronic and Zero-point Energy & -2022.47115628 \\
Enthalpy & & -2022.44011328 \\
Free Energy & & -2022.53997828 \\
O & 2.276611 & 0.069639 & 0.104308 \\
C & 3.192517 & -0.743085 & -0.107025 \\
O & 3.136529 & -1.855483 & -0.70095 \\
C & 4.581391 & -0.32632 & 0.428138 \\
H & 1.965396 & -2.071413 & -1.198242 \\
O & -2.441401 & -0.362704 & -0.851095 \\
C & -2.541685 & -1.094318 & 0.161438 \\
O & -1.58412 & -1.560436 & 0.817858 \\
C & -3.976503 & -1.449696 & 0.596106 \\
F & -4.705877 & -0.343253 & 0.756573 \\
F & -4.009713 & -2.138461 & 1.732846 \\
F & -4.5599 & -2.190558 & -0.355272 \\
F & 4.959155 & 0.822157 & -0.143719 \\
F & 4.520929 & -0.125701 & 1.747828 \\
F & 5.519025 & -1.237865 & 0.188284 \\
TI & 0.010441 & -0.451207 & -0.682601 \\
O & 0.000614 & 1.881801 & -1.306793 \\
C & -0.230882 & 2.204109 & -0.115728 \\
O & -0.354828 & 1.396414 & 0.83 \\
C & -0.392319 & 3.71022 & 0.156537 \\
F & -1.482817 & 4.161123 & -0.472854 \\
F & 0.661096 & 4.381593 & -0.314489
\end{tabular}




$\begin{array}{llll}\text { F } & -0.515927 & 3.982325 & 1.450886 \\ \mathrm{C} & 0.83514 & -2.216256 & -1.905706 \\ \mathrm{C} & 1.136738 & -1.862691 & -3.249077 \\ \mathrm{C} & 0.101827 & -3.409551 & -1.675628 \\ \mathrm{C} & 0.807404 & -2.706767 & -4.290923 \\ \mathrm{H} & 1.682087 & -0.939965 & -3.450142 \\ \mathrm{C} & -0.222479 & -4.247541 & -2.725296 \\ \mathrm{H} & -0.179082 & -3.684616 & -0.659423 \\ \mathrm{C} & 0.123762 & -3.910227 & -4.045143 \\ \mathrm{H} & 1.073399 & -2.44539 & -5.314204 \\ \mathrm{H} & -0.758136 & -5.176503 & -2.535956 \\ \mathrm{C} & -0.207238 & -4.82414 & -5.173452 \\ \mathrm{H} & -0.462882 & -4.264327 & -6.080031 \\ \mathrm{H} & 0.664126 & -5.44776 & -5.417788 \\ \mathrm{H} & -1.033568 & -5.497606 & -4.924018\end{array}$

\begin{tabular}{llll} 
TI(TFA) ${ }_{3}$ - 0 -xylene C3 C-H activation transition state \\
Electronic Energy & \multicolumn{3}{c}{-2061.983135} \\
Electronic and Zero-point Energy & -2061.74755999 \\
Enthalpy & & -2061.71514799 \\
Free Energy & & -2061.81682699 \\
O & 1.736435 & -0.872613 & -1.150145 \\
C & 2.69922 & -0.110346 & -1.333141 \\
O & 2.805261 & 1.112311 & -1.030107 \\
C & 3.973156 & -0.689475 & -1.988146 \\
H & 1.741069 & 1.573256 & -0.45752 \\
O & -2.65065 & 0.268794 & 0.470493 \\
C & -2.870308 & 0.382185 & -0.754471 \\
O & -2.001325 & 0.322602 & -1.657745 \\
C & -4.311789 & 0.651983 & -1.227429 \\
F & -4.666626 & -0.214412 & -2.178331 \\
F & -4.382709 & 1.884598 & -1.747112 \\
F & -5.196989 & 0.569686 & -0.239213 \\
F & 3.789647 & -1.922015 & -2.447394 \\
F & 4.373185 & 0.080892 & -3.003171 \\
F & 4.960481 & -0.720609 & -1.083749 \\
TI & -0.242066 & 0.000927 & -0.002318 \\
O & 0.354237 & -1.684897 & 1.673531 \\
C & -0.220186 & -2.548345 & 0.971502 \\
O & -0.813991 & -2.312128 & -0.106265 \\
C & -0.158023 & -3.999169 & 1.481799 \\
F & -0.855011 & -4.835171 & 0.72042
\end{tabular}




$\begin{array}{llll}\text { F } & -0.632994 & -4.071079 & 2.72798 \\ \text { F } & 1.11358 & -4.410545 & 1.50279 \\ \mathrm{C} & 0.707653 & 2.090756 & 0.220892 \\ \mathrm{C} & 1.14783 & 2.410081 & 1.537052 \\ \mathrm{C} & -0.038984 & 3.033853 & -0.534902 \\ \mathrm{C} & 0.910291 & 3.699477 & 2.043569 \\ \mathrm{C} & -0.270005 & 4.294738 & -0.025562 \\ \mathrm{H} & -0.391548 & 2.768982 & -1.531587 \\ \mathrm{C} & 0.201631 & 4.608512 & 1.253699 \\ \mathrm{H} & -0.812676 & 5.038176 & -0.604462 \\ \mathrm{H} & 0.008192 & 5.602574 & 1.65647 \\ \mathrm{C} & 1.904111 & 1.410409 & 2.347745 \\ \mathrm{H} & 1.305912 & 1.061823 & 3.201992 \\ \mathrm{H} & 2.186144 & 0.526426 & 1.76322 \\ \mathrm{H} & 2.821583 & 1.845287 & 2.763451 \\ \mathrm{C} & 1.393209 & 4.093137 & 3.404619 \\ \mathrm{H} & 2.488163 & 4.046983 & 3.472052 \\ \mathrm{H} & 1.086672 & 5.114724 & 3.651282 \\ \mathrm{H} & 0.999352 & 3.425979 & 4.18227\end{array}$

TI(TFA)3- $m$-xylene $\mathrm{C} 2 \mathrm{C}-\mathrm{H}$ activation transition state

\begin{tabular}{llll} 
Electronic Energy & \multicolumn{3}{l}{-2061.988046} \\
Electronic and Zero-point Energy & -2061.752481 \\
Enthalpy & & -2061.720080 \\
Free Energy & & -2061.821864 \\
O & 1.736435 & -0.872613 & -1.150145 \\
C & 2.69922 & -0.110346 & -1.333141 \\
O & 2.805261 & 1.112311 & -1.030107 \\
C & 3.973156 & -0.689475 & -1.988146 \\
H & 1.741069 & 1.573256 & -0.45752 \\
O & -2.65065 & 0.268794 & 0.470493 \\
C & -2.870308 & 0.382185 & -0.754471 \\
O & -2.001325 & 0.322602 & -1.657745 \\
C & -4.311789 & 0.651983 & -1.227429 \\
F & -4.666626 & -0.214412 & -2.178331 \\
F & -4.382709 & 1.884598 & -1.747112 \\
F & -5.196989 & 0.569686 & -0.239213 \\
F & 3.789647 & -1.922015 & -2.447394 \\
F & 4.373185 & 0.080892 & -3.003171 \\
F & 4.960481 & -0.720609 & -1.083749 \\
TI & -0.242066 & 0.000927 & -0.002318 \\
O & 0.354237 & -1.684897 & 1.673531
\end{tabular}




$\begin{array}{llll}\text { C } & -0.220186 & -2.548345 & 0.971502 \\ \text { O } & -0.813991 & -2.312128 & -0.106265 \\ \text { C } & -0.158023 & -3.999169 & 1.481799 \\ \text { F } & -0.855011 & -4.835171 & 0.72042 \\ \text { F } & -0.632994 & -4.071079 & 2.72798 \\ \text { F } & 1.11358 & -4.410545 & 1.50279 \\ \text { C } & 0.707653 & 2.090756 & 0.220892 \\ \text { C } & 1.14783 & 2.410081 & 1.537052 \\ \text { C } & -0.038984 & 3.033853 & -0.534902 \\ \text { C } & 0.910291 & 3.699477 & 2.043569 \\ \mathrm{C} & -0.270005 & 4.294738 & -0.025562 \\ \mathrm{H} & -0.391548 & 2.768982 & -1.531587 \\ \mathrm{C} & 0.201631 & 4.608512 & 1.253699 \\ \mathrm{H} & -0.812676 & 5.038176 & -0.604462 \\ \mathrm{H} & 0.008192 & 5.602574 & 1.65647 \\ \mathrm{C} & 1.904111 & 1.410409 & 2.347745 \\ \mathrm{H} & 1.305912 & 1.061823 & 3.201992 \\ \mathrm{H} & 2.186144 & 0.526426 & 1.76322 \\ \mathrm{H} & 2.821583 & 1.845287 & 2.763451 \\ \mathrm{C} & 1.393209 & 4.093137 & 3.404619 \\ \mathrm{H} & 2.488163 & 4.046983 & 3.472052 \\ \mathrm{H} & 1.086672 & 5.114724 & 3.651282 \\ \mathrm{H} & 0.999352 & 3.425979 & 4.18227\end{array}$

$\mathrm{TI}(\mathrm{TFA})_{3}-p$-xylene $\mathrm{C}-\mathrm{H}$ activation transition state

\begin{tabular}{llll} 
Electronic Energy & & \multicolumn{2}{l}{-2061.98339953} \\
Electronic and Zero-point Energy & -2061.74767653 \\
Enthalpy & & -2061.71523253 \\
Free Energy & & -2061.81656853 \\
O & -2.111138 & 0.266981 & -0.789534 \\
C & -2.779532 & -0.752426 & -1.025321 \\
O & -2.460167 & -1.962508 & -0.851195 \\
C & -4.202656 & -0.571506 & -1.599494 \\
H & -1.300997 & -2.094188 & -0.25929 \\
O & 2.51318 & 0.615694 & 0.55682 \\
C & 2.739323 & 0.543722 & -0.668642 \\
O & 1.89794 & 0.224165 & -1.545196 \\
C & 4.154524 & 0.865028 & -1.187547 \\
F & 4.105702 & 1.850652 & -2.087278 \\
F & 4.672096 & -0.212553 & -1.789547 \\
F & 4.982046 & 1.231854 & -0.214042 \\
F & -5.100058 & -1.12968 & -0.779091
\end{tabular}




$\begin{array}{llll}\text { F } & -4.521892 & 0.707737 & -1.758139 \\ \mathrm{~F} & -4.301593 & -1.17959 & -2.786703 \\ \mathrm{TI} & 0.153826 & -0.01108 & 0.1306 \\ \mathrm{O} & -0.549166 & 1.494088 & 1.938986 \\ \mathrm{C} & -0.398265 & 2.460571 & 1.161109 \\ \mathrm{O} & -0.026108 & 2.368118 & -0.034008 \\ \mathrm{C} & -0.704349 & 3.88176 & 1.666883 \\ \mathrm{~F} & 0.315987 & 4.699934 & 1.40299 \\ \mathrm{~F} & -0.935754 & 3.91131 & 2.975812 \\ \mathrm{~F} & -1.786916 & 4.355965 & 1.041411 \\ \mathrm{C} & -0.197268 & -2.260946 & 0.464064 \\ \mathrm{C} & -0.616587 & -2.59558 & 1.782029 \\ \mathrm{C} & 0.796793 & -3.042081 & -0.18827 \\ \mathrm{C} & -0.106661 & -3.764522 & 2.349028 \\ \mathrm{C} & 1.314127 & -4.185592 & 0.394436 \\ \mathrm{C} & 0.838651 & -4.527139 & 1.675094 \\ \mathrm{H} & 1.231291 & -5.424842 & 2.153819 \\ \mathrm{H} & -0.446557 & -4.072726 & 3.337094 \\ \mathrm{C} & -1.605664 & -1.759053 & 2.523437 \\ \mathrm{H} & -1.099236 & -1.135088 & 3.27314 \\ \mathrm{H} & -2.159829 & -1.082675 & 1.858561 \\ \mathrm{H} & -2.327235 & -2.383662 & 3.0616 \\ \mathrm{C} & 2.333205 & -5.040983 & -0.290285 \\ \mathrm{H} & 3.235218 & -5.149597 & 0.324925 \\ \mathrm{H} & 1.945398 & -6.05117 & -0.471849 \\ \mathrm{H} & 2.630634 & -4.61538 & -1.254354 \\ \mathrm{H} & 1.127044 & -2.755191 & -1.188259\end{array}$

\begin{tabular}{llll} 
TI(TFA) ${ }_{3}$-mesitylene C-H activation transition state \\
Electronic Energy & \multicolumn{3}{c}{-2101.294244} \\
Electronic and Zero-point Energy & -2101.031304 & \\
Enthalpy & & -2100.997239 & \\
Free Energy & -2101.101453 \\
O & -0.945997 & 1.556646 & -1.348249 \\
$\mathrm{C}$ & -2.185664 & 1.586764 & -1.415134 \\
$\mathrm{O}$ & -3.024937 & 0.837837 & -0.840209 \\
$\mathrm{C}$ & -2.772358 & 2.685624 & -2.330603 \\
$\mathrm{H}$ & -2.454918 & -0.094651 & -0.12738 \\
$\mathrm{O}$ & 2.10334 & -1.551483 & -0.107688 \\
$\mathrm{C}$ & 2.10744 & -1.366544 & -1.349129 \\
$\mathrm{O}$ & 1.278298 & -0.674959 & -1.977722 \\
$\mathrm{C}$ & 3.23697 & -2.065734 & -2.128102 \\
& & & $\mathrm{~S} 43$
\end{tabular}




$\begin{array}{llll}\text { F } & 3.159658 & -1.854152 & -3.43819 \\ \mathrm{~F} & 3.185493 & -3.386495 & -1.917771 \\ \mathrm{~F} & 4.426787 & -1.629869 & -1.703889 \\ \mathrm{~F} & -2.335968 & 3.887015 & -1.939348 \\ \mathrm{~F} & -2.364708 & 2.491697 & -3.589206 \\ \mathrm{~F} & -4.102418 & 2.704896 & -2.324471 \\ \mathrm{TI} & 0.135842 & -0.102923 & 0.11747 \\ \mathrm{O} & 1.29732 & 0.770524 & 2.154338 \\ \mathrm{C} & 1.913602 & 1.555192 & 1.403239 \\ \mathrm{O} & 1.748097 & 1.642918 & 0.162493 \\ \mathrm{C} & 2.931806 & 2.493295 & 2.076254 \\ \mathrm{~F} & 3.775274 & 1.794886 & 2.839637 \\ \mathrm{~F} & 2.282879 & 3.356936 & 2.867267 \\ \mathrm{~F} & 3.644326 & 3.18964 & 1.197179 \\ \mathrm{C} & -1.898033 & -1.070623 & 0.581573 \\ \mathrm{C} & -2.346385 & -0.933475 & 1.931417 \\ \mathrm{C} & -2.125136 & -2.290672 & -0.132735 \\ \mathrm{C} & -3.095084 & -1.959093 & 2.490651 \\ \mathrm{C} & -2.875867 & -3.283457 & 0.469845 \\ \mathrm{C} & -3.356503 & -3.138494 & 1.781854 \\ \mathrm{H} & -3.48467 & -1.845771 & 3.502428 \\ \mathrm{C} & -2.069826 & 0.312383 & 2.704111 \\ \mathrm{H} & -1.125735 & 0.221922 & 3.259222 \\ \mathrm{H} & -1.977231 & 1.185507 & 2.042239 \\ \mathrm{H} & -2.859341 & 0.514126 & 3.435214 \\ \mathrm{H} & -3.099727 & -4.198086 & -0.080244 \\ \mathrm{C} & -1.607848 & -2.454102 & -1.523392 \\ \mathrm{H} & -1.605395 & -1.49816 & -2.06647 \\ \mathrm{H} & -0.572455 & -2.825678 & -1.512711 \\ \mathrm{H} & -2.203658 & -3.17468 & -2.092332 \\ \mathrm{C} & -4.152081 & -4.23863 & 2.396724 \\ \mathrm{H} & -5.104301 & -4.362849 & 1.863844 \\ \mathrm{H} & -3.62117 & -5.1954 & 2.315625 \\ \mathrm{H} & -372514 & -4.047767 & 3.451234\end{array}$

$\mathrm{TI}(\mathrm{TFA})_{3}$-1,2,3-trimethylbenzene $\mathrm{C}$ - $\mathrm{H}$ activation transition state

Electronic Energy

Electronic and Zero-point Energy

Enthalpy

Free Energy

$\mathrm{O}$

C
$-2101.28630201$

$-2101.02193711$

$-2100.98837111$

$-2101.09180211$
0.125961
0.224085
$-0.630699 \quad-0.026616$ 


\begin{tabular}{|c|c|c|c|}
\hline O & 3.206331 & -1.697071 & -0.704223 \\
\hline C & 4.562145 & -0.19799 & 0.567434 \\
\hline $\mathrm{H}$ & 2.068427 & -1.945821 & -1.220992 \\
\hline O & -2.455986 & -0.530809 & -0.789495 \\
\hline C & -2.463385 & -1.170821 & 0.28155 \\
\hline O & -1.446512 & -1.502501 & 0.941307 \\
\hline C & -3.811269 & -1.6357 & 0.866059 \\
\hline$F$ & -3.864217 & -2.974362 & 0.853604 \\
\hline$F$ & -4.852288 & -1.176258 & 0.178569 \\
\hline $\mathrm{F}$ & -3.937542 & -1.233253 & 2.132858 \\
\hline$F$ & 4.888271 & 1.017061 & 0.115022 \\
\hline$F$ & 4.474091 & -0.12854 & 1.899212 \\
\hline$F$ & 5.548299 & -1.034326 & 0.25843 \\
\hline $\mathrm{Tl}$ & 0.035513 & -0.442812 & -0.660172 \\
\hline O & 0.043655 & 1.819848 & -1.630325 \\
\hline C & -0.273286 & 2.265312 & -0.507704 \\
\hline O & -0.451417 & 1.56359 & 0.51927 \\
\hline C & -0.468224 & 3.781435 & -0.32389 \\
\hline$F$ & 0.387939 & 4.244176 & 0.591257 \\
\hline$F$ & -1.707346 & 4.034443 & 0.106645 \\
\hline$F$ & -0.275045 & 4.456276 & -1.452893 \\
\hline C & 0.919565 & -2.143564 & -1.918861 \\
\hline C & 1.194925 & -1.801506 & -3.276584 \\
\hline C & 0.265116 & -3.365799 & -1.62255 \\
\hline C & 0.893069 & -2.712731 & -4.297246 \\
\hline C & -0.005977 & -4.262481 & -2.631494 \\
\hline C & 0.29911 & -3.950381 & -3.968058 \\
\hline C & 1.878117 & -0.508 & -3.590372 \\
\hline $\mathrm{H}$ & 1.200311 & 0.190638 & -4.100976 \\
\hline $\mathrm{H}$ & 2.245232 & -0.005843 & -2.686584 \\
\hline $\mathrm{H}$ & 2.737667 & -0.660924 & -4.254026 \\
\hline $\mathrm{H}$ & -0.4671 & -5.221797 & -2.402387 \\
\hline C & -0.015652 & -4.94892 & -5.032091 \\
\hline $\mathrm{H}$ & 0.87064 & -5.183306 & -5.635829 \\
\hline $\mathrm{H}$ & -0.39354 & -5.88154 & -4.602825 \\
\hline $\mathrm{H}$ & -0.775043 & -4.564761 & -5.726664 \\
\hline C & 1.215364 & -2.386187 & -5.724902 \\
\hline $\mathrm{H}$ & 1.165487 & -1.310694 & -5.91989 \\
\hline $\mathrm{H}$ & 2.230094 & -2.716815 & -5.990651 \\
\hline $\mathrm{H}$ & 0.526335 & -2.87515 & -6.419861 \\
\hline $\mathrm{H}$ & 0.015245 & -3.61256 & -0.590688 \\
\hline
\end{tabular}




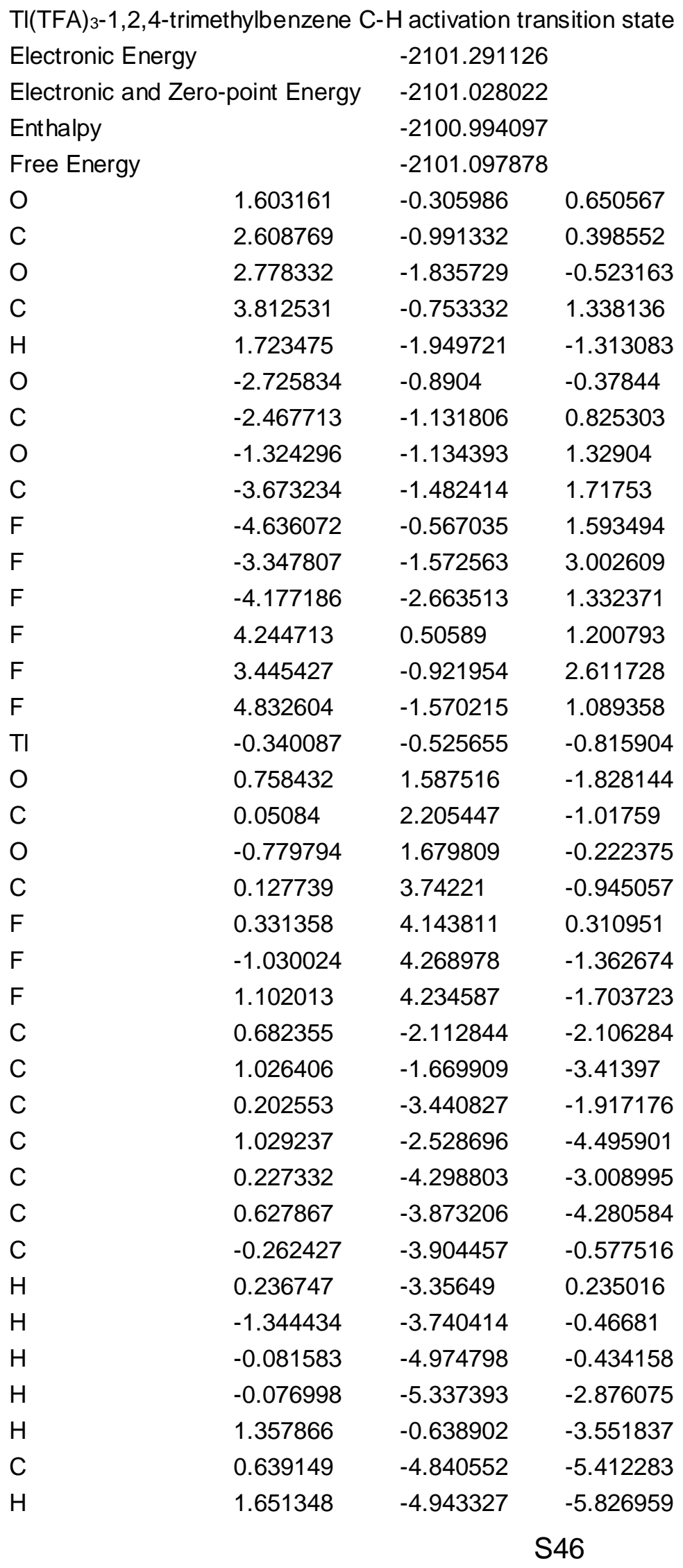




$\begin{array}{llll}\mathrm{H} & 0.293121 & -5.830679 & -5.101504 \\ \mathrm{H} & 0.002825 & -4.492305 & -6.236681 \\ \mathrm{C} & 1.455471 & -2.063615 & -5.852391 \\ \mathrm{H} & 2.3166 & -2.634987 & -6.222955 \\ \mathrm{H} & 0.652617 & -2.183822 & -6.591821 \\ \mathrm{H} & 1.73835 & -1.006257 & -5.831917\end{array}$

$\mathrm{TI}(\mathrm{TFA})_{3}$-durene $\mathrm{C}-\mathrm{H}$ activation transition state

Electronic Energy

Electronic and Zero-point Energy

Enthalpy

Free Energy

O

C

O

C

$\mathrm{H}$

O

C

O

C

$\mathrm{F}$

$\mathrm{F}$

F

$\mathrm{F}$

$\mathrm{F}$

$\mathrm{F}$

$\mathrm{TI}$

O

C

O

C

$\mathrm{F}$

$\mathrm{F}$

$\mathrm{F}$

C

C

C

C

C

C

C
$-2140.589785$

$-2140.299472$

$-2140.263688$

$-2140.373343$

$\begin{array}{ll}-0.098979 & 0.349539\end{array}$

$-0.776838 \quad-0.032849$

$-1.718866-0.879523$

$-0.469541 \quad 0.564076$

$-1.916776 \quad-1.403974$

$-0.409489-0.826052$

$-1.125781 \quad 0.194534$

$-1.595044 \quad 0.862788$

$-1.460714 \quad 0.629563$

$-0.344425 \quad 0.783906$

$-2.143334 \quad 1.769771$

$-2.199225-0.318409$

$-0.117014-0.408685$

$0.512754 \quad 1.456999$

$-1.558308 \quad 1.16237$

$-0.537097-0.623362$

$1.807904-1.343533$

$2.15504-0.17718$

$1.367103 \quad 0.775563$

$3.669098 \quad 0.076432$

$3.951264 \quad 1.319578$

$4.205928-0.760973$

$4.255872-0.148127$

$-2.156999-2.000452$

$-1.714885 \quad-3.355801$

$-3.463519 \quad-1.65087$

$-2.607647 \quad-4.366882$

$-4.348641-2.675538$

$-3.888544-3.995305$

$-0.347183-3.688458$ 


$\begin{array}{llll}\mathrm{H} & 0.663482 & 0.311441 & -4.032829 \\ \mathrm{H} & 1.953124 & 0.137365 & -2.826862 \\ \mathrm{H} & 2.213956 & -0.383558 & -4.497412 \\ \mathrm{C} & 0.650161 & -2.213484 & -5.810913 \\ \mathrm{H} & 0.104082 & -1.279084 & -5.992617 \\ \mathrm{H} & 1.680767 & -2.051228 & -6.154148 \\ \mathrm{H} & 0.20873 & -2.990195 & -6.444073 \\ \mathrm{C} & 0.460746 & -3.915838 & -0.226887 \\ \mathrm{H} & -0.555822 & -4.154167 & 0.114888 \\ \mathrm{H} & 1.05494 & -4.831425 & -0.106458 \\ \mathrm{H} & 0.87112 & -3.1635 & 0.458775 \\ \mathrm{H} & -0.115412 & -4.577804 & -4.787959 \\ \mathrm{C} & -0.30417 & -5.75242 & -2.379226 \\ \mathrm{H} & 0.508602 & -6.332969 & -1.922224 \\ \mathrm{H} & -1.146119 & -5.780922 & -1.675478 \\ \mathrm{H} & -0.611007 & -6.271301 & -3.293336\end{array}$

TI(TFA) $3-1,2,3,5$-tetramethylbenzene $\mathrm{C}$ - $\mathrm{H}$ activation transition state

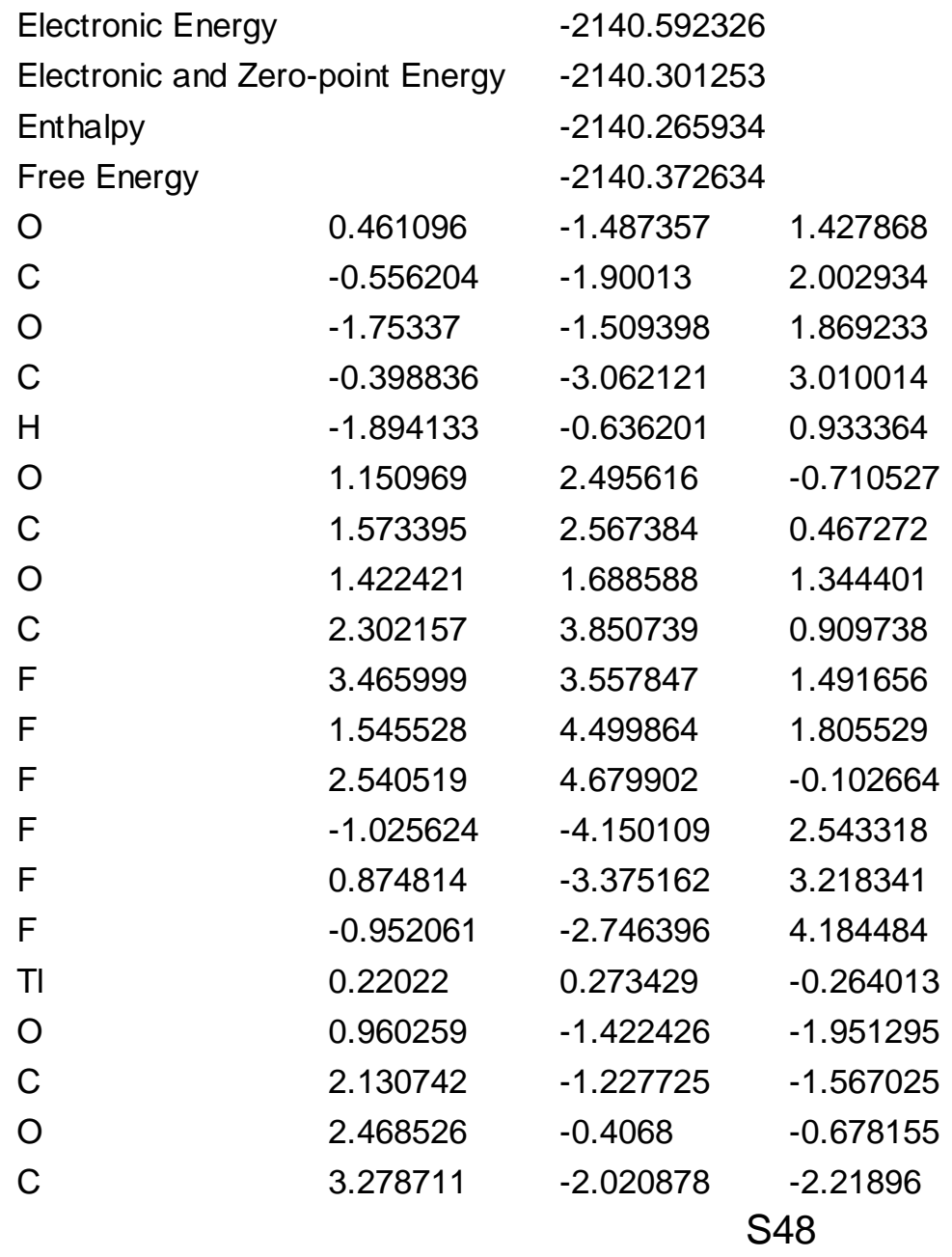




$\begin{array}{llll}\text { F } & 2.83748 & -2.922424 & -3.091707 \\ \text { F } & 3.995985 & -2.655713 & -1.289377 \\ \text { F } & 4.094122 & -1.181005 & -2.865964 \\ \mathrm{C} & -2.080842 & 0.219049 & -0.08809 \\ \mathrm{C} & -2.767745 & -0.516273 & -1.105875 \\ \mathrm{C} & -2.628209 & 1.433649 & 0.421351 \\ \mathrm{C} & -4.007876 & -0.071425 & -1.569099 \\ \mathrm{C} & -3.881292 & 1.817985 & -0.02235 \\ \mathrm{C} & -4.567528 & 1.099833 & -1.010509 \\ \mathrm{C} & -2.208377 & -1.820743 & -1.577629 \\ \mathrm{H} & -1.802212 & -1.746273 & -2.595939 \\ \mathrm{H} & -1.400499 & -2.17351 & -0.927208 \\ \mathrm{H} & -2.98091 & -2.598319 & -1.595286 \\ \mathrm{C} & -5.911895 & 1.57993 & -1.445876 \\ \mathrm{H} & -6.666913 & 0.788267 & -1.355143 \\ \mathrm{H} & -6.240401 & 2.438072 & -0.851992 \\ \mathrm{H} & -5.902623 & 1.882571 & -2.501577 \\ \mathrm{C} & -4.75821 & -0.842354 & -2.614597 \\ \mathrm{H} & -4.085489 & -1.395669 & -3.276394 \\ \mathrm{H} & -5.444651 & -1.573131 & -2.162731 \\ \mathrm{H} & -5.363023 & -0.183815 & -3.246332 \\ \mathrm{C} & -1.909472 & 2.231945 & 1.458368 \\ \mathrm{H} & -1.233056 & 2.964282 & 0.994278 \\ \mathrm{H} & -2.610485 & 2.796195 & 2.08213 \\ \mathrm{H} & -1.304831 & 1.589529 & 2.114552 \\ \mathrm{H} & -4.347878 & 2.706158 & 0.404124\end{array}$

TI(TFA) 3 -1,2,3,4-tetramethylbenzene $\mathrm{C}-\mathrm{H}$ activation transition state

\begin{tabular}{llll} 
Electronic Energy & \multicolumn{3}{c}{-2140.585951} \\
Electronic and Zero-point Energy & -2140.295174 \\
Enthalpy & & -2140.259517 \\
Free Energy & & -2140.369705 \\
O & -1.275066 & -1.882096 & -0.981699 \\
C & -0.54443 & -2.855299 & -1.229972 \\
O & 0.709599 & -2.954564 & -1.107796 \\
C & -1.278237 & -4.117655 & -1.737479 \\
H & 1.230496 & -1.912581 & -0.599577 \\
O & 0.04698 & 2.581712 & 0.420154 \\
C & 0.113139 & 2.823352 & -0.802638 \\
O & 0.026621 & 1.971328 & -1.72164 \\
C & 0.328123 & 4.277974 & -1.265043 \\
F & 0.37531 & 5.13554 & -0.250245
\end{tabular}




$\begin{array}{llll}\text { F } & -0.657822 & 4.654714 & -2.083938 \\ \mathrm{~F} & 1.480473 & 4.372799 & -1.940702 \\ \mathrm{~F} & -2.111478 & -4.560441 & -0.788855 \\ \mathrm{~F} & -2.007865 & -3.822644 & -2.816607 \\ \mathrm{~F} & -0.446761 & -5.10551 & -2.053302 \\ \mathrm{TI} & -0.235619 & 0.165708 & -0.103453 \\ \mathrm{O} & -1.768464 & -0.039665 & 1.82395 \\ \mathrm{C} & -2.672426 & 0.437887 & 1.107993 \\ \mathrm{O} & -2.529028 & 0.795311 & -0.088532 \\ \mathrm{C} & -4.079698 & 0.629019 & 1.703197 \\ \mathrm{~F} & -4.360474 & 1.933651 & 1.786748 \\ \mathrm{~F} & -4.187526 & 0.102462 & 2.919282 \\ \mathrm{~F} & -4.998647 & 0.06048 & 0.919628 \\ \mathrm{C} & 1.805804 & -0.864779 & 0.049278 \\ \mathrm{C} & 2.148522 & -1.310943 & 1.356819 \\ \mathrm{C} & 2.758859 & -0.160736 & -0.72753 \\ \mathrm{C} & 3.463643 & -1.136171 & 1.807473 \\ \mathrm{C} & 4.061134 & 0.004767 & -0.296557 \\ \mathrm{C} & 4.416121 & -0.503395 & 0.978201 \\ \mathrm{C} & 1.131149 & -2.031544 & 2.186586 \\ \mathrm{H} & 0.840219 & -1.446919 & 3.070126 \\ \mathrm{H} & 5.21478 & -2.240497 & 1.619459 \\ \mathrm{H} & 1.516989 & -2.991977 & 2.549708 \\ \mathrm{C} & 5.820583 & -0.352747 & 1.466436 \\ \mathrm{H} & 6.165784 & -1.250852 & 1.990108 \\ \mathrm{H} & 6.522311 & -0.146045 & 0.654952 \\ \mathrm{H} & 5.895981 & 0.479237 & 2.182299 \\ \mathrm{C} & 3.871339 & -1.615656 & 3.169989 \\ \mathrm{H} & 4.527793 & -0.894405 & 3.669072 \\ \mathrm{H} & 3.01026 & -1.774337 & 3.823755 \\ \mathrm{H} & 4.41996 & -2.566866 & 3.116489 \\ \mathrm{H} & 2.471935 & 0.224176 & -1.707805 \\ \mathrm{C} & 5.050373 & 0.722375 & -1.164285 \\ \mathrm{H} & & 1.575522 & -0.643399 \\ \mathrm{H} & & 0.067017 & -1.484721 \\ \mathrm{H} & 1.104061 & -2.066556\end{array}$

$\mathrm{TI}(\mathrm{TFA})_{3}$-pentamethylbenzene $\mathrm{C}-\mathrm{H}$ activation transition state

Electronic Energy

$-2179.887854$

Electronic and Zero-point Energy $\quad-2179.568734$

Enthalpy

$-2179.531910$

Free Energy

$-2179.64241$ 


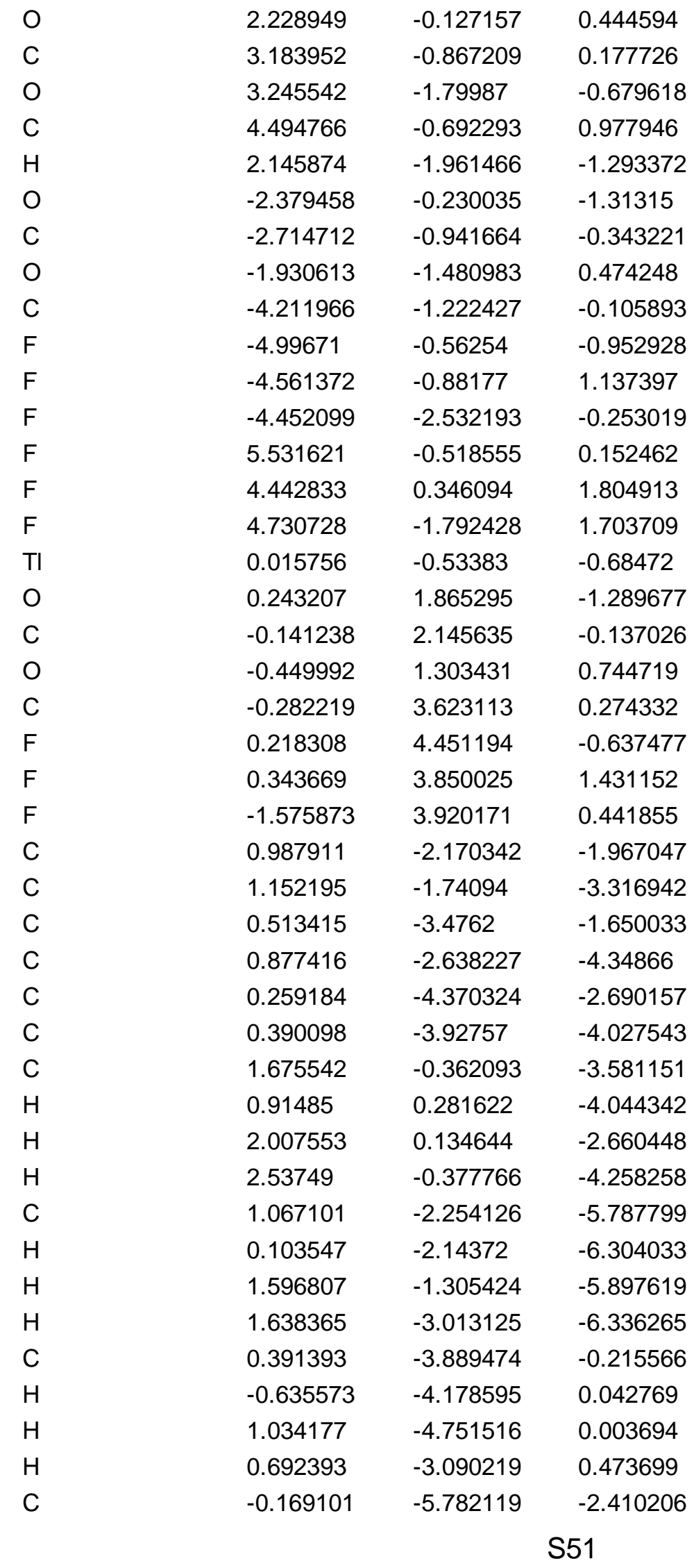




$\begin{array}{llll}\mathrm{H} & -0.009355 & -6.061572 & -1.366343 \\ \mathrm{H} & -1.237104 & -5.930031 & -2.622908 \\ \mathrm{H} & 0.3839 & -6.501347 & -3.025672 \\ \mathrm{C} & 0.030309 & -4.861243 & -5.138387 \\ \mathrm{H} & 0.910289 & -5.440842 & -5.45659 \\ \mathrm{H} & -0.736877 & -5.580611 & -4.840332 \\ \mathrm{H} & -0.335047 & -4.328001 & -6.020357\end{array}$

[TI(TFA) $)_{2}($ TFAH $\left.)\right]^{+}$-benzene $\mathrm{C}$ - $\mathrm{H}$ activation transition state Electronic Energy $\quad-1983.784834$

Electronic and Zero-point Energy $\quad-1983.590305$

Enthalpy $\quad-1983.561232$

Free Energy

$-1983.654471$

$\mathrm{O}$

$\begin{array}{lll}-2.279898 & -1.165243 & -0.290579\end{array}$

C

$\begin{array}{lll}-3.32627 & -0.523091 & -0.526355\end{array}$

O

$\begin{array}{lll}-3.488368 & 0.723053 & -0.578295\end{array}$

C

$-4.574082 \quad-1.39812 \quad-0.780112$

$\mathrm{H}$

$\begin{array}{lll}-2.401684 & 1.422104 & -0.285484\end{array}$

$\mathrm{F}$

$-5.664593 \quad-0.676022 \quad-1.006974$

$\mathrm{F}$

$\begin{array}{lll}-4.363381 & -2.187997 & -1.836757\end{array}$

$\mathrm{F}$

$-4.797896$

$-2.178599 \quad 0.280692$

$\mathrm{TI}$

$-0.32191$

0.075943

0.252265

O

0.912748

$-1.754609$

$-0.713097$

C

0.998716

$-2.277947$

0.428725

O

0.516631

$-1.765414$

1.463022

C

1.764709

$-3.61016$

0.519957

$\mathrm{F}$

1.775984

$-4.096686$

1.751749

F

3.025629

$-3.411937$

0.127298

$\mathrm{F}$

1.208882

$-4.506116$

$-0.292581$

C

$-1.318139$

2.110326

0.040403

C

$-0.779899$

2.826331

$-1.066659$

C

$-1.492131$

2.783225

1.28255

C

$-0.53682$

4.182494

$-0.961274$

$\mathrm{H}$

$-0.610811$

2.315112

$-2.013083$

C

$-1.246021$

4.140831

1.373955

$\mathrm{H}$

$-1.872545$

2.239622

2.14515

C

$-0.765395$

4.831631

0.257076

$\mathrm{H}$

$-0.166204$

4.742314

$-1.816023$

$\mathrm{H}$

$-1.418639$

4.66814

2.308563

O

1.987992

1.024605

0.169338

C

3.054514

0.69586

$-0.318616$

C

4.319089

1.552667

$-0.096567$ 


$\begin{array}{llll}\mathrm{O} & 3.323371 & -0.329512 & -1.066301 \\ \mathrm{H} & 2.530974 & -0.893716 & -1.233929 \\ \mathrm{~F} & 5.299248 & 0.805441 & 0.404559 \\ \mathrm{~F} & 4.725265 & 2.058548 & -1.260893 \\ \mathrm{~F} & 4.068893 & 2.548977 & 0.738098 \\ \mathrm{H} & -0.55965 & 5.897028 & 0.338402\end{array}$

$\mathrm{TI}(\mathrm{TFA}) 4^{-}$-benzene $\mathrm{C}-\mathrm{H}$ activation transition state

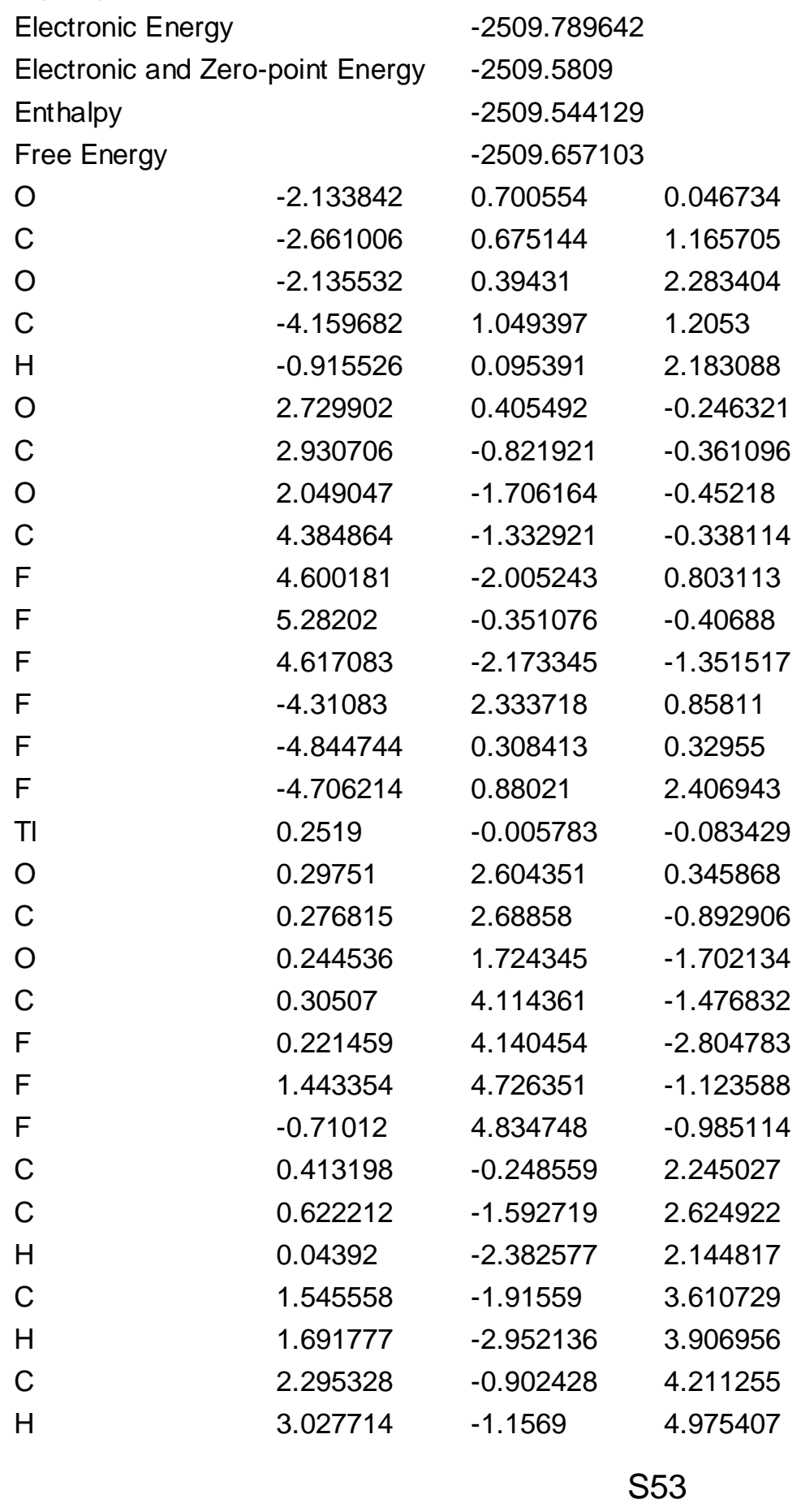




$\begin{array}{llll}\mathrm{C} & 2.1232 & 0.43205 & 3.838175 \\ \mathrm{H} & 2.715249 & 1.212215 & 4.311789 \\ \mathrm{C} & 1.20203 & 0.754592 & 2.850585 \\ \mathrm{H} & 1.073576 & 1.793812 & 2.546799 \\ \mathrm{C} & -1.194608 & -2.123445 & -1.26603 \\ \mathrm{C} & -1.943777 & -3.202051 & -2.07222 \\ \mathrm{O} & -1.210645 & -2.24653 & -0.031302 \\ \mathrm{~F} & -1.457741 & -4.415448 & -1.782243 \\ \mathrm{~F} & -3.242795 & -3.198269 & -1.745353 \\ \mathrm{~F} & -1.852536 & -3.028642 & -3.389061 \\ \mathrm{O} & -0.632531 & -1.202468 & -1.916076\end{array}$

Thallated benzene

\begin{tabular}{llll} 
Electronic Energy & \multicolumn{3}{l}{-1456.57681} \\
Electronic and Zero-point Energy & -1456.4310174 \\
Enthalpy & \multicolumn{3}{c}{-1456.4089354} \\
Free Energy & \multicolumn{3}{c}{-1456.4871414} \\
O & 3.032846 & 0.746168 & 0.465441 \\
C & 3.372417 & 0.183944 & -0.610048 \\
O & 2.684016 & -0.628948 & -1.251945 \\
C & 4.783846 & 0.540972 & -1.115969 \\
F & 4.942081 & 1.865206 & -1.18761 \\
F & 5.039497 & 0.027666 & -2.316138 \\
F & 5.699322 & 0.065579 & -0.26047 \\
TI & 0.892113 & -0.337701 & 0.571314 \\
O & 0.160561 & 1.863916 & 1.387862 \\
C & -0.009865 & 2.241242 & 0.205806 \\
O & 0.182503 & 1.534361 & -0.809354 \\
C & -0.504236 & 3.68788 & 0.022513 \\
F & 0.32417 & 4.539113 & 0.632688 \\
F & -1.714696 & 3.823773 & 0.577611 \\
F & -0.594772 & 4.038862 & -1.25633 \\
C & 0.309214 & -2.422025 & 1.08815 \\
C & -0.575612 & -2.673149 & 2.131201 \\
C & 0.882471 & -3.4377 & 0.330734 \\
C & -0.896162 & -4.000861 & 2.419221 \\
H & -1.01405 & -1.868032 & 2.716084 \\
C & 0.547491 & -4.758806 & 0.633739 \\
H & 1.574478 & -3.220287 & -0.481875 \\
C & -0.337083 & -5.036665 & 1.673414 \\
H & -1.586111 & -4.220068 & 3.231865 \\
H & 0.984226 & -5.568423 & 0.052395
\end{tabular}

S54 


\begin{tabular}{|c|c|c|c|}
\hline \multirow{2}{*}{\multicolumn{2}{|c|}{$\begin{array}{l}\mathrm{H} \\
\text { ortho thallated methylbenzoate }\end{array}$}} & -6.068544 & \multirow[t]{2}{*}{1.905067} \\
\hline & & & \\
\hline \multicolumn{2}{|c|}{ Electronic Energy } & \multicolumn{2}{|c|}{-1684.435311} \\
\hline \multicolumn{2}{|c|}{ Electronic and Zero-point Energy } & \multicolumn{2}{|c|}{-1684.246329} \\
\hline \multicolumn{2}{|c|}{ Enthalpy } & \multicolumn{2}{|c|}{-1684.220109} \\
\hline \multicolumn{2}{|c|}{ Free Energy } & \multicolumn{2}{|c|}{-1684.306659} \\
\hline TI & 0.65456 & 0.16593 & 0.917429 \\
\hline C & -0.773486 & 0.082287 & 2.634148 \\
\hline C & -1.22673 & -1.194484 & 2.974489 \\
\hline C & -1.190474 & 1.207749 & 3.327396 \\
\hline C & -2.117953 & -1.337257 & 4.045121 \\
\hline $\mathrm{H}$ & -2.479488 & -2.324698 & 4.321043 \\
\hline C & -2.080602 & 1.051715 & 4.393445 \\
\hline $\mathrm{H}$ & -0.837921 & 2.200629 & 3.056696 \\
\hline C & -2.538989 & -0.214428 & 4.748823 \\
\hline $\mathrm{H}$ & -3.231102 & -0.328709 & 5.57964 \\
\hline $\mathrm{H}$ & -2.414392 & 1.927777 & 4.945921 \\
\hline C & -0.767504 & -2.365152 & 2.188113 \\
\hline $\mathrm{O}$ & 0.000214 & -2.279727 & 1.230491 \\
\hline $\mathrm{O}$ & -1.262555 & -3.515226 & 2.600531 \\
\hline $\mathrm{O}$ & 2.895596 & -0.888607 & 0.740172 \\
\hline $\mathrm{O}$ & 1.921761 & 2.083919 & 0.111543 \\
\hline C & 2.657036 & -1.119668 & -0.462016 \\
\hline $\mathrm{O}$ & 1.58623 & -0.832528 & -1.054025 \\
\hline C & 1.001038 & 2.44964 & -0.662616 \\
\hline O & -0.100611 & 1.883849 & -0.798155 \\
\hline C & 3.732583 & -1.804788 & -1.324771 \\
\hline C & 1.299732 & 3.713694 & -1.491589 \\
\hline $\mathrm{F}$ & 4.84933 & -2.047748 & -0.644573 \\
\hline $\mathrm{F}$ & 3.272064 & -2.968666 & -1.796775 \\
\hline $\mathrm{F}$ & 4.04279 & -1.033759 & -2.371488 \\
\hline $\mathrm{F}$ & 2.396343 & 3.532967 & -2.234581 \\
\hline $\mathrm{F}$ & 1.526047 & 4.753167 & -0.677886 \\
\hline $\mathrm{F}$ & 0.297752 & 4.038785 & -2.303681 \\
\hline C & -0.897182 & -4.687845 & 1.85282 \\
\hline $\mathrm{H}$ & -1.41344 & -5.514988 & 2.34063 \\
\hline $\mathrm{H}$ & -1.227103 & -4.589549 & 0.814698 \\
\hline $\mathrm{H}$ & 0.185438 & -4.838388 & 1.889127 \\
\hline
\end{tabular}

meta thallated methylbenzoate

Electronic Energy

$-1684.425033$

S55 


\begin{tabular}{|c|c|c|c|}
\hline \multicolumn{2}{|c|}{ Electronic and Zero-point Energy } & \multicolumn{2}{|c|}{-1684.23633033} \\
\hline \multicolumn{2}{|c|}{ Enthalpy } & \multicolumn{2}{|c|}{-1684.20986033} \\
\hline \multicolumn{2}{|c|}{ Free Energy } & \multicolumn{2}{|c|}{-1684.29791033} \\
\hline $\mathrm{TI}$ & 0.116833 & 0.782228 & 0.352807 \\
\hline C & 1.810005 & -0.379107 & 1.209107 \\
\hline C & 2.965083 & -0.649042 & 0.491243 \\
\hline C & 1.614877 & -0.83465 & 2.509812 \\
\hline C & 3.964403 & -1.410968 & 1.11022 \\
\hline C & 2.624167 & -1.584233 & 3.115044 \\
\hline $\mathrm{H}$ & 0.697653 & -0.617038 & 3.055857 \\
\hline C & 3.790692 & -1.871429 & 2.417675 \\
\hline $\mathrm{H}$ & 4.580989 & -2.458007 & 2.881106 \\
\hline $\mathrm{H}$ & 2.491739 & -1.943807 & 4.132876 \\
\hline $\mathrm{O}$ & -0.345569 & 3.048355 & -0.516299 \\
\hline $\mathrm{O}$ & -1.572612 & 1.451011 & 1.985018 \\
\hline C & -1.256512 & 2.559806 & -1.218666 \\
\hline $\mathrm{O}$ & -1.528236 & 1.336281 & -1.285515 \\
\hline C & -2.167304 & 0.345931 & 1.937804 \\
\hline O & -1.840588 & -0.628301 & 1.230998 \\
\hline C & -2.09613 & 3.5493 & -2.046446 \\
\hline C & -3.418835 & 0.154687 & 2.81671 \\
\hline$F$ & -3.067773 & 2.948731 & -2.72541 \\
\hline$F$ & -2.648088 & 4.46092 & -1.242666 \\
\hline$F$ & -1.311218 & 4.190389 & -2.919129 \\
\hline$F$ & -3.270709 & -0.921237 & 3.599233 \\
\hline$F$ & -4.492534 & -0.049212 & 2.044685 \\
\hline$F$ & -3.668197 & 1.201184 & 3.599924 \\
\hline $\mathrm{H}$ & 3.106426 & -0.291113 & -0.524789 \\
\hline C & 5.224822 & -1.759515 & 0.410176 \\
\hline $\mathrm{O}$ & 6.108577 & -2.438378 & 0.903573 \\
\hline $\mathrm{O}$ & 5.295374 & -1.253262 & -0.821739 \\
\hline C & 6.486052 & -1.556245 & -1.561149 \\
\hline $\mathrm{H}$ & 6.576688 & -2.636459 & -1.710589 \\
\hline $\mathrm{H}$ & 6.374741 & -1.051104 & -2.521358 \\
\hline $\mathrm{H}$ & 7.368066 & -1.181182 & -1.033441 \\
\hline
\end{tabular}

para thallated methylbenzoate

$\begin{array}{lll}\text { Electronic Energy } & -1684.426359 \\ \text { Electronic and Zero-point Energy } & -1684.237509 & \\ \text { Enthalpy } & -1684.211139 & \\ \text { Free Energy } & -1684.298589 & \\ \text { TI } & -1.071308 & -0.153849 \\ & & \text { S56 }\end{array}$




\begin{tabular}{|c|c|c|c|}
\hline C & -2.761086 & -0.527692 & -0.965633 \\
\hline C & -2.852192 & 0.654249 & -1.692991 \\
\hline C & -3.861473 & -1.344704 & -0.72918 \\
\hline C & -4.092817 & 1.028379 & -2.204408 \\
\hline C & -5.09625 & -0.956677 & -1.241411 \\
\hline $\mathrm{H}$ & -3.778797 & -2.26726 & -0.160298 \\
\hline C & -5.213558 & 0.225505 & -1.976045 \\
\hline $\mathrm{H}$ & -5.976887 & -1.572918 & -1.072546 \\
\hline 0 & 0.965018 & 0.557274 & -1.107386 \\
\hline 0 & -0.159744 & -2.756931 & 1.535889 \\
\hline C & 1.703851 & -0.430571 & -1.283345 \\
\hline 0 & 1.401726 & -1.623746 & -1.023178 \\
\hline C & 0.445018 & -1.880101 & 2.19047 \\
\hline 0 & 0.520384 & -0.669406 & 1.872885 \\
\hline C & 3.093642 & -0.146237 & -1.885195 \\
\hline C & 1.191794 & -2.290887 & 3.473029 \\
\hline$F$ & 2.954583 & 0.359666 & -3.117343 \\
\hline$F$ & 3.742995 & 0.756878 & -1.145498 \\
\hline$F$ & 3.852791 & -1.234731 & -1.971392 \\
\hline $\mathrm{F}$ & 0.935444 & -3.546062 & 3.829303 \\
\hline $\mathrm{F}$ & 0.854547 & -1.492009 & 4.489116 \\
\hline $\mathrm{F}$ & 2.509843 & -2.172922 & 3.278964 \\
\hline $\mathrm{H}$ & -1.982299 & 1.284136 & -1.869366 \\
\hline $\mathrm{H}$ & -4.184189 & 1.950693 & -2.772438 \\
\hline C & -6.557101 & 0.59402 & -2.48861 \\
\hline $\mathrm{O}$ & -7.579472 & -0.011079 & -2.215731 \\
\hline $\mathrm{O}$ & -6.534058 & 1.663244 & -3.284162 \\
\hline C & -7.800275 & 2.103785 & -3.790404 \\
\hline $\mathrm{H}$ & -7.583912 & 2.974455 & -4.410726 \\
\hline $\mathrm{H}$ & -8.266197 & 1.316081 & -4.390373 \\
\hline $\mathrm{H}$ & -8.464323 & 2.379173 & -2.9651 \\
\hline
\end{tabular}

ortho thallated toluene

$\begin{array}{llll}\text { Electronic Energy } & & -1495.879899 & \\ \text { Electronic and Zero-point Energy } & -1495.707087 & \\ \text { Enthalpy } & & -1495.683237 & \\ \text { Free Energy } & -1495.765519 & \\ \text { O } & 2.060725 & 1.554398 & -0.761187 \\ \text { C } & 1.695319 & 2.068615 & 0.327994 \\ \text { O } & 0.634136 & 1.813044 & 0.927269 \\ \text { C } & 2.682713 & 3.073002 & 0.953364 \\ \text { F } & 3.81758 & 2.446501 & 1.283232\end{array}$




$\begin{array}{llll}\text { F } & 2.194106 & 3.646838 & 2.049338 \\ \text { F } & 2.987552 & 4.038912 & 0.080324 \\ \text { TI } & 0.137812 & 0.104607 & -0.880135 \\ \text { O } & 1.902261 & -1.505169 & -1.660322 \\ \text { C } & 2.121736 & -1.793572 & -0.464664 \\ \text { O } & 1.51279 & -1.312668 & 0.520979 \\ \text { C } & 3.229639 & -2.832277 & -0.205554 \\ \text { F } & 4.376859 & -2.435707 & -0.763346 \\ \text { F } & 2.889239 & -4.00258 & -0.760505 \\ \text { F } & 3.447274 & -3.038494 & 1.089972 \\ \mathrm{C} & -1.988201 & 0.14382 & -1.570766 \\ \mathrm{C} & -2.468595 & -0.917021 & -2.341098 \\ \mathrm{C} & -2.760007 & 1.232366 & -1.1847 \\ \mathrm{C} & -3.812047 & -0.837111 & -2.730376 \\ \mathrm{C} & -4.094671 & 1.277866 & -1.591276 \\ \mathrm{H} & -2.345781 & 2.03482 & -0.576628 \\ \mathrm{C} & -4.612473 & 0.242608 & -2.36372 \\ \mathrm{H} & -4.230419 & -1.643583 & -3.332088 \\ \mathrm{H} & -4.718612 & 2.120503 & -1.300763 \\ \mathrm{H} & -5.6522 & 0.271643 & -2.683334 \\ \mathrm{C} & -1.612984 & -2.07976 & -2.738184 \\ \mathrm{H} & -0.805025 & -1.771366 & -3.415663 \\ \mathrm{H} & -1.144956 & -2.549051 & -1.861148 \\ \mathrm{H} & -2.198924 & -2.849275 & -3.250374\end{array}$

meta thallated toluene

\begin{tabular}{llll} 
Electronic Energy & \multicolumn{3}{l}{-1495.877764} \\
Electronic and Zero-point Energy & -1495.704548 & \\
Enthalpy & & -1495.680925 & \\
Free Energy & & -1495.761276 & \\
O & -3.171641 & 0.437835 & 0.053724 \\
C & -3.290457 & -0.52147 & 0.861944 \\
O & -2.418902 & -1.370979 & 1.115825 \\
C & -4.666769 & -0.632499 & 1.545395 \\
F & -5.018084 & 0.528724 & 2.103814 \\
F & -4.692051 & -1.570931 & 2.48804 \\
F & -5.595609 & -0.941692 & 0.629391 \\
TI & -0.907047 & -0.112815 & -0.54832 \\
O & -0.653766 & 2.326451 & -0.429638 \\
C & -0.482021 & 2.255367 & 0.810263 \\
O & -0.453428 & 1.195166 & 1.472093 \\
C & -0.286288 & 3.594608 & 1.544087 \\
& & & S58
\end{tabular}




$\begin{array}{llll}\text { F } & -1.309104 & 4.415633 & 1.293071 \\ \text { F } & 0.832012 & 4.188045 & 1.108316 \\ \text { F } & -0.187162 & 3.442762 & 2.860819 \\ \text { C } & -0.03923 & -1.752 & -1.782556 \\ \text { C } & 0.80723 & -1.467846 & -2.848545 \\ \text { C } & -0.395661 & -3.045391 & -1.425174 \\ \text { C } & 1.333872 & -2.524323 & -3.601172 \\ \text { C } & 0.133249 & -4.094687 & -2.181674 \\ \text { H } & -1.059889 & -3.250193 & -0.587121 \\ \text { C } & 0.983242 & -3.833707 & -3.250118 \\ \text { H } & -0.125159 & -5.120828 & -1.926766 \\ H & 1.389019 & -4.662581 & -3.830254 \\ \text { C } & 2.250645 & -2.265518 & -4.757394 \\ H & 3.206002 & -2.789771 & -4.629502 \\ H & 1.812428 & -2.625428 & -5.696995 \\ H & 2.464373 & -1.197716 & -4.87361 \\ \text { H } & 1.069331 & -0.443862 & -3.109837\end{array}$

para thallated toluene

\begin{tabular}{llll} 
Electronic Energy & \multicolumn{3}{l}{-1495.877898} \\
Electronic and Zero-point Energy & -1495.705072 \\
Enthalpy & & -1495.705072 \\
Free Energy & & -1495.76361 \\
O & -3.096342 & 0.654309 & 0.003711 \\
C & -3.293463 & -0.303868 & 0.796383 \\
O & -2.474619 & -1.199226 & 1.072263 \\
C & -4.69761 & -0.356175 & 1.428936 \\
F & -5.036824 & 0.827284 & 1.945905 \\
F & -4.787147 & -1.269191 & 2.39255 \\
F & -5.599621 & -0.662541 & 0.485141 \\
TI & -0.839788 & -0.022921 & -0.527036 \\
O & -0.475001 & 2.405982 & -0.423679 \\
C & -0.339801 & 2.332566 & 0.819386 \\
O & -0.374093 & 1.272958 & 1.484257 \\
C & -0.083236 & 3.657544 & 1.560187 \\
F & -0.924637 & 4.604752 & 1.143379 \\
F & 1.164385 & 4.073469 & 1.301807 \\
F & -0.212335 & 3.535021 & 2.877567 \\
C & -0.094781 & -1.679874 & -1.806493 \\
C & 0.749235 & -1.437488 & -2.885779 \\
C & -0.528516 & -2.95867 & -1.481775 \\
C & 1.166706 & -2.521715 & -3.654465
\end{tabular}




$\begin{array}{llll}\mathrm{H} & 1.083976 & -0.433699 & -3.137172 \\ \mathrm{C} & -0.09659 & -4.027762 & -2.268432 \\ \mathrm{H} & -1.189004 & -3.140571 & -0.63508 \\ \mathrm{C} & 0.751766 & -3.826521 & -3.359755 \\ \mathrm{H} & 1.829139 & -2.349014 & -4.503098 \\ \mathrm{H} & -0.429068 & -5.03597 & -2.022697 \\ \mathrm{C} & 1.211422 & -4.973615 & -4.205856 \\ \mathrm{H} & 0.867075 & -4.864065 & -5.242252 \\ \mathrm{H} & 2.306717 & -5.028686 & -4.23846 \\ \mathrm{H} & 0.836157 & -5.930273 & -3.827\end{array}$

\begin{tabular}{|c|c|c|c|}
\hline \multicolumn{4}{|c|}{ TI(TFA)2+-benzene $\pi$-complex } \\
\hline \multicolumn{2}{|c|}{ Electronic Energy } & \multicolumn{2}{|c|}{-1456.97435689} \\
\hline \multicolumn{2}{|c|}{ Electronic and Zero-point Energy } & \multicolumn{2}{|c|}{-1456.81533954} \\
\hline \multicolumn{2}{|c|}{ Enthalpy } & \multicolumn{2}{|c|}{-1456.79383254} \\
\hline \multicolumn{2}{|c|}{ Free Energy } & \multicolumn{2}{|c|}{-1456.86817654} \\
\hline O & -2.486352 & -0.816506 & -0.708068 \\
\hline C & -2.773858 & 0.162306 & 0.01947 \\
\hline O & -1.940975 & 0.784217 & 0.72341 \\
\hline C & -4.230444 & 0.663708 & 0.074139 \\
\hline $\mathrm{H}$ & 0.606501 & -3.309771 & 1.628014 \\
\hline $\mathrm{F}$ & -4.680347 & 0.58397 & 1.325919 \\
\hline $\mathrm{F}$ & -4.2776 & 1.937966 & -0.309549 \\
\hline $\mathrm{F}$ & -5.028157 & -0.046942 & -0.710723 \\
\hline $\mathrm{TI}$ & -0.196744 & -0.597155 & -0.062243 \\
\hline $\mathrm{O}$ & 1.42386 & 0.748605 & 1.076389 \\
\hline C & 1.624362 & 1.388778 & 0.020354 \\
\hline $\mathrm{O}$ & 1.045814 & 1.158872 & -1.070529 \\
\hline C & 2.669074 & 2.521894 & 0.012555 \\
\hline$F$ & 3.182093 & 2.736472 & 1.216357 \\
\hline$F$ & 2.117708 & 3.651029 & -0.42526 \\
\hline $\mathrm{F}$ & 3.663093 & 2.190604 & -0.816568 \\
\hline C & 1.308718 & -2.828207 & 0.951106 \\
\hline C & 2.520077 & -2.335586 & 1.409233 \\
\hline C & 0.980931 & -2.722571 & -0.435145 \\
\hline C & 3.421549 & -1.765118 & 0.506032 \\
\hline C & 1.920685 & -2.150489 & -1.340045 \\
\hline $\mathrm{H}$ & 0.121396 & -3.271715 & -0.826142 \\
\hline C & 3.126602 & -1.669471 & -0.85865 \\
\hline $\mathrm{H}$ & 1.679777 & -2.102845 & -2.399533 \\
\hline $\mathrm{H}$ & 3.846784 & -1.227477 & -1.541873 \\
\hline $\mathrm{H}$ & 2.772164 & -2.402754 & 2.463819 \\
\hline & & & \\
\hline
\end{tabular}




\begin{tabular}{|c|c|c|c|}
\hline $\mathrm{H}$ & 4.373565 & -1.386774 & 0.87188 \\
\hline \multicolumn{4}{|c|}{$\left[\mathrm{TI}(\mathrm{TFA})_{2}(\mathrm{TFAH})\right]^{+}$-benzene $\pi$-complex } \\
\hline \multicolumn{2}{|c|}{ Electronic Energy } & \multicolumn{2}{|c|}{-1983.816345} \\
\hline \multicolumn{2}{|c|}{ Electronic and Zero-point Energy } & \multicolumn{2}{|c|}{-1983.615904} \\
\hline \multicolumn{2}{|c|}{ Enthalpy } & \multicolumn{2}{|c|}{-1983.58675} \\
\hline \multicolumn{2}{|c|}{ Free Energy } & \multicolumn{2}{|c|}{-1983.679598} \\
\hline C & -1.226309 & 1.375851 & -0.008718 \\
\hline C & 0.011505 & 1.091373 & 0.633975 \\
\hline C & 0.702956 & 2.108358 & 1.271633 \\
\hline C & 0.197046 & 3.412401 & 1.254927 \\
\hline C & -1.011686 & 3.714811 & 0.623527 \\
\hline C & -1.725944 & 2.711846 & -0.015786 \\
\hline $\mathrm{H}$ & -1.677348 & 0.627408 & -0.666035 \\
\hline $\mathrm{H}$ & 0.400654 & 0.075479 & 0.616197 \\
\hline $\mathrm{H}$ & 1.645203 & 1.895652 & 1.770525 \\
\hline $\mathrm{H}$ & 0.753187 & 4.205831 & 1.749677 \\
\hline $\mathrm{H}$ & -1.386399 & 4.734461 & 0.624012 \\
\hline $\mathrm{H}$ & -2.652745 & 2.931394 & -0.541443 \\
\hline $\mathrm{TI}$ & -2.568456 & 0.734973 & 1.960234 \\
\hline O & -1.901794 & -1.58383 & 1.583674 \\
\hline C & -1.583381 & -2.460091 & 2.375421 \\
\hline O & -4.765322 & 0.078572 & 1.490913 \\
\hline C & -4.907222 & -0.419071 & 2.642024 \\
\hline O & -1.979011 & 2.335199 & 3.604601 \\
\hline C & -1.175804 & 1.558208 & 4.178542 \\
\hline O & -1.203802 & -2.339643 & 3.602853 \\
\hline O & -4.019574 & -0.411655 & 3.517212 \\
\hline O & -0.940217 & 0.389807 & 3.804049 \\
\hline C & -6.281247 & -1.046018 & 2.944819 \\
\hline C & -1.672091 & -3.929163 & 1.917314 \\
\hline C & -0.380807 & 2.140391 & 5.361331 \\
\hline $\mathrm{F}$ & 0.564872 & 2.947433 & 4.865542 \\
\hline$F$ & 0.209792 & 1.190627 & 6.074703 \\
\hline$F$ & -0.89224 & -4.725321 & 2.633688 \\
\hline$F$ & -2.937823 & -4.332584 & 2.050566 \\
\hline $\mathrm{F}$ & -1.331672 & -4.024139 & 0.638134 \\
\hline $\mathrm{F}$ & -6.581828 & -1.953169 & 2.016674 \\
\hline$F$ & -6.304897 & -1.630288 & 4.134304 \\
\hline$F$ & -7.215156 & -0.093862 & 2.919858 \\
\hline$F$ & -1.164246 & 2.853946 & 6.162101 \\
\hline $\mathrm{H}$ & -1.127353 & -1.393993 & 3.886523 \\
\hline
\end{tabular}




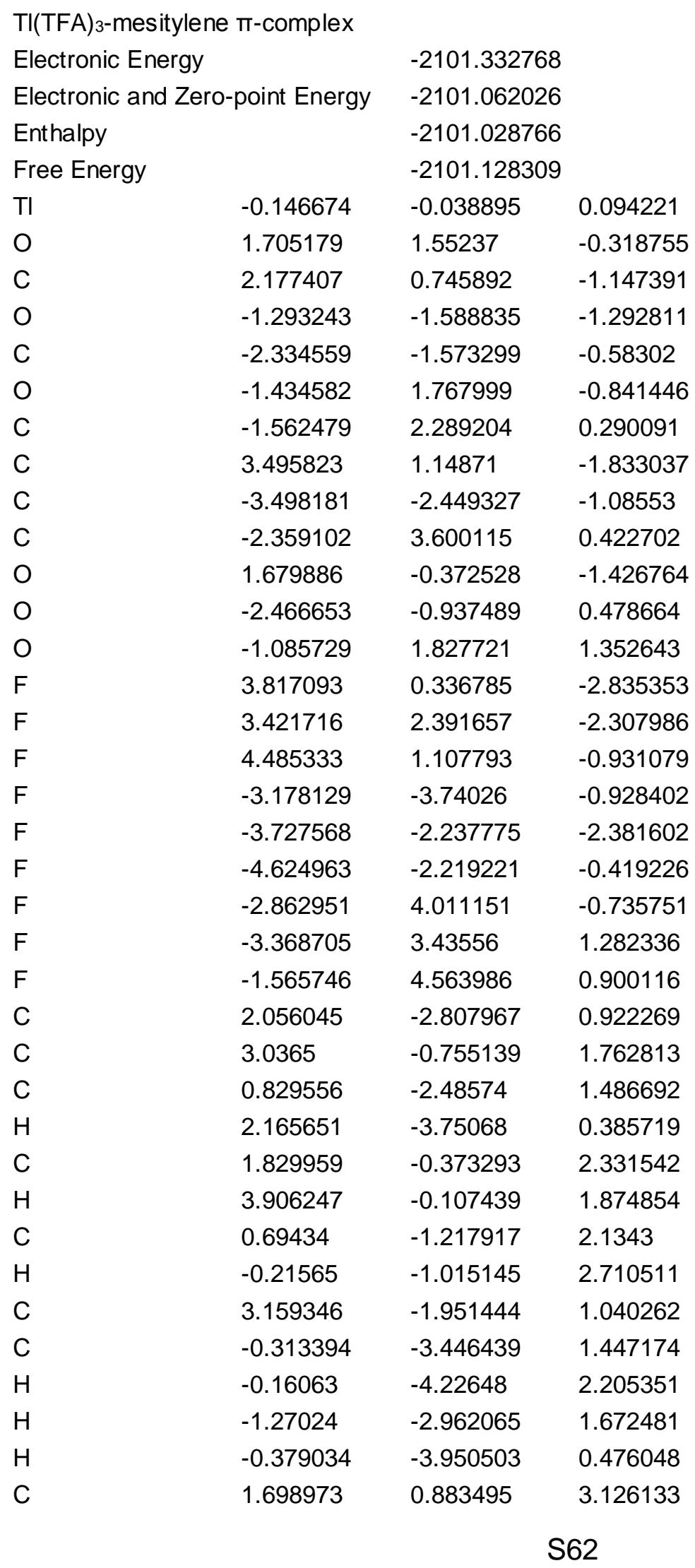




$\begin{array}{llll}\mathrm{H} & 0.769667 & 1.418517 & 2.894378 \\ \mathrm{H} & 1.671641 & 0.648703 & 4.199086 \\ \mathrm{H} & 2.542999 & 1.557258 & 2.947771 \\ \mathrm{C} & 4.443705 & -2.296668 & 0.366394 \\ \mathrm{H} & 4.37496 & -2.063432 & -0.706401 \\ \mathrm{H} & 5.286834 & -1.732102 & 0.777555 \\ \mathrm{H} & 4.660907 & -3.368231 & 0.442669\end{array}$

TI(TFA) 3 -1,2,3-trimethylbenzene $\pi$-complex

\begin{tabular}{llll} 
Electronic Energy & \multicolumn{3}{c}{-2101.325071} \\
Electronic and Zero-point Energy & -2101.055203 \\
Enthalpy & \multicolumn{3}{c}{-2101.021604} \\
Free Energy & \multicolumn{3}{c}{-2101.123012} \\
TI & 0.368667 & 0.450831 & 3.563755 \\
O & 2.672066 & 0.988203 & 4.22213 \\
C & 2.448983 & 2.19211 & 3.96493 \\
O & -1.633462 & 1.736643 & 3.697231 \\
C & -2.36528 & 0.716485 & 3.605241 \\
O & 0.175132 & -0.250327 & 5.852864 \\
C & 0.560222 & -1.402028 & 5.546344 \\
C & 3.580503 & 3.191794 & 4.274732 \\
C & -3.882966 & 0.976978 & 3.604396 \\
C & 0.65055 & -2.474833 & 6.646923 \\
O & 1.383134 & 2.632698 & 3.474137 \\
O & -1.948222 & -0.45151 & 3.482538 \\
O & 0.880379 & -1.766817 & 4.3905 \\
F & 3.180514 & 4.454472 & 4.164996 \\
F & 4.04093 & 3.003317 & 5.51114 \\
F & 4.59487 & 3.002068 & 3.422627 \\
F & -4.226504 & 1.561743 & 2.449076 \\
F & -4.223727 & 1.796975 & 4.598274 \\
F & -4.587018 & -0.143243 & 3.725244 \\
F & 0.302692 & -2.007639 & 7.840606 \\
F & -0.152994 & -3.499831 & 6.348651 \\
F & 1.900205 & -2.940529 & 6.725427 \\
C & 0.756607 & 1.90691 & 0.404585 \\
C & 2.767712 & 0.566128 & 0.833619 \\
C & -0.033435 & 0.81061 & 0.743153 \\
C & 1.96197 & -0.510086 & 1.196706 \\
C & 0.558305 & -0.403296 & 1.164926 \\
H & -0.062411 & -1.282964 & 1.348275 \\
C & 2.1633 & 1.78503 & 0.447288
\end{tabular}




$\begin{array}{llll}\mathrm{C} & 2.998308 & 2.986541 & 0.146044 \\ \mathrm{H} & 2.94104 & 3.707433 & 0.977149 \\ \mathrm{H} & 4.052359 & 2.745401 & -0.008611 \\ \mathrm{H} & 2.638032 & 3.515418 & -0.744026 \\ \mathrm{H} & -1.120056 & 0.888807 & 0.697923 \\ \mathrm{H} & 2.417684 & -1.444597 & 1.521696 \\ \mathrm{C} & 0.105466 & 3.21029 & 0.067969 \\ \mathrm{H} & 0.410473 & 3.995521 & 0.77358 \\ \mathrm{H} & 0.377654 & 3.560397 & -0.936179 \\ \mathrm{H} & -0.985261 & 3.125994 & 0.111784 \\ \mathrm{C} & 4.255595 & 0.412564 & 0.904543 \\ \mathrm{H} & 4.728123 & 0.563618 & -0.074462 \\ \mathrm{H} & 4.707087 & 1.135849 & 1.594402 \\ \mathrm{H} & 4.52271 & -0.589841 & 1.253567\end{array}$

TI(TFA)3-1,2,4-trimethylbenzene m-complex

\begin{tabular}{llll} 
Electronic Energy & \multicolumn{3}{c}{-2101.330964} \\
Electronic and Zero-point Energy & -2101.061147 \\
Enthalpy & \multicolumn{3}{c}{-2101.02755} \\
Free Energy & \multicolumn{3}{c}{-2101.129736} \\
TI & 0.104424 & 0.662202 & 3.417367 \\
O & 2.493214 & 0.969949 & 3.856442 \\
C & 2.29443 & 2.194454 & 4.015376 \\
O & -1.953814 & 1.899122 & 3.47085 \\
C & -2.629155 & 0.838984 & 3.446363 \\
O & 0.043436 & 0.076697 & 5.730554 \\
C & 0.428199 & -1.09087 & 5.478282 \\
C & 3.530518 & 3.049562 & 4.350706 \\
C & -4.158838 & 1.016439 & 3.506468 \\
C & 0.655649 & -2.061825 & 6.651286 \\
O & 1.18656 & 2.766644 & 3.881922 \\
O & -2.152274 & -0.312776 & 3.376318 \\
O & 0.667363 & -1.533643 & 4.332956 \\
F & 3.209642 & 4.278884 & 4.73808 \\
F & 4.253722 & 2.480106 & 5.314764 \\
F & 4.300949 & 3.143739 & 3.258462 \\
F & -4.573236 & 1.73812 & 2.459616 \\
F & -4.501823 & 1.669594 & 4.619076 \\
F & -4.803379 & -0.145205 & 3.490248 \\
F & 0.2985 & -1.540566 & 7.819958 \\
F & -0.041597 & -3.184965 & 6.463545 \\
F & 1.950791 & -2.387168 & 6.716808
\end{tabular}

S64 


$\begin{array}{llll}\text { C } & 0.874593 & 1.777674 & 0.654945 \\ \mathrm{C} & 2.939911 & 0.472441 & 0.807329 \\ \mathrm{C} & 0.146026 & 0.586195 & 0.900791 \\ \mathrm{C} & 2.216872 & -0.717096 & 1.052932 \\ \mathrm{C} & 0.828617 & -0.65109 & 1.059868 \\ \mathrm{C} & 2.260856 & 1.681113 & 0.605277 \\ \mathrm{C} & 0.1591 & 3.074806 & 0.472117 \\ \mathrm{H} & -0.259752 & 3.146832 & -0.540624 \\ \mathrm{H} & -0.679376 & 3.169872 & 1.173794 \\ \mathrm{H} & 0.831059 & 3.926254 & 0.620295 \\ \mathrm{H} & 0.241829 & -1.556761 & 1.224009 \\ \mathrm{C} & 4.430269 & 0.457386 & 0.827668 \\ \mathrm{H} & 4.848035 & 1.420093 & 0.518379 \\ \mathrm{H} & 4.793929 & 0.232935 & 1.841481 \\ \mathrm{H} & 4.837437 & -0.321442 & 0.170522 \\ \mathrm{H} & -0.942626 & 0.591769 & 0.795379 \\ \mathrm{C} & 2.918267 & -1.995742 & 1.376417 \\ \mathrm{H} & 3.632991 & -2.28607 & 0.596022 \\ \mathrm{H} & 3.486626 & -1.892803 & 2.312502 \\ \mathrm{H} & 2.204569 & -2.815035 & 1.508568 \\ \mathrm{H} & 2.842832 & 2.584428 & 0.418811\end{array}$

TI(TFA) 3 -durene $\pi$-complex

\begin{tabular}{llll} 
Electronic Energy & \multicolumn{3}{l}{-2140.633381} \\
Electronic and Zero-point Energy & -2140.33602 \\
Enthalpy & \multicolumn{3}{c}{-2140.300824} \\
Free Energy & \multicolumn{3}{c}{-2140.405849} \\
TI & 0.304084 & 0.353153 & 3.556297 \\
O & 2.620482 & 0.902539 & 4.204966 \\
C & 2.368345 & 2.106091 & 3.979775 \\
O & -1.73571 & 1.565473 & 3.748797 \\
C & -2.437928 & 0.5265 & 3.640709 \\
O & 0.130402 & -0.227352 & 5.883309 \\
C & 0.553727 & -1.379897 & 5.638445 \\
C & 3.518443 & 3.108954 & 4.187041 \\
C & -3.962932 & 0.740773 & 3.699597 \\
C & 0.736494 & -2.368552 & 6.804657 \\
O & 1.267604 & 2.544207 & 3.567102 \\
O & -1.988888 & -0.624407 & 3.476972 \\
O & 0.864044 & -1.804799 & 4.50078 \\
F & 3.102803 & 4.370778 & 4.148917 \\
F & 4.124578 & 2.90272 & 5.355945 \\
& & & S65
\end{tabular}




$\begin{array}{llll}\text { F } & 4.424324 & 2.939752 & 3.215524 \\ \text { F } & -4.344014 & 1.528678 & 2.687609 \\ \text { F } & -4.305713 & 1.341665 & 4.840738 \\ \text { F } & -4.636835 & -0.400982 & 3.611282 \\ \text { F } & 0.343517 & -1.86088 & 7.967448 \\ \text { F } & 0.037048 & -3.483235 & 6.576438 \\ \mathrm{~F} & 2.025703 & -2.705273 & 6.909014 \\ \mathrm{C} & 0.861747 & 1.98698 & 0.370678 \\ \mathrm{C} & 2.817204 & 0.575674 & 0.907252 \\ \mathrm{C} & -0.000484 & 0.920297 & 0.652506 \\ \mathrm{C} & 1.972554 & -0.493794 & 1.233628 \\ \mathrm{C} & 0.567063 & -0.296248 & 1.131107 \\ \mathrm{C} & 2.239349 & 1.78106 & 0.49704 \\ \mathrm{C} & -1.474657 & 1.033361 & 0.435116 \\ \mathrm{H} & -1.689143 & 1.193725 & -0.630427 \\ \mathrm{H} & -2.001035 & 0.123801 & 0.744645 \\ \mathrm{H} & -1.902867 & 1.891027 & 0.970886 \\ \mathrm{C} & 2.519663 & -1.809487 & 1.678715 \\ \mathrm{H} & 1.723928 & -2.534856 & 1.876374 \\ \mathrm{H} & 3.183484 & -2.232549 & 0.912796 \\ \mathrm{H} & 3.121509 & -1.704007 & 2.592787 \\ \mathrm{C} & 0.32447 & 3.321669 & -0.03591 \\ \mathrm{H} & -0.342925 & 3.250642 & -0.904385 \\ \mathrm{H} & -0.26031 & 3.76977 & 0.780294 \\ \mathrm{H} & 1.133832 & 4.015135 & -0.28567 \\ \mathrm{C} & 4.303698 & 0.431209 & 0.993403 \\ \mathrm{H} & 4.629606 & 0.224335 & 2.022062 \\ \mathrm{H} & 4.660789 & -0.404904 & 0.377675 \\ \mathrm{H} & 4.812154 & 1.338275 & 0.651103 \\ \mathrm{H} & -0.084761 & -1.171199 & 1.226497 \\ \mathrm{H} & 2.903439 & 2.615188 & 0.262778\end{array}$

TI(TFA) 3-1,2,3,5-tetramethylbenzene $\pi$-complex

$\begin{array}{llll}\text { Electronic Energy } & & -2140.632823 & \\ \text { Electronic and Zero-point Energy } & -2140.335053 & \\ \text { Enthalpy } & & -2140.299925 & \\ \text { Free Energy } & -2140.404466 & \\ \text { TI } & 0.320529 & 0.352226 & 3.524039 \\ \text { O } & 2.608125 & 0.828599 & 4.244184 \\ \text { C } & 2.450811 & 2.035345 & 3.948742 \\ \text { O } & -1.656991 & 1.657581 & 3.726083 \\ \text { C } & -2.415931 & 0.655439 & 3.63532\end{array}$




\begin{tabular}{|c|c|c|c|}
\hline O & 0.071665 & -0.229663 & 5.8586 \\
\hline C & 0.422916 & -1.405379 & 5.613922 \\
\hline C & 3.66352 & 2.956039 & 4.179179 \\
\hline C & -3.922985 & 0.950029 & 3.754471 \\
\hline C & 0.486861 & -2.42319 & 6.767631 \\
\hline O & 1.408213 & 2.525456 & 3.457133 \\
\hline O & -2.034717 & -0.516727 & 3.463229 \\
\hline $\mathrm{O}$ & 0.737402 & -1.842883 & 4.481854 \\
\hline $\mathrm{F}$ & 3.376457 & 4.237676 & 3.975586 \\
\hline $\mathrm{F}$ & 4.125644 & 2.823026 & 5.422793 \\
\hline $\mathrm{F}$ & 4.643647 & 2.609573 & 3.334924 \\
\hline$F$ & -4.288995 & 1.848713 & 2.834756 \\
\hline $\mathrm{F}$ & -4.194047 & 1.45813 & 4.959375 \\
\hline $\mathrm{F}$ & -4.664617 & -0.13986 & 3.585287 \\
\hline $\mathrm{F}$ & 0.121153 & -1.898861 & 7.93215 \\
\hline$F$ & -0.315693 & -3.459203 & 6.505617 \\
\hline $\mathrm{F}$ & 1.732992 & -2.889988 & 6.891587 \\
\hline C & 0.77099 & 1.919986 & 0.292953 \\
\hline C & 2.807669 & 0.696722 & 0.876777 \\
\hline C & 0.014336 & 0.785682 & 0.604714 \\
\hline C & 2.090184 & -0.432898 & 1.23682 \\
\hline C & 0.669441 & -0.364152 & 1.146185 \\
\hline C & 2.17573 & 1.86729 & 0.434408 \\
\hline C & -1.466302 & 0.744977 & 0.387836 \\
\hline $\mathrm{H}$ & -1.695906 & 0.821389 & -0.683466 \\
\hline $\mathrm{H}$ & -1.897344 & -0.193108 & 0.752799 \\
\hline $\mathrm{H}$ & -1.979924 & 1.58052 & 0.881007 \\
\hline C & 2.763183 & -1.678521 & 1.71055 \\
\hline $\mathrm{H}$ & 2.245467 & -2.120767 & 2.5707 \\
\hline $\mathrm{H}$ & 2.766469 & -2.43661 & 0.91546 \\
\hline $\mathrm{H}$ & 3.801434 & -1.481777 & 1.996751 \\
\hline C & 0.098644 & 3.193321 & -0.11671 \\
\hline $\mathrm{H}$ & -0.86051 & 3.01925 & -0.612331 \\
\hline $\mathrm{H}$ & -0.099551 & 3.813595 & 0.772114 \\
\hline $\mathrm{H}$ & 0.715092 & 3.791771 & -0.793992 \\
\hline $\mathrm{H}$ & 0.09345 & -1.289104 & 1.261545 \\
\hline $\mathrm{H}$ & 3.894415 & 0.685494 & 0.965456 \\
\hline C & 3.009805 & 3.07338 & 0.155016 \\
\hline $\mathrm{H}$ & 2.857463 & 3.447411 & -0.865506 \\
\hline $\mathrm{H}$ & 2.740585 & 3.896582 & 0.833004 \\
\hline $\mathrm{H}$ & 4.075746 & 2.860286 & 0.278481 \\
\hline
\end{tabular}




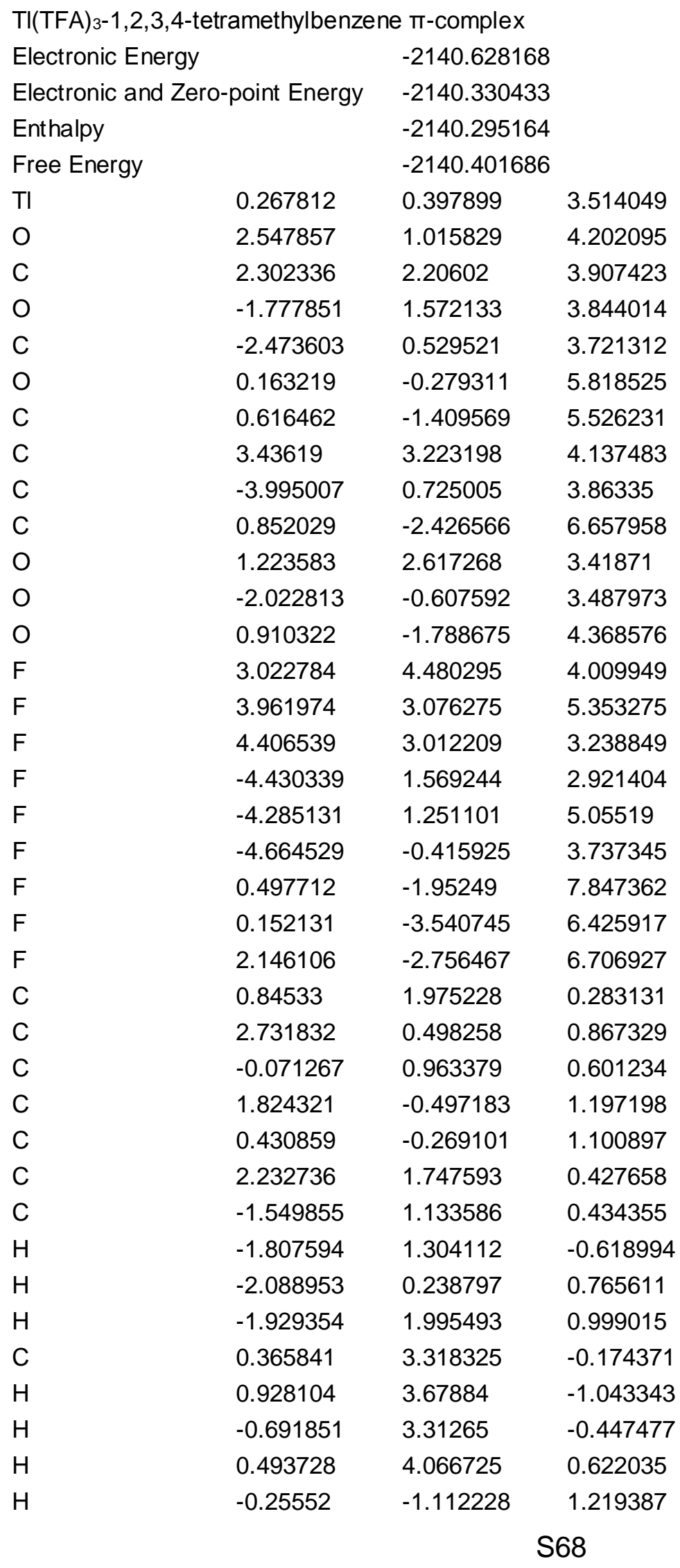




$\begin{array}{llll}\mathrm{C} & 3.173273 & 2.86895 & 0.12345 \\ \mathrm{H} & 3.155964 & 3.117108 & -0.947218 \\ \mathrm{H} & 2.878655 & 3.783697 & 0.655815 \\ \mathrm{H} & 4.207249 & 2.640741 & 0.388976 \\ \mathrm{C} & 4.199687 & 0.227558 & 1.00246 \\ \mathrm{H} & 4.731974 & 0.385401 & 0.05589 \\ \mathrm{H} & 4.671949 & 0.878407 & 1.749994 \\ \mathrm{H} & 4.371067 & -0.808069 & 1.31354 \\ \mathrm{H} & 2.176642 & -1.466504 & 1.549456\end{array}$

TI(TFA) 3 -pentamethylbenzene $\pi$-complex

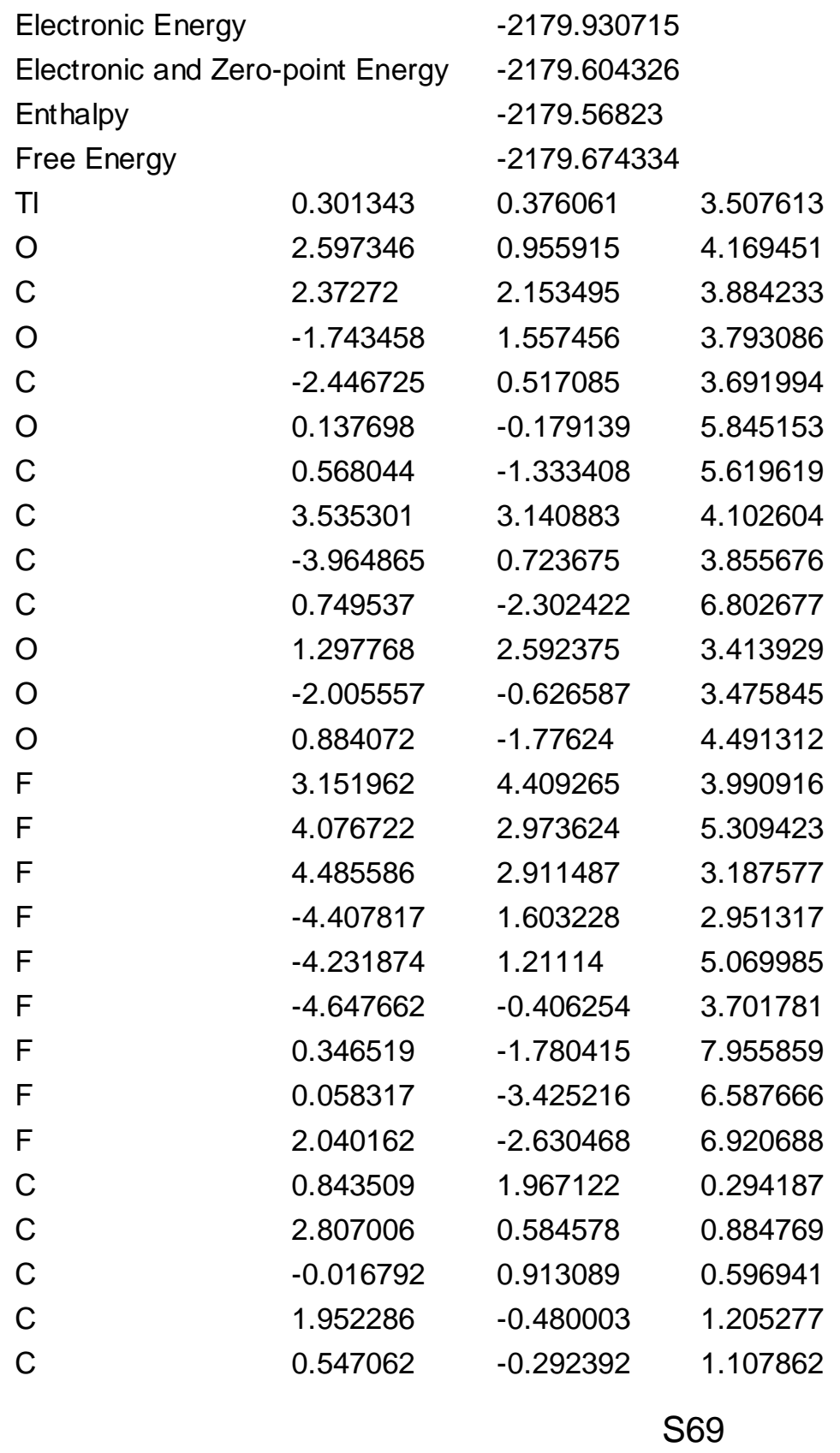




$\begin{array}{llll}\mathrm{H} & -0.098223 & -1.171597 & 1.20222 \\ \mathrm{C} & 2.246734 & 1.802352 & 0.4377 \\ \mathrm{C} & -1.500101 & 0.993663 & 0.399859 \\ \mathrm{H} & -1.743256 & 1.166596 & -0.65637 \\ \mathrm{H} & -1.990476 & 0.062409 & 0.703996 \\ \mathrm{H} & -1.946884 & 1.819781 & 0.968491 \\ \mathrm{C} & 2.483832 & -1.800378 & 1.657613 \\ \mathrm{H} & 1.678161 & -2.521642 & 1.827436 \\ \mathrm{H} & 3.166594 & -2.223364 & 0.908929 \\ \mathrm{H} & 3.059058 & -1.70809 & 2.589908 \\ \mathrm{C} & 3.0958 & 2.991831 & 0.119532 \\ \mathrm{H} & 2.886279 & 3.80932 & 0.825625 \\ \mathrm{H} & 4.166884 & 2.79365 & 0.144683 \\ \mathrm{H} & 2.860823 & 3.385915 & -0.876924 \\ \mathrm{C} & 0.302316 & 3.291168 & -0.150662 \\ \mathrm{H} & 0.684908 & 3.581751 & -1.137502 \\ \mathrm{H} & -0.788071 & 3.297138 & -0.208717 \\ \mathrm{H} & 0.597714 & 4.084234 & 0.550909 \\ \mathrm{C} & 4.281241 & 0.35591 & 1.047389 \\ \mathrm{H} & 4.510783 & 0.013186 & 2.065176 \\ \mathrm{H} & 4.632913 & -0.431116 & 0.366393 \\ \mathrm{H} & 4.890746 & 1.239921 & 0.858862\end{array}$

\section{References}

1. Faggiani, R.; Brown, I. D. Acta Crystallogr., Sect. B 1978, B34, 2845-2846.

2. W. Lau, J. K. Kochi, J. Am. Chem. Soc. 1986, 108, 6720-6732.

3. Cramer, C. J., "Charge Distribution and Spectroscopic Properties" in Essentials of computational chemistry: Theories and models. 2nd ed.; J. Wiley: Hoboken, NJ, 2004.

4. Anderson, G. M.; Cameron, I.; Murphy, J. A.; Tuttle, T., RSC Adv. 2016, 6, 11335-11343.

5. Guo, Y.; Wang, W.; Shao, R.; Yin, S., Comp. Theor. Chem. 2015, 1057, 67-73.

6. E. C. Taylor, F. Kienzle, R. L. Robey, A. McKillop, J. D. Hunt, J. Am. Chem. Soc. 1971, 93, 4845-4850.

7. G. A, Olah, I. Hashimoto, H. C. Lin, Proc. Natl. Acad. Sci. U.S.A. 1977, 74, 4121. 